Portland State University

PDXScholar

Spring 6-2-2014

\title{
The Impact of School-Level Factors on Minority Students' Performance in AP Calculus
}

Phillip Bruce Pearson

Portland State University

Follow this and additional works at: https://pdxscholar.library.pdx.edu/open_access_etds

Part of the Science and Mathematics Education Commons Let us know how access to this document benefits you.

\section{Recommended Citation}

Pearson, Phillip Bruce, "The Impact of School-Level Factors on Minority Students' Performance in AP Calculus" (2014). Dissertations and Theses. Paper 1849.

https://doi.org/10.15760/etd.1848

This Dissertation is brought to you for free and open access. It has been accepted for inclusion in Dissertations and Theses by an authorized administrator of PDXScholar. Please contact us if we can make this document more accessible: pdxscholar@pdx.edu. 
The Impact of School-Level Factors on Minority Students'

Performance in AP Calculus

by

Phillip Bruce Pearson

A dissertation submitted in partial fulfillment of the requirements for the degree of

Doctor of Education

in

Educational Leadership: Administration

Dissertation Committee:

Dilafruz Williams, Chair

Samuel Henry

Swapna Mukhopadhyay

Joel Steele

Portland State University

2014 
(C) 2014 Phillip Bruce Pearson 


\section{Abstract}

In recent years, Science, Technology, Engineering, and Technology (STEM) talent pool has re-emerged as a national priority. Certain racial and ethnic groups are dramatically underrepresented in STEM careers and STEM educational programs, an especially serious concern given demographic transitions underway in the United States. The College Board's Advanced Placement (AP) Calculus program provides one way in which students can gain exposure to college-level mathematics while still in high school. This study analyzed factors that contribute to the success of minority students in AP Calculus using a large, longitudinal (2007-2012), geographically distributed dataset which included important school-level variables and AP scores for 10 urban school districts. Descriptive statistics show that AP success in general and minority success in AP Calculus specifically are unevenly distributed across the dataset. A very small number of schools and school districts account for the majority of the production of passing scores on AP exams. Results from multivariate regression and multi-level growth modeling demonstrate that school size and academic emphasis on a school level constitute important predictors of success for Black and Hispanic students in AP Calculus. The very narrow distribution of AP success across schools and school districts suggests that a specific set of school-level policies and practices are likely to be highly effective in leveraging these two predictors. 


\section{Acknowledgements}

This project would have died a quiet death if not for the efforts of two brilliant and compassionate social scientists. Dr. Karen Noordhoff was present at the beginning and intervened at a key moment to preserve progress and momentum. Dr. Dilafruz Williams stepped in 18 months ago. Her zest, her passion, and her assertiveness saved this dissertation from extinction. These two taught me what a difference dedicated educational researchers can make in the world. My debt to them is wider than words can describe. The support of my doctoral committee-Dr. Swapna Mukhopadhyay, Dr. Samuel Henry, and Dr. Joel Steele--has also been critical. Dr. Steele and Dr. Masami Nishishiba brought critical expertise to bear on the analysis. I am grateful to them both. Important support also came from my friend and boss Dr. Randy Trani and many of my colleagues in Corbett School District. Primarily, though, it has been my wife Angela, who, for some reason, has believed in me since the moment we met a long time ago. She is the reason I'm still standing. Our daughter Elie has also been a constant ray of sunshine. This morning she sat in my lap reading this document. "There are a lot of long words in this," she said, "Are there supposed to be so many long words?" I hope so, and I hope some of them make a difference. Whatever difference they might make I owe to these people I've named and to many, many others. Thank you all. 


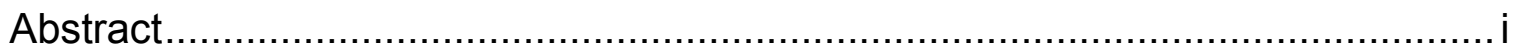

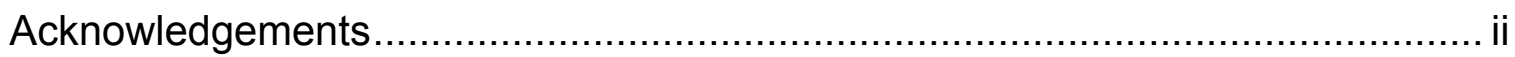

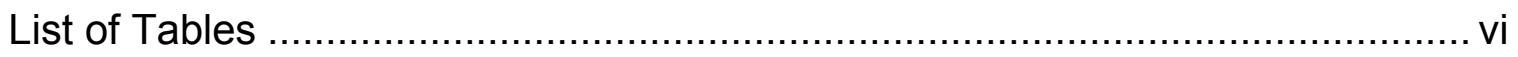

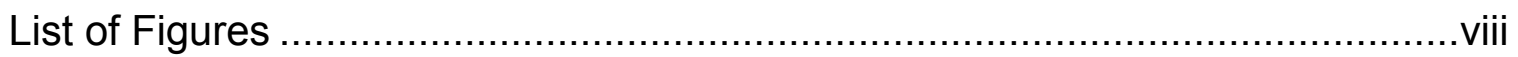

Chapter

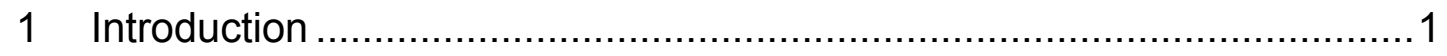

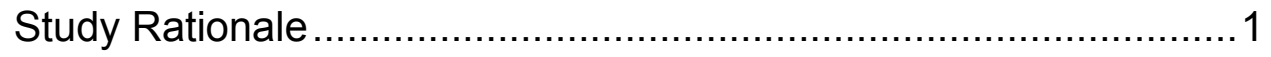

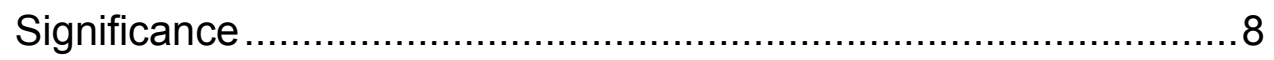

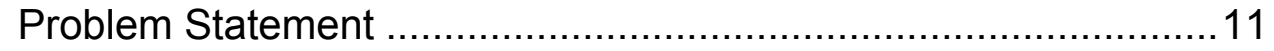

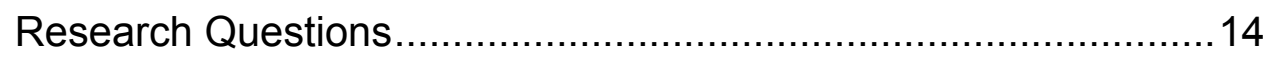

Definition of Key Terms .......................................................... 15

Definition of Acronyms …..................................................... 17

2 Review of Related Literature ........................................................ 18

Structure of Literature Review................................................18

F1: Historical Social Contextual Factors …................................22

The Formation of Prejudice and Out Groups.......................23

Voluntary Versus Involuntary Immigrants ............................26

Oppositional Culture and the Burden of Acting White ...........29

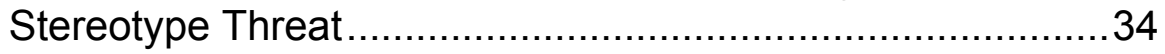

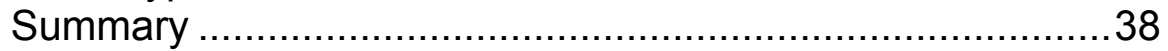


F2: Parental Expectations and the Cultural Capital Explanation....41

The Effect of Parental Expectations on Achievement ...........41

The Cultural Capital Explanation ..........................................44

Summary ................................................................ 4

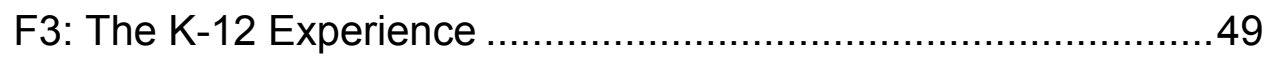

Educational and Economic Indicators .................................50

F3-1: Funding Disparities ...............................................52

F3-2: Disparities in Teacher Qualifications and Credentials..56

F3-3: Tracking and Differential Enrollments in College-

Preparatory Courses ......................................................60

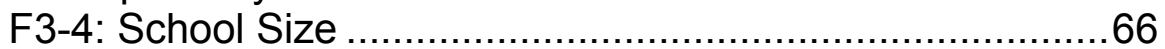

F3-5: School Choice and Governance ...............................69

F3-6: Academic Momentum ......................................... 73

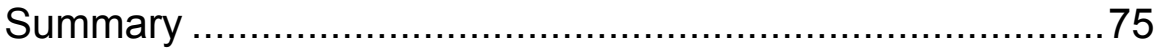

A Spotlight on AP Calculus Achievement ........................................

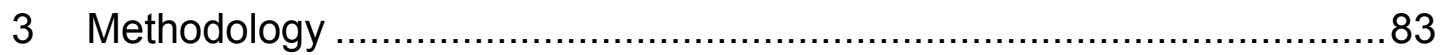

Selection of Sample and Data Collection ....................................83

Data Processing and Data Equivalence.......................................85

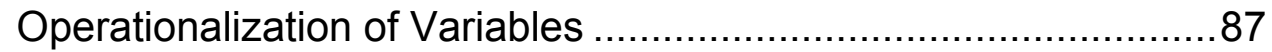

School-Aggregated SES and Ethnic Composition .......................90

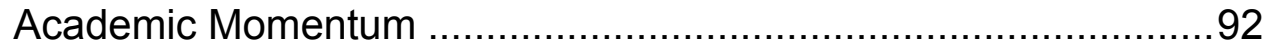

Computation of Derived Variables ...........................................93

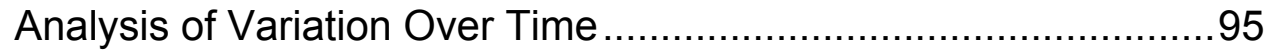

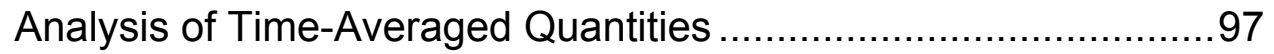

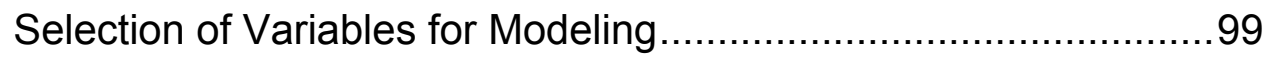

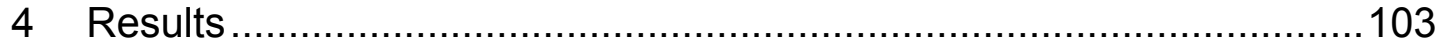

Descriptive Statistics ...........................................................103 


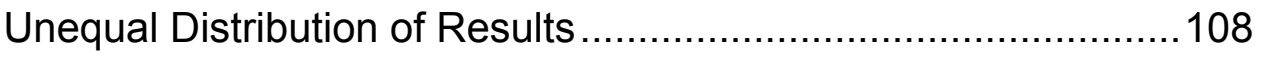

Mixed Models to Analyze Change Over Time ...........................111

Unconditional Individual Growth Model .............................112

Fully Specified Individual Growth Model............................113

Partially Specific Individual Growth Model.........................116

Time Averaged Regressions .................................................119

Predictors of Minority AP Calculus Production ...................120

Isolation of Variables in Data Set One ....................................134

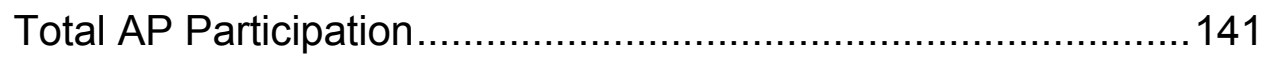

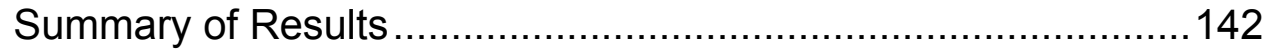

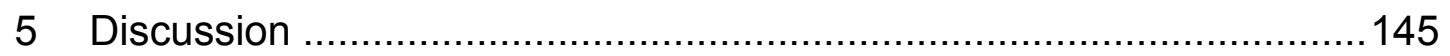

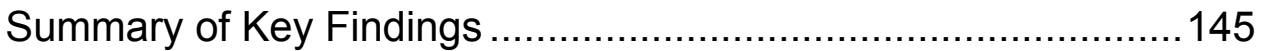

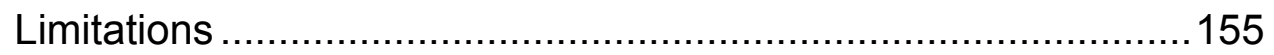

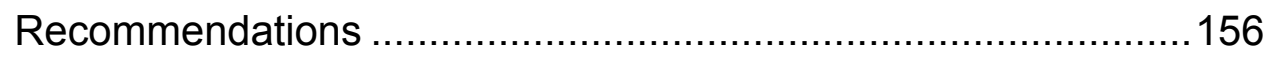

Opportunities for Future Research ..........................................160

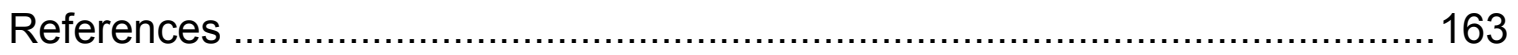

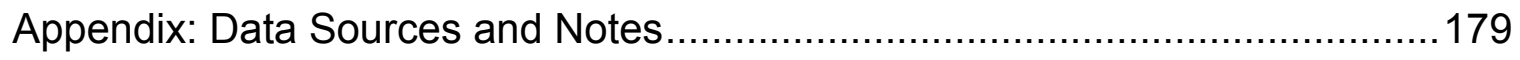


List of Tables

Table

Page

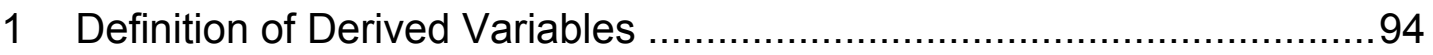

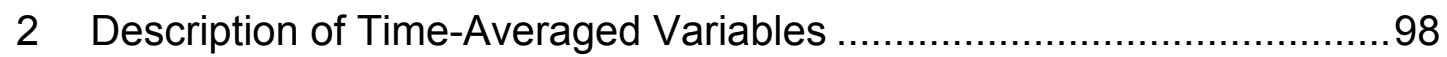

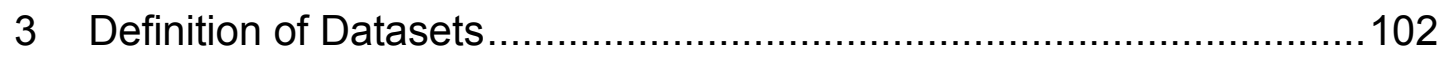

4 Correlations Between Time-Averaged Variables ................................105

5 Descriptive Statistics of Time-Averaged Variables ............................107

6 Comparison Between Top Producers of Minority AP Calculus and the Total Population ............................................................. 111

7 Comparison of Results for Unconditional Growth Models ...................112

8 Variances and Model Fit, Fully-Specified Growth Models ...................114

9 Estimates of Coefficients, Fully-Specified Growth Models ..................114

10 Interaction Effects for Fully-Specified Growth Models .........................115

11 Results of Partially-Specified Growth Models ..................................116

12 Comparison of Interaction Effects for Partially-Specified Growth

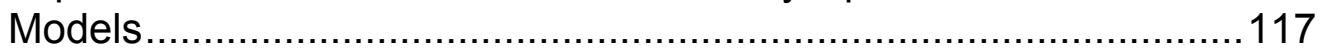

13 Comparison of Model Outputs, Partially-Specified Growth Models ......118

14 Results of Fully-Specified Models, Time-Averaged Regressions .........120

15 Results of Regression for Partially-Specified Models, Time-

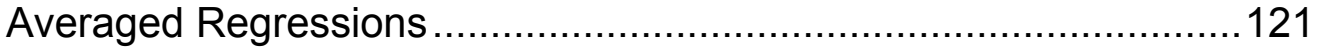

16 Governing Equations for Fully-Specified Models, Datasets One Through Four

17 Governing Equations for Partially-Specified Models, Datasets One Through Four.

18 Comparison of Results for Supplemental Analysis, Dataset One. 135 
19 Comparison of Fully- and Partially-Specified Models, Total NonCalculus Production as Outcome Variable

20 Governing Equations for Fully- and Partially-Specified Models, Non-Calculus and Dependent. 


\section{List of Figures}

Figure

1 Factors Impacting Achievement of Minority Students in STEM 19

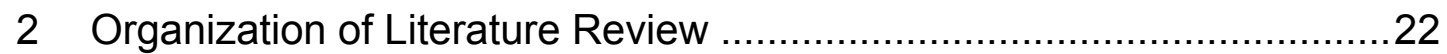

3 AP Calculus Score Distributions for Selected Groups, $2011 \ldots \ldots \ldots \ldots \ldots . . . .79$

4 AP Calculus Score Distributions for Minority Students, Selected States....

5 Proportion of AP Calculus Test Takers Scoring 3 or Better, Minority Students, Selected States

6 Comparison of Minority AP Production Rates to Normal Distribution ...107

7 Comparison of Total AP Production Rate to Normal Distribution .........108

8 Histogram of Passing Scores, Total and Minority Populations .............109

9 Cumulative Histogram of Passing Scores, Minority Students, AP

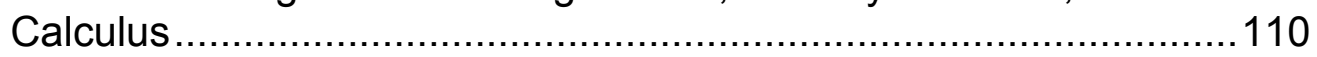

10 Histogram of Unstandardized Residuals, Dataset Four......................125

11 Q-Q Plot of Unstandardized Residuals, Dataset Four ........................126

12 Histogram of Unstandardized Residuals, Dataset Three.....................127

13 Q-Q Plot of Unstandardized Residuals, Dataset Three ......................128

14 Histogram of Unstandardized Residuals, Dataset Two .......................129

15 Q-Q Plot of Unstandardized Residuals, Dataset Two .........................130

16 Histogram of Unstandardized Residuals, Dataset One ......................131

17 Q-Q Plot of Unstandardized Residuals, Dataset One .........................132

18 Histogram of Unstandardized Residuals for Partially-Specified Model, Non-Calculus AP Production 
19 Q-Q Plot of Unstandardized Residuals for Partially-Specified Model, Non-Calculus AP Production ...........................................141 


\section{CHAPTER I \\ INTRODUCTION \\ Study Rationale}

Science, Technology, Engineering, and Mathematics (STEM) education first entered the national spotlight in 1957 with the launch of sputnik and the inauguration of the space race. In 1983, STEM education-and the state of the education in general-received renewed and intense scrutiny with the publication of A Nation at Risk (United States Department of Education, 1983). This highly influential report suggested that American leadership in science and technology had largely eroded and that, according to a wide range of indicators, education in the United States had slumped into international mediocrity. The report specifically identified deficits in STEM course taking among high school students and shortages in terms of teacher pay, teacher credentials, and the size of the available labor pool in STEM education (United States Department of Education, 1983). The report, introduced by President Ronald Regan on April 26, 1983, received wide attention in the popular media and launched waves of school reform aimed at school accountability and standardization (Graham, 2013).

In recent years, STEM education has reentered the national spotlight and has, once again, become a national priority. In his 2013 budget, President Obama called for a $2.6 \%$ increase in spending over 2012 levels to support STEM education specifically. This increase, amounting to $\$ 126$ million, signals a 
renewed commitment to "improve the quality of science, technology, engineering, and mathematics (STEM) education at all levels" (United States Office of Science and Technology Policy, 2012, p. 1). In December of 2012, the President called on educators to produce 1 million more STEM graduates in the next 10 years and formally designated that target as a Cross Agency Priority goal (Larson, 2012). On February 13, 2013, the STEM Jobs Act was introduced to the House of Representatives and referred to committee for further consideration. The bill, which has attracted support from the Semiconductor Industry Association and The American Institute of Aeronautics and Astronautics, would provide green cards to foreign students who earn advanced degrees in STEM fields from institutions in the United States (Conyers, 2012; STEM Jobs Act). These legislative efforts are in keeping with sentiments echoed by business leaders who suggest that "it is clear that to benefit our economy and society, our national priority should be on encouraging more students to study STEM" (Adkins, 2012, para. 3).

A renewed interest in STEM education is also evident on the state level. In 2011, Oregon business and educational leaders released a draft of the "Oregon STEM Initiative" designed to define specific goals, metrics, and methods for improving STEM education throughout the state (Oregon Department of Education, 2011b). The initiative envisions Regional STEM Centers which will draw together a range of educators and educators from around the state. The goals of these centers closely follow STEM goals established at the national level 
that Include the following: improve student performance on STEM content, increase student interest in STEM fields, and improve teaching of STEM content (Oregon Department of Education, 2011b).

While the number of bachelor's degrees in science has increased steadily over the past decade (National Science Board, 2012) and while "in most broad aspects of S\&T [science and technology] activities, the United States continues to maintain a position of leadership" (National Science Board, 2012, section O, p. 3), troubling signs are apparent. In 2012, there were 7 million STEM jobs available in the United States. By 2018, this number is expected to grow to nearly 9 million (My College Options and STEMconnector, 2012). As the number of opportunities in STEM increases, "STEM employers throughout the U.S. report shortages of skilled workers" (Wang \& Degol, 2013, p. 305). International comparisons of American students in STEM disciplines is, in many cases, disappointing. For instance, 15-year-olds in the United States continue to perform below the international average on the PISA (Program for International Student Assessment) exam for mathematical literacy. Overall, the United States ranks $18^{\text {th }}$ out of the 33 member nations of the Organization for Economic Cooperation and Development. Additionally, "while a majority of ninth graders reached proficiency in low-level algebra skills, few mastered higher level skills" (National Science Board, 2012, section 1, p. 34).

Perhaps one of the most pernicious and persistent characteristics of STEM education in the United States is the oft-noted achievement gap. Native 
American, Hispanic, and Black students continue to be dramatically underrepresented in STEM, both in terms of achievement and participation. In 2000 , approximately $6 \%$ of all 24 year olds held a bachelor's degree in a STEM field compared to just under $5 \%$ of those 24 year olds who were Black, NativeAmerican, or Hispanic. During that same year, 12-15\% of all bachelor's degrees and $2-3 \%$ of all doctoral degrees in STEM fields were awarded to underrepresented minorities as compared to a total rate of representation in the college-aged population of approximately $30 \%$ (National Science Board, 2004). Students from these same ethnic groups also posted lower scores than their White classmates at all grade levels on standardized assessments of science and mathematics achievement (S. Lewis, Simon, Renata, Horwitz, \& Casserly, 2010, 2011; National Science Board, 2004).

These results are especially troubling given current demographic trends in the United States. In 2012, Blacks, Native Americans, and Hispanics represented $13 \%, 1.2 \%$, and $17 \%$ of the total population respectively. By 2060 , those proportions are expected to reach 15\%, 1.5\%, and 31\%. In 2043, the United States is expected to become a "minority-majority" nation for the first time meaning that, while non-Hispanic Whites will still be the largest single group, no ethnic group will constitute a majority (United States Census Bureau, 2012). Without a concurrent shift in minority participation and achievement in STEM, the United States will be increasingly hard pressed to maintain its position of leadership in science and engineering over the coming decades. 
One key predictor of achievement in STEM at both the secondary and postsecondary levels is a student's pattern of course taking in the sciences and mathematics. Indeed, "the accumulated evidence concerning the importance of science and mathematics coursework in high school is overwhelming" (Tyson, Lee, Borman, \& Hanson, 2007, p. 244). Completing challenging STEM coursework has been demonstrated to improve SAT scores (Riegle-Crumb, 2006) and ACT math scores (American College Testing, 2005). Patterns of rigorous course taking have also been positively associated with post-high school earnings (Rose \& Betts, 2004) and with earning a bachelor's degree regardless of major (Adelman, 1999). The highest level of math completed has specifically been associated with finishing a bachelor's degree and enrolling in a 2- or 4-year postsecondary institution (Adelman, 1999; Engberg \& Wolniak, 2010). Adelman (2006) reported that

however complex a student's attendance pattern, the principal story leading to degrees is that of content... The academic intensity of a student's high school curriculum still counts more than anything else in precollegiate history in providing momentum towards completing a bachelor's degree. (Executive Summary, p. 17)

A student's journey through the STEM educational system has variously been described as a circuit, a pathway, or a pipeline. Whatever the metaphor, "the trends described here ... appear to support a sequential sorting model, with more students being sorted out of the pipeline with age and those who remain being less diverse" (Jacobs, 2005, p. 90). In their examination of the National Longitudinal Study of the High School class of 1972 and the High School and 
Beyond dataset of 1982, Hilton and Lee (1988) determined that the greatest point of attrition from STEM disciplines was between high school graduation and college. Interestingly, Black and Hispanic students who complete advanced level course work in science and mathematics are more likely than White students to complete a STEM bachelor's degree (Tyson et al., 2007), underscoring again the importance of the high school academic experience. Because rates of early school leaving for Black, Hispanic, and Native-American students are considerably higher than for White students (Chapman, Laird, \& Kewel Ramani, 2010), factors which encourage school persistence to graduation can also be considered to be relevant to equity in STEM. This last point is also important in a more general sense because levels of academic achievement are closely associated with a wide variety of quality of life indicators, including likelihood of being incarcerated (Harlow, 2003), personal income (United States Census Bureau, 2012), and unemployment trends (United States Bureau of Labor Statistics, 2011).

It is clear that providing for greater equity in STEM education in the future will rest in no small degree on "the need to better understand the secondarypostsecondary nexus and the structures and organizational norms that are most conducive in enabling students to make the journey from high school to college" (Engberg \& Wolniak, 2010, p. 2). The high school context is critical, and the extent to which that context enables success in rigorous, high-level coursework 
in mathematics and science plays a key role in a student's progress along the STEM pathway.

The College Board's Advanced Placement (AP) program provides one mechanism through which students can encounter collegiate-level, advanced mathematics while still in high school. A number of investigators have shown that students who take AP courses in high school do better in college. In one example, students who took AP courses were matched with students who did not take AP courses but who had similar American College Test (ACT) or Scholastic Aptitude Tests (SAT) scores and class rankings. In all nine subject areas examined, AP students outperformed non-AP students in college outcome measures during their first 3 years of college (Murphy \& Dodd, 2009). In a study matching AP students with non-AP students with similar SAT rankings and socioeconomic status (SES), AP students significantly outperformed non-AP students in terms of college GPA (grade point average) and graduation rates (Hargrove, Godin, \& Dodd, 2008). These results held regardless of how well the students performed on the end of year exam. After controlling for student characteristics and prior achievement, Sadler and Tai (2007) found that taking AP Physics, Chemistry, and Biology provided students with statisticallysignificant advantages in those courses at the postsecondary level. Similar results were obtained for AP Calculus students (Mattern, Shaw, \& Xiong, 2009), although, after controlling for previous academic performance, only students who scored 3,4 , or 5 (on a 5-point scale) obtained statistically significant advantages. 
Black and Hispanic students have historically under-participated and under-performed in AP Calculus relative to their White classmates. In 2011, Black students took $5.5 \%$ of all AP Calculus exams administered in the United States and obtained an average score of 1.79 while Hispanic students took approximately $12 \%$ of all AP Calculus exams and obtained an average score of 2.0. White students, in contrast, took $62 \%$ of all AP Calculus exams and obtained an average score of 2.94 (The College Board, 2011). Students who either under participate or under achieve in advanced mathematics in high school are less likely to succeed in postsecondary STEM education. The proposed investigation seeks to illuminate those school-level factors which positively impact the underrepresentation-both in terms of participation and in terms of average scores-of minority students in AP Calculus.

\section{Significance}

By 2035, more than 130 million Americans will be of Black or Hispanic heritage. By 2060, non-Hispanic Whites will no longer represent a majority of the population. In 2009 approximately $17 \%$ and all Hispanic students and $10 \%$ of all Black students aged 16-24 dropped out of school in 2009 (Chapman et al., 2010). In 2000 , approximately 9,000 and 8,000 science and engineering master's degrees were awarded to underrepresented minority students and Asian students respectively. In contrast, White students accounted for approximately 50,000 science and engineering master's degrees (National Science Board, 2004). In 2001, fewer than 1,500 doctoral degrees in science and engineering 
degrees were awarded to Black and Hispanic students or less than one tenth the number of science and engineering doctoral degrees awarded to White students (National Science Board, 2004).

Increasing minority participation in STEM disciplines at the postsecondary level is important for at least three reasons. First, levels of educational attainment in general are closely correlated with a wide variety of quality of life indicators. Second, workers in STEM disciplines generally enjoy higher salaries than workers in other industries. This salary differential, in turn, can translate into increased economic opportunities for minority students and their families into successive generations. The average annual salary for all STEM occupations was $\$ 77,880$ in 2009 , compared to mean annual wage in the United States of $\$ 43,460$. Only four of the 97 STEM occupations identified by the United States Bureau of Labor Statistics had average wages that were below the average wage in the United States. All of the managerial STEM occupations had yearly wages in excess of $\$ 100,000$. Additionally, STEM occupations account for more than half of all jobs in a number of critical industries, including computer manufacturing, software design, architecture, and scientific research and development (Cover, Jones, \& Watson, 2011). Unemployment rates for STEM occupations also compare favorably against other occupations. In June of 2012, U.S. News and World Report reported that, for non-STEM occupations, there are 3.6 unemployed workers for every available job. In contrast, for every 
unemployed STEM worker there are two STEM jobs currently unfilled (Engler, 2012).

Third, the United States pays a heavy economic price when a sizable fraction of the total talent pool exits STEM disciplines before those individuals obtain advanced degrees and begin to contribute economically and in terms of innovation. According to a 2004 NSF estimate, knowledge-intensive service industries in the United States generated between $\$ 3.5$ and $\$ 4.0$ billion in global income. During that same period, approximately 400,000 undergraduate degrees in science and engineering were awarded, about 64,000 of which were awarded to underrepresented minorities (National Science Board, 2004). Using an equity index developed by J. Lewis, Menzies, Najera, and Page (2009) as a rough guide, that output represented an under production of bachelor's degrees in STEM fields to minority students of about 60,000 degrees, or about $15 \%$ of the total output of bachelor's degrees. Given the global economic impact of knowledge-intensive service industries, it is reasonable to estimate that this $15 \%$ rate of under production represents a loss of many hundreds of millions of dollars to the United States economy. Writing for the U.S. News and World Report, Engler (2012) suggested that "for the United States to remain the global innovation leader, we must make the most of all of the potential STEM talent this country has to offer" (p. 1).

In summary, while STEM education has become a national priority and while minority students represent a large and growing proportion of the total 
STEM talent pool, there exists a substantial deficit in the production of STEM postsecondary degrees for minority populations. While all points along the socalled K-12 STEM "pipeline" are critical to the overall academic success of minority students in STEM, a student's high school experience is of particular importance. Elucidating those factors which impact minority students' success in STEM at the high school level therefore represents an important contribution to the national goal of increasing the production of STEM degrees. Finally, and perhaps most importantly, advancing the cause of equity in the "STEM educational circuit is a moral and ethical imperative" (Museus, Palmer, \& Davis, 2011, p. 5).

\section{Problem Statement}

A number of factors relating to a student's high school academic experience have been shown to be important predictors of a student's likelihood of obtaining a postsecondary degree in general and that student's likelihood of completing a STEM major specifically. Students from some minority groups dramatically underachieve compared to White test takers on the AP Calculus exam both in terms of rates of participation and average scores. During 2011, for example, the average exam score for White students was 2.94 on a 5-point scale, indicating that White students, on average, tended to achieve at levels that were considered "college proficient," or nearly so. In contrast, Black students averaged a score of 1.79 and Hispanic students averaged a score of approximately 2.0 (The College Board, 2011). This score deficit of one point 
represents a much lower "pass" rate for Black and Hispanic students. Students who identified themselves as being of Asian descent outperformed all groups, reporting and average score of 3.11 on the AP Calculus exam.

The deficits described above are especially vexing because math achievement is largely a cumulative process. Those minority students who take the AP Calculus exam in high school, have, of necessity, demonstrated achievement in lower levels of math during their earlier grades. Students who are not highly math motivated or those who have been tracked into lower, nonacademic tracks will most often not take AP Calculus in high school because they likely lack the prerequisite coursework. In addition, in most cases students must be invited into AP courses by teachers who view those students to be capable of doing well on the AP exam. AP Calculus students, therefore, represent a non-representative pool of students who have been selected according to their previous achievement and perceived potential.

The strength of students' high school mathematics preparation is particularly important in terms of success in postsecondary success in STEM. Identifying school-level factors which promote success in high school mathematics is therefore of critical importance, particularly where Black, Native American, and Hispanic students are concerned. The participation of these groups in AP Calculus constitutes one important representation of high school achievement in mathematics. The current investigation seeks to identify schoollevel factors which contribute to the success of Black and Hispanic students in 
AP Calculus and, by extension, to their likely success in postsecondary STEM educational programs.

Although the experience of Native American students is certainly of vital concern, these students are excluded from this investigation due to concerns over statistical significance of results and the maintenance of individual confidentiality. In 2011, 1,026 AP Calculus test takers identified themselves as American Indian, a number which has grown slowly and steadily over the past decade (The College Board, 2011). While this is an encouraging trend in terms of participation, these rates represent less than $0.5 \%$ of the total volume of AP Calculus exams administered nationally. Very small sample sizes create concerns over the significance and generalizability of results. In addition, on a school level sample sizes could become so small as to make individual students identifiable.

Asian students are similarly excluded because these students, as a group, exceed the average performance for all students on AP Calculus. While fruitful investigation could certainly be conducted into the experiences of these students, the current investigation seeks to identify school-level factors which ameliorate weaknesses within the STEM educational pipeline. Those students who identify themselves as Asian outperform the national average in AP Calculus while Black and Hispanic students underperform the national average in AP Calculus. Black and Hispanic students, therefore, constitute salient groups for this investigation and Asian students fall outside of the scope of these analyses. However, it is 
important to note that the over generalization of Asian students as a group has the potential to exacerbate the very inequities this investigation and others like it seek to ameliorate. Creating a single category for all Asian students and assuming all students within that group post strong academic results serves to hide important variations. The school experiences of subgroups within that population identified as Asian represent a critical area for future investigation.

\section{Research Questions}

The success of minority students in AP Calculus is conceptualized as a growth process, and many of the school-level predictors identified in proceeding sections are time-variant. It is further hypothesized that time-averaged and timevariable school-level predictors have a significant impact on the growth trajectories of the success of minority students in AP Calculus. Accordingly, the following research questions have been identified:

1. What school-level factors relate to a larger rate of growth (larger slope) in the participation by minority students on AP Calculus?

2. What school-level factors relate to a larger rate of growth (larger slope) in the achievement by minority students on AP Calculus?

The literature review in the next chapter elucidates and describes those variables which might reasonably be expected to impact this analysis. Stated briefly, the following school-level factors are expected to be important: (a) School funding and resources, (b) Teacher qualifications and credentials, (c) School size, (d) Participation in college-preparatory course work, (e) School choice and governance, (f) Demographic makeup of the school's student body, and (g) "Academic momentum" defined as changes in rates of proficiency on state 
assessments and changes in rates of production of passing scores on AP exams. Derived variables are more fully defined in chapter 3. To allow for numerical analysis, each of these factors will be fully operationalized in the methodology section. The following hypotheses are identified based on the body of results described in the literature review:

1. School size will be positively related to the growth the participation of minority students in AP Calculus but negatively related to the growth in achievement of minority students in AP Calculus.

2. Variables relating to school resources (e.g., teacher qualifications, student-teacher ratio, school-level funding) will be positively related to the growth of both the participation and achievement of minority students in AP Calculus.

3. Academic momentum will be positively related to the growth of both the participation and achievement of minority students in AP Calculus.

\section{Definition of Key Terms}

Adopting the approach of Museus et al. (2011), the following race-related terms are used throughout this dissertation:

- Black: This term is used to refer to persons with origins in any of the Black racial groups of Africa, the Caribbean, the Americas, or other regions. Because students not of African descent might identify themselves as Black, the term African American is not used. However, the term African American is commonly utilized throughout the literature. Where the term African American appears in quoted material, it is retained. In other instances, the term Black is used and assumed to be interchangeable with the term African American. The College Board utilizes the term Black in the same way and does not differentiate between the various populations listed above.

- Hispanic: This term is used to refer to persons with origins in Mexico, Central or South America, Puerto Rico, Cuba, or other Spanish communities. The College Board disaggregates Hispanic students into Mexican Americans, Puerto Ricans, and other Hispanics. For this analysis, all the groups are aggregated into a single categoryHispanics. 
- White: This term is used to refer to persons with origins in Northern Europe, North Africa, or the Middle East.

- Native American: This group is not included in the analysis for the reasons described above. The College Board uses the term American Indian and does not differentiate between the various populations which fit inside this category.

- Asian American: The College Board utilizes the term Asian while Museus et al. (2011) utilized the term Asian American and Pacific Islander to refer to persons with origins in "East Asia, Southeast Asia, the Indian subcontinent, and the Pacific Islands" (p. 7). This level of categorization neglects important variation within the population it represents. For instance, those students who identify themselves as Asian on the AP Calculus exam consistently outperform those who identify themselves as White. However, Pacific Islanders in Washington state are statistically more likely to be from poor, single family homes, have higher rates of absenteeism and early school leaving, and fare more poorly on standardized measures of math and science achievement (Washington State Commission on Asian Pacific American Affairs, 2009). Given these results, it is reasonable to suggest that significant and systematic variation in AP Calculus scores might exist within the Asian population and that this variation represents an important area for research. Unfortunately, The College Board does not collect data which are disaggregated to this extent, rendering the analysis of populations with this large and diverse group impossible. Because students who identify themselves as Asian on the AP Calculus exam as a group consistently outperform White students and because this investigation seeks to identify school level factors which contribute to the success of underrepresented populations in AP Calculus, Asian students are not included in this analysis.

Any categorization of race neglects variation with the groups identified. In effect, categorization of this sort helps to mask the diversity that is in fact the key component of interest. Furthermore, many individuals are of mixed-racial heritage and race is a self-reported descriptor. These factors represent important but unavoidable limitations and necessitate a cautious interpretation of results. 


\section{Definition of Acronyms}

- STEM: Science, Technology, Engineering, and Mathematics

- AP: Advanced Placement, The College Board's program of collegepreparatory curriculum.

- SES: Socioeconomic Status

- SAT: Scholastic Aptitude Test

- OSSI: Oregon Small Schools Initiative 


\section{CHAPTER 2}

\section{REVIEW OF RELATED LITERATURE \\ Structure of Literature Review}

This literature review aims to establish those school level factors which have been identified as having particular impact on minority achievement in STEM disciplines and in mathematics specifically. Museus et al. (2011) offered an important conceptual model which describes the interactions of multiple factors throughout a student's STEM career. These factors are grouped into seven principal areas: parental expectations, financial influences, $\mathrm{K}-12$ experiences, $\mathrm{K}-12$ outcomes, college influences, college outcomes, and STEMspecific opportunities and programs. Each of these areas are further broken into multiple components. In this model, the $\mathrm{K}-12$ experience and $\mathrm{K}-12$ outcomes are viewed as having both a direct impact on STEM college outcomes and an indirect impact through the mediating variable of financial influences. The K-12 experience and $\mathrm{K}-12$ outcomes are, in turn, directly impacted by parental influences and expectations and STEM-specific opportunities and programs. K12 outcomes are also independently influenced by K-12 experiences. The major components of the Museus model are depicted in Figure 1. 


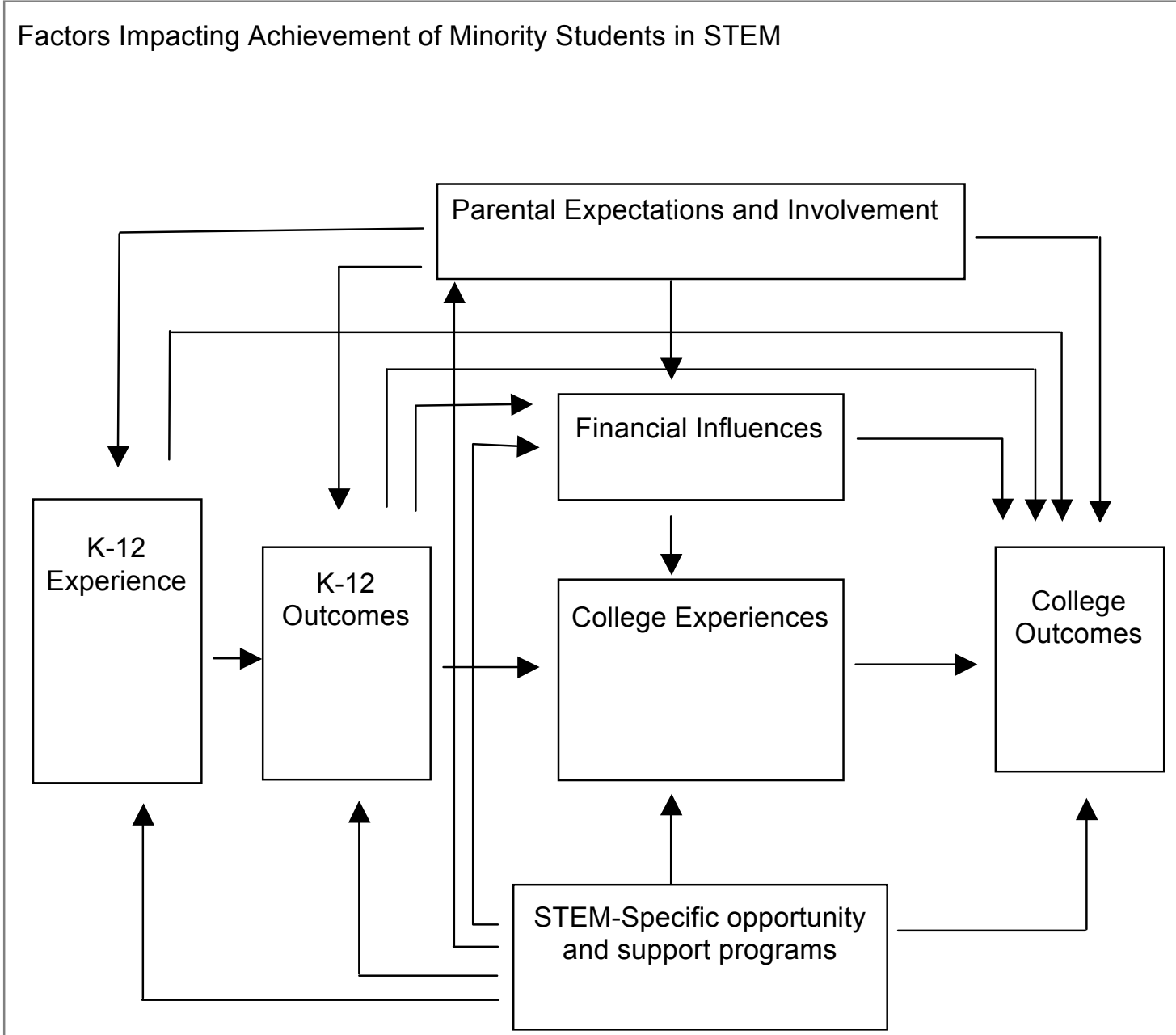

Schematic representation of major factors impacting the achievement of minority students in postsecondary STEM educational experiences, from Museus et al. (2011).

Figure 1. Factors Impacting achievement of minority students in STEM.

Within each of these areas there exists a significant potential for effective and useful investigative research. However, the focus of this investigation is on those school level factors in place in American high schools which have been demonstrated to impact the achievement of minority students in math. Attention in this literature review is therefore restricted to those variables in Museus et al.'s 
framework relating to the $\mathrm{K}-12$ grades experience generally and the $9-12$ grades experience specifically. Participation in AP Calculus is identified as the single K12 outcome of interest because it is judged to constitute one important representation of high school mathematics achievement.

While broadly inclusive, the Museus model (called Racial and Ethnic Minorities in STEM, or REM STEM) does not include some of those variables listed in their earlier discussion of "contributors to the insufficient preparation of minority students in STEM" (Museus et al., 2011, p. 29). Specifically, while funding inequities, differential teacher expectations and qualifications, and tracking are included within REM STEM, oppositional culture, stereotype threat, and under-representation in AP courses are not. In the modified form of REM STEM offered here, oppositional culture and stereotype threat are conceived of as broader, contextual factors that operate on minority students at all points in their development. Indeed, these factors will be shown to flow from a broad historical cultural context shared by those minority groups which are typically underrepresented in STEM vocations. By placing K-12 experiences and K-12 outcomes within a broader, situated context, this modified version of REM STEM borrows from Perna's (2006) model of college choice.

Perna's (2006) model also recommended other factors relating to the K-12 experience which are not included in REM STEM. Perna suggested that "such institutional agents as teachers, counselors, and middle-class peers provide access to resources" (p. 118) which have the potential to impact K-12 outcomes. 
In addition, "institutional structures that limit the ability of working-class minority students to develop 'trusting' relationships with institutional agents" and that foster the "short-term duration of interactions" (p. 118) further restrict the ability of those students to develop college-bound aspirations and credentials. For this reason, teacher-to-student ratio is included as a variable within the school funding and resources construct. In addition, the economic and ethnic makeup of the school are included as important control variables, as is academic momentum-a construct described in some detail in the final methods section of this proposal. Finally, school size is notably absent from both the Museus (Museus et al., 2011) and Perna's models despite the relatively strong body of empirical evidence showing that smaller schools reliably serve under-served students more effectively both in terms of academic achievement and in terms of students' sense of engagement with the school community.

As shown in Figure 2, the literature review is organized around three primary factors, labeled F. Factor F3 is further broken into several components, labeled F3-1 through F3-5. The review begins with important contextual factors which help to frame the discussion of minority success in AP Calculus. First, Ogbu's $(1982,1992,2004)$ theory of social discontinuity is described and linked to oppositional culture theory, which are, in turn, based on Allport's concepts of the formation of prejudice and the phenomena of stereotype threat. Next, the impact of parental expectations and support is discussed and linked to Pierre Bourdieu's widely cited concepts of cultural capital and habitus. The identification 
of impactful school-level variables constitutes the primary focus of the review.

Finally, central questions around differential minority achievement in AP Calculus are revisited and clarified.

\section{Organization of Literature Review}

F3: The K-12 Experience

Funding and resources

Teacher qualifications

College prep participation

School size

School choice and

governance

Academic momentum

\section{Demographic Makeup}

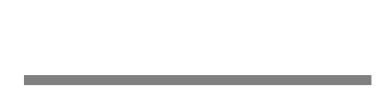

F1: Historical and Cultural Context Social Discontinuity Theory Oppositional Culture Strereotype Threat

\begin{tabular}{|c|}
\hline Participation and \\
achievement in AP \\
Calculus
\end{tabular}

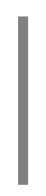

F2: Parental

Support and Expectations

Cultural and Social

Capital

Schematic representation of organization of literature review showing the three primary components of interest.

Figure 2. Organization of literature review.

\section{F1: Historical Social Contextual Factors}

Historical/social contextual factors are those factors that impact all members of all groups at all points in their development, although not in the same way. Applied to those minorities which are underrepresented in STEM 
disciplines, these are factors which serve to differentiate the experience of members of the minority groups from the experiences of the dominant, nonminority society. Of course, responses to these factors vary widely from individual to individual. Indeed, "group differences . . . are certainly less marked than they are generally supposed to be. Differences within groups are almost always greater than differences between groups" (Allport, 1954, p. 139). Nonetheless, certain generalities can be described. In the sections that follow, the seminal contributions of Gordon Allport, John Ogbu, and Pierre Bourdieu to our understanding of the minority experience are discussed. Within the model described earlier, it is supposed that the influences illuminated by these scholars pervade the $\mathrm{K}-12$ experience of minority students and therefore strongly impact K-12 outcomes, including the AP Calculus outcomes identified for investigation.

\section{The Formation of Prejudice and Out Groups}

The work of Allport (1954) on the fundamental nature of prejudice forms the backbone of our current understanding of the minority experience. Prejudice, Allport suggested, is simply the tendency to judge or categorize an event or person based on previous experience. Prejudice thought of in this is way is a natural, and even necessary, human activity. No one person can possibly encounter every instance of an event or take the time to carefully consider the characteristics of each encounter one by one. "We cannot let our ignorance detain us in our daily transactions" (p. 9). When this necessary human tendency is combined with a second, equally common tendency-the tendency to 
overgeneralize-conclusions about others become possible which are not necessarily supported by direct experience. A negative interaction with a single member of a specific ethnic group, for example, can quickly become generalized to apply to all members of that group. Ethnic prejudice emerges, and while ethnic prejudice could potentially be both positive and negative, Allport maintained that "ethnic prejudice is mostly negative" (p. 7). That is, where it is present, ethnicallybased prejudices most often ascribe negative traits to members of specific groups.

Human beings tend to cohere in homogeneous groups. Ethnic communities commonly form in cities and in rural areas alike. Certain geographic regions become known for the high concentrations of specific ethnic groups, leading to Pennsylvania Dutch, for example, or Minnesotan Scandinavians. There is nothing in this that is necessarily bad. This tendency is not based on an automatic hatred or distrust of other groups, but on "nothing more than convenience. There is no need to turn to out groups for companionship" (Allport, 1954, p. 17). Human beings have a natural affinity for the familiar. The familiar provides the "indispensable basis of our existence" (p. 29), and this fact, coupled with the natural tendency to overgeneralization and the capacity for hostility, leads to the formation of in groups and out groups built along lines of ethnicity.

Often, but not always, membership in ethnically-based in groups and out groups is identifiable through physical characteristics like skin color. Although there is no physiological basis for this, physical characteristics are assumed to 
equate to specific group characteristics, making membership within certain ethnic groups especially persistent and difficult to modify. Whether for good or for ill, an individual cannot easily leave behind their skin color or the shape of their nose or eyes. This fact forces members of ethnically-based out groups to adopt specific coping strategies in order to advance their own survival.

Allport (1954) identified a number of coping strategies which might be adopted by members of an out group depending upon the tendencies of the individual. An individual who is basically "extropunitive" might be expected to adopt attitudes of excessive suspicion, aggression and revolt, or "slyness" and "cunning." An individual who is basically "intropunitive" might be expected to deny membership in their own group (to "pass for White" for example), or to adopt modes of clowning, passivity, or self hate. Each of these strategies are adopted as a defense against negative prejudices levied against out groups on a continual basis, prejudices that the individual cannot escape due to the permanent nature of their own physical features.

In groups and out groups are sometimes not permanent. During the early 1900s, Turks were widely reviled in the United States even though most Americans had never met a Turk. At various points in history, Irish, Italian, Chinese, and Japanese Americans were considered to be inferior. Industry titan Henry Ford (1926) wrote openly in the mid-1920s of "first class men" who were almost always of Germanic or English heritage and who eschewed the traditions and tendencies of Eastern Europeans. Indeed, H. Ford employed a number of 
workers in his so-called "Sociological Department" whose job it was to train Eastern European employees into the modes of their "first class" Northern European coworkers (Meyer, 1981).

While some of these prejudices have faded over time, other ethnically and racially based prejudices continue to be powerful forces in American society. For example, economic and educational outcomes for Irish Americans are indistinguishable from those of German Americans. Indeed, both are grouped in most cases under a single ethnic category-White. The same cannot be said for Native Americans, Blacks, Hispanics, and Asian Americans. Although significant variation exists within each of these large and diverse groups, in general Native Americans, Blacks, and Hispanics typically academically under achieve relative to their White classmates while students who identify themselves as Asian typically over perform their White and Asian classmates. The differential experiences of these minority groups, especially immigrants, was a topic of investigation taken up by late anthropologist John Ogbu beginning in the 1970s.

\section{Voluntary Versus Involuntary Immigrants}

Ogbu $(1982,1992,2004)$ made a distinction between two types of immigrants-voluntary and involuntary-and postulated that the experiential differences between these two groups accounted for many of the differences in outcomes observed. Under this conception, minority groups who immigrated involuntarily or who became members of an ethnic out group by virtue of military conquest and occupation would be expected to have experiences that are 
different from those minorities who arrived in the United States by choice. Most Asian Americans immigrated to the United States in response to economic opportunities, most notably the discovery of gold in California in the mid-1800s. In a similar fashion, Irish citizens immigrated to the United States to escape economic privation in their own country and in hopes of securing economic security for their families.

The same cannot be said for Blacks, Hispanics, and Native Americans. Blacks arrived in large numbers on the North American continent through the mechanism of slavery, while Hispanics in California and Native Americans across the continent remained in place and become out groups as the result of military conquest. As a result, Ogbu (1982) argued, these groups experience formal schooling differently.

Specifically, Ogbu $(1982,1992)$ proposed the existence of three types of cultural discontinuities that children might encounter differentially as they enter and pass through the formal educational system in the United States: universal discontinuities, primary discontinuities, and secondary discontinuities. Universal discontinuities are those discontinuities which all children encounter as they pass from their home environments into formal school environments. Learning to raise your hand before speaking and learning to do mathematics which are abstracted from specific events fall into this category.

Primary discontinuities are discontinuities which result from "cultural developments before members of a given population came into contact with 
American or Western White middle-class culture" (Ogbu, 1982, p. 293). These sorts of discontinuities are often encountered by members of immigrant groups or by non-Western groups who are introduced to Western-style formal education systems. An emphasis on competition or punctuality fall into this category. In the case of immigrant populations, a mismatch between a student's native language and the language of the dominant culture constitutes one of the most salient primary discontinuities.

Secondary discontinues, in contrast, "develop after members of two populations have been in contact or after members of a given population have begun to participate in an institution" (Ogbu, 1982, p. 298). Secondary discontinuities, therefore, develop as a "response to a contact situation" (p. 298), in contrast to primary discontinues which exist as a priori conditions to the contact. Ogbu (1982) suggested that secondary discontinues are especially likely to develop under stratified social conditions such as exist under caste systems, colonial stratification, or slavery. In cases where subordinate group members are prohibited from participating in the dominant culture through various legal or societal mechanisms, secondary discontinues develop in order to maintain group identity, in order to maintain group security, in order to assist group members in obtaining the benefits of full societal membership, or in order to oppose the dominant society.

In general, then, primary and secondary discontinues are likely to be experienced by involuntary immigrants in a society, while voluntary immigrants 
are likely to experience only primary discontinuities. This is because voluntary immigrants are more likely to experience cultural differences as challenges to be overcome on their way to the full benefits of citizenship. In contrast, because involuntary immigrant groups are kept from the full benefits of citizenship through legal or societal mechanisms, secondary discontinues develop which are most often oppositional in nature. This construct sorts well with Allport's (1954) earlier conceptions of defense mechanisms adopted by out groups. Members of ethnic out groups who are primarily extropunitive might be expected to develop attitudes of "aggression and revolt" (Allport, 1954, p. 157), a phenomena later called "oppositional culture" by Fordham and Ogbu (1986).

\section{Oppositional Culture and the Burden of Acting White}

Oppositional culture theory was inaugurated in the mid-1980s by the work of anthropologists Ogbu and Fordham (Fordham \& Ogbu, 1986; Ogbu, 1982, 1992, 2004). Oppositional culture theory is most often applied to Blacks because members of this group arrived in North America 400 years ago as slaves. For most of their history, Blacks have encountered strong legal, societal, and religious restraints based on their ethnicity. In response Black culture has developed various religious beliefs, communication and language forms, and patterns of social relations which "constitute a more or less distinct lifestyle ... In general, Black culture is defined in opposition to White culture" (Ogbu, 1982, p. 299). One specific example of this is a form of "social inversion" in which 
mainstream words such as "bad" take on precisely the opposite meaning within Black culture (Ogbu, 1992).

Fordham and Ogbu (1986) formally applied Ogbu's ideas to the context of schooling. In it, these authors described the tendency for Black school childrenand especially students in high school-to ascribe academic success to White culture and to describe those Black students who achieved good grades or high test scores as "acting White." The associated affective dissonance and stress constituted, according to Fordham and Ogbu, "one major reason that Black students do poorly in school" (p. 177). "The perception of school as a subtractive process" write these authors "causes subordinate minorities to 'oppose' or 'resist' academic striving" (p. 183).

In their ethnographic analysis of 33 African American students attending an urban high school in Washington, DC, Ogbu and Fordham documented a number of behaviors which were identified by Black students as acting White, including spending time at the library, being on time, and getting good grades or trying to. Since acting White stood in opposition to being a part of Black culture, these activities were labelled as unacceptable for Black students, presumably producing reduced levels of academic performance for Black students. This extension of Ogbu's earlier description of cultural discontinues formed a powerful explanatory frame work for investigators over the next three decades. Indeed, "one would be challenged to find a set of ideas aimed at explaining racial/ethnic 
gaps in school performance which has sparked more interest and debate in the past 30 years" (Downey, 2008, p. 110).

Ogbu (2004) documented similar experiences for Black students in Oakland, San Francisco, and Shaker Heights, Pennsylvania. D. Ford, Grantham, and Whiting (2008) reported corroborating results in their examination of gifted Black students in 3 school districts. These students reported that acting White meant doing well in school, taking advanced courses, and being smart while "acting Black" meant academically underachieving. The majority of these students reported not trying as hard as they could in school and experiencing significant levels of peer pressure to "act Black". Ford's results are especially intriguing given that the students being investigated had, to some degree, defied the Black norms they reported in favor of academic achievement. Corroborating results have also been reported by other investigators including Farkas, Lleras, and Maczuga (2002) and Irving and Hudley (2008).

Other investigators, however, have raised serious objections to oppositional culture theory. For example, using the Maryland Adolescence Development in Context Study, A. Harris (2006), identified several specific incongruities between her results and Ogbu's $(1982,1992)$ theoretical approach. First, contrary to Ogbu's formulation, A. Harris (2006) found that Black students perceived greater potential gains to education and held higher aspirations than White students. Second, Black students reported a more positive affect and demonstrated less resistance toward school than White students, once again 
contradicting Ogbu. Additionally, Black students reported spending as much or nearly as much time on homework as do White students. Finally, Black students in A. Harris' (2006) sample reported no additional negative peer interactions around academic achievement than did White students once students' socioeconomic status was controlled for. In a later, related study, A. Harris (2007) reported that previous academic performance and not factors related to oppositional culture were most predictive of academic achievement for African American students. Similar results were reported by Ainsworth-Darnell and Downey (1998) in their analysis of National Educational Longitudinal Study. "African Americans are more pro-school and are more likely to esteem their highachieving peers than are Whites" (Ainsworth-Darnell \& Downey, 1998, p. 551), a result which contradicts one of Ogbu's central claims and which Downey (2008) underlined in his review of Ogbu's ideas.

Some investigators have gone further, suggesting that, in addition to lacking empirical support, oppositional culture theory fosters White supremacist attitudes, equates Black culture with a culture of poverty, and relieves the White, dominant society of the burden of reform by placing the onus of blame for the achievement gap within the Black community (Ainsworth-Darnell \& Downey, 1998; Downey, 2008; Lundy, 2003). In a paper published posthumously in 2004 , Ogbu responded to his critics, claiming that so-called oppositional culture theory was in fact an overly narrow reinterpretation of his much broader culturalecological theory. Acting White in school, he argued, was just one component of 
his model in which multiple factors interact to produce the modern Black experience. Ogbu (2004), referring to Fordham and Ogbu (1986), wrote that "critics construct and study a different problem than the one we laid out in the the joint article" (p. 2). Despite the controversy, however, investigators continue to advocate for oppositional culture theory as a valuable analytical lens, not least because it "highlights the importance of educators and school personnel in K-12" (Museus et al., 2011, p. 37) in addressing the minority gap in STEM outcomes.

Oppositional culture bears a close relationship to Allport's (1954) "ego defensive" mechanisms deployed by out-group members and, in fact, can be considered as another of those same mechanisms. These mechanisms are undertaken as a means to ameliorate the ill effects of negative prejudices and to create a livable environment for members of the out group. They arise because "one's reputation, whether true or false, cannot be hammered, hammered, hammered into one's head without doing something to one's character" (Allport, 1954, p. 142). Ego defensiveness in one form or another can therefore be expected to emerge where out groups exist, especially, as Ogbu postulated, when those out groups are formed from groups of involuntary immigrants.

The mechanisms described here are more or less conscious responses to prejudice. Those who deploy them often do so intentionally and knowingly. Individuals can (and do) sometimes switch these responses off as the situation requires. In the late 1990s investigators began to document a different sort of response to prejudice, one which seemed to operate continually and 
unintentionally and to operate on members of in groups and out groups alike. This phenomena, called stereotype threat, has been reliably shown to negatively impact performance of all sorts and is described in the next section.

\section{Stereotype Threat}

Being the target of a negative stereotype "represents a significant threat to self regard" (Stone, Lynch, Sjomelig, \& Darley, 1999, p. 1213). Ego defensive mechanisms arise in response to this threat because "no one can be indifferent to the abuse and expectations of others" (Allport, 1954, p. 139). On the contrary, when persistent inferiority images are internalized, members of the out group adapt. Stereotype threat constitutes one form of response to prejudice. However, unlike Allport and Ogbu's defensive mechanisms, stereotype threat is not activated as the result of the internalization of negative messages and images. Instead, it is a response to the "immediate situational threat that results from the broad dissemination of negative stereotypes about one's group" (Steele \& Aronson, 1995, p. 798). Performance is suppressed because participants fear being judged or fulfilling negative stereotypes through poor performance. Ego defensive mechanisms are manifest only by members of disparaged out groups or, in the case of oppositional culture, by involuntary immigrants. In contrast, stereotype threat "can befall anyone with a group identity about which a negative stereotype exists...he need not even believe the stereotype" (Steele \& Aronson, 1995, p. 798). 
For example, Black athletes are generally perceived to be better natural athletes compared to their White team mates, while White athletes are generally perceived to be smarter or have a higher "sports IQ" (Stone et al., 1999). Stone et al. (1999) demonstrated the effect of these stereotypes on the athletic performance of 82 undergraduate students enrolled at Princeton University. One sub group of this experimental pool was told that the task to be performed (putting a golf ball) was a measure of native athletic ability while a second sub group was told that the task was a measure of one's ability to think strategically about the task. Black students significantly outperformed the White students when they believed the task was a measure of athletic ability but underperformed the White students when they believed the task was a cognitive measure. The reverse was true for White students. It is also interesting to note that all of these students had been matriculated to Princeton University and so were necessarily academically successful students in the first place. The Black students who participated in the experiment apparently believed themselves to be at an intellectual disadvantage despite having been admitted to an Ivy League school.

This effect is also notable in tests targeted specifically to intellectual ability. Steele and Aronson (1995) demonstrated the effects of stereotype threat on the performance of Black and White students on several measures of intellectual ability and test anxiety. When students were told that the test they were about to take was not meant to measure intellectual ability White students outperformed Black students, but only slightly. In contrast, when students were told that they 
were about to take a diagnostic test, White students significantly outperformed Black students. This effect became more pronounced when the level of difficulty and frustration experienced by the students while taking the test became greater. Black students also produced a larger number of self-handicapping responses to survey items (i.e., the test is unfair or I didn't get much sleep) than did White students on tasks which were introduced as diagnostic tasks. These investigators concluded that "making Black participants vulnerable to judgement by negative stereotype about their group's intellectual ability depressed their standardized test performance" (p. 808).

Aronson, Lustino, Good, and Keough (1999) showed that this effect can be activated even when the participants are not members of a stigmatized group. In their investigation they found that White males who were identified as being moderately or highly math capable performed less well on a test of mathematical ability when a racial stereotype was activated. Specifically, when the test was prefaced with "A good deal of research indicates that Asians consistently score higher than Whites on standardized tests of math" White students' performance was depressed. This effect was particularly pronounced for those students rated as highly math capable and who reported a great deal of concern over their performance, suggesting that students who are more invested in their performance are more strongly affected by stereotype threat.

Spencer, Steele, and Quinn (1998) demonstrated this effect for female undergraduate students enrolled at the University of Michigan. All participants for 
this study were highly successful math students and completed test items which were taken directly from mathematics section of the Graduate Record Exam. When participants were told that the math test they were about to take typically generated gender-based differences in scores, female students significantly underscored male students. However, when students were not told that the test typically generated gender-based differences, the score differential disappeared. In a separate investigation, the test items were somewhat easier and the participants were explicitly told that the test did not generate gender-based differences in scores. In this case, gender-based differences disappeared, suggesting that removing stereotype threat improves test performance. Similar results were reported by Wei (2012) in his large scale analysis of National Assessment of Educational Progress (NAEP) scores. Where mathematics test items were proceeded by statements that activated negative, gender-based stereotypes, female students performed more poorly than male students. Interestingly, when items were primed with statements that attempted to activate positive stereotypes about girls in mathematics, no effect on scores was observed.

Croizet and Claire (1998) extended this concept to membership in a specific socioeconomic class. In this investigation, less affluent French students underperformed more affluent students on a written test of verbal ability when told that the test diagnosed intellectual capability and when told that relative poverty has a negative impact on test performance. Those differences 
disappeared when the test was introduced as non-diagnostic and when negative stereotypes about SES were not activated prior to the test. The less affluent students also produced more self-handicapping responses on survey items designed to measure test anxiety.

The results cited above indicate that stereotype threat strongly impacts performance on assessments of verbal ability, mathematical ability, and even athletic ability. The power of stereotypes appears to operate across lines of ethnicity, gender, and socioeconomic class and can be activated even when the test subjects are not members of a disadvantaged class. Additionally, investigators have demonstrated that stereotype threat reduces working memory (Schmader \& Johns, 2003) and increases blood pressure in testing situations (Blascovich, Spencer, Quinn, \& Steele, 2001). The impact of societal stereotypes on the performance of minority students in STEM disciplines can therefore be expected to be pervasive and pernicious.

\section{Summary}

Allport (1954) provided the seminal work describing the development and results of prejudice. Prejudice, or pre judgment, is the result of the natural human tendency to evaluate and categorize based on previous experience. Prejudice becomes negative when pre judgment is combined with a second human tendency-the tendency to overgeneralize-and with the human capacity for hostility toward others. In groups and out groups form because humans tend to cohere in homogeneous groups out of convenience and because the familiar 
forms the psychological basis for existence. When hostile overgeneralization is directed toward an out group, prejudice in its fuller sense emerges.

In response to negative prejudice, out groups develop one or several ego defense mechanisms. In some cases, when an out group is formed as the result of involuntary immigration, a specific sort of ego defense mechanism emerges in which the out group defines its culture in opposition to the dominant group. Defense mechanisms of this sort can sometimes serve to interfere with the participation of the out group in the dominant culture, and sometimes, as in the case of oppositional culture, this is intentional on the part of the out group. In contrast, stereotype threat is a response to prejudice that is not intentional and which serves only to reduce performance. It can be activated whenever an individual is a member of stereotyped group and therefore applies to members of in groups and out groups.

These underlying mechanisms and responses to prejudice are taken to be foundational in the model presented earlier. Scarcely can a member of certain minority groups enter a "store, restaurant, movie, hotel, amusement park, school, train, boat, or plane ... without wondering uneasily if he will suffer insult and humiliation" (Allport, 1954, p. 140). Race consciousness can emerge at a young age. Referring to the work of sociologist Mary Goodman, Allport (1954) reported that Black nursery-aged children were "troubled by their first awareness of handicap" and that "some of them were already in various ways defensive, over 
reactive, and tense as a consequence of their vague feeling of disadvantage" (p. 111).

A wide range of response mechanisms have been documented. Rap music, for example, has "revolved around the concept of battling" (Au, 2005, p. 210) the paradigms and assumptions of the dominant culture. "The Discourse of rap music is in the midst of a battle with that of education where rap music resists and critiques education as a dominant and domineering Discourse" (Au, 2005, p. 210). While some ascribe the persistent underachievement of minority students, and especially Black students, to direct and intentional opposition, others attribute the achievement gap in part to students' concrete beliefs about the value of education (Herman, 2009), the internalization of teachers' views of minority students (Fisher, 2007), or the result of schools structured to perpetuate patterns of discrimination (Harry \& Anderson, 1996; Kenyatta, 2012). The force which drives all of these responses is prejudice which is seen to be pervasive and persistent in human societies.

Having laid this foundation, we turn now to the role of parental support and expectations which Museus et al. (2011) conceived of as impacting the experience of minority students throughout their STEM careers. The following sections show that families are the primary locus for the promotion and propagation of social, cultural capital, and economic capital and, as such, are pivotal in the development of student aspirations and interests. Furthermore, parental expectations define students' sense of the importance of formal 
education and therefore strongly impact important educational outcomes like early school leaving and course taking.

\section{F2: Parental Expectations and the Cultural Capital Explanation}

Allport (1954) supposed that individuals exist within a set of concentric group identities. An individual might be a part of a nation, a city, a neighborhood, and a family simultaneously, but will not identify with all of those groups with the same level of commitment. Only a few are likely to be self-schematic for the individual, and some will be viewed as casual associations easily discarded. In Allport's typology, the family forms the inner most circle. Allport wrote, "psychologically, the familiar provides the indispensable basis of our existence" (p. 28). Casual consideration of the matter immediately suggests that family influences will have strong impacts on a student's educational outcomes and choices, a finding supported by empirical evidence. The following sections present evidence supporting this assertion and describe one possible theoretical explanation, Pierre Bourdieu's theory of cultural capital.

\section{The Effect of Parental Expectations on Achievement}

The importance of parental expectations and involvement to student achievement has been broadly recognized in the literature, and in increasing measure over the last two decades (Jeynes, 2005). Hara and Burke (1998) went further, suggesting that "few other ideas or resources would likely impact the learning environment as much as having parents become, in effect, extensions of the teachers and their classrooms" (p. 9). In his meta-analysis of 21 studies on 
the effects of parental involvement on the achievement of minority students, Jeynes (2005) identified a number of common results. First, parental involvement positively and significantly affected student achievement for all the ethnic groups under consideration. Second, effect sizes from 0.01 to 0.74 but were in general greater than 0.2 standard deviations. Where academic achievement was measured in terms of GPA, effect sizes were somewhat smaller, but still significant. Third, Black and Latino students appeared to benefit more strongly from parental involvement than did Asian students, a finding which Jeynes ascribed to the greater likelihood that students from the first two groups come from single-parent homes.

Corroborating evidence regarding the important of parental influences has also been provided by a number of other investigators. For instance, Russell and Atwater (2004) interviewed 11 Black Biology majors during their senior year of college regarding their experiences in high school science. All 11 participants "expressed that it was either 'understood' or an expectation within the household that they would attend college" (p. 699). Some participants indicated that their parents' occupations and their parents' influences on their student habits were critical to their persistence in science. In a similar study, Moore (2006) examined responses to biographical questionnaires gathered from 42 Black university students majoring in engineering. Like the students in Russell and Atwater's pool, the participants in Moore's study uniformly reported on the importance of parents' occupations, influences with regards to early exposure to science and 
engineering, and direct support with regards to the college process. One participant also described the influence of cousins who had themselves graduated from Harvard.

In a large, 5-year study of Black and Hispanic undergraduate students who were majoring in Physics, Fries-Britt, Younger, and Hall (2010) drew similar conclusions with regards to parental involvement and influence in the lives of science-successful students. Many of the 110 participants reported that parents played a critical role in introducing science activities into the home. In addition, these researchers found that often the influence and encouragement of $\mathrm{K}-12$ teachers were pivotal. Hrabowski (2003) interviewed more than 100 families affiliated with the Meyerhoff Scholars Program at the University of Maryland in order to determine "what actually works in bringing up academically successful minority students" (p. 45) who were interested in the sciences. Like other investigators, Hrabowski found that parents who held high expectations for their children, emphasized the importance of reading, and interacted regularly with teachers most successfully contributed to the academic success of their children. Hrabowski also reported that the modes of discussion around race, prejudice, and racial identify that occurred in the home were important.

In a much larger, quantitative study which sampled 1,373 immigrant students and 16,539 native students (students born in the United States) from 4 ethnic groups, Hao and Bonstean-Bruns (1998) found that parental involvement in school learning at home had a strong, significant, and positive impact on 
students' expectations for themselves and that parental involvement in other types of learning activities had a strong, significant, positive effect on parents' expectations for themselves. Parents of immigrant students had higher expectations for their children than native, White students, with parents of immigrant Chinese and Korean students having the highest expectations. The shared effect of family expectations on average academic achievement was "positive in sign, large in magnitude, and high in significance" (p. 189).

The effect of variables relating to the home environment on math achievement specifically has also been documented. Crane (1996) showed that home environment variables had a stronger impact on students' math scores than did the family's SES, results in general corroborated by Muller (1998). Impacts of parental involvement have similarly been demonstrated on reading achievement and have been shown to hold regardless of the parents' educational or economic background (Jeynes, 2005). These investigations, taken together, point to a broad general consensus: parental and family expectations play a strong role in the academic achievement of minority students.

\section{The Cultural Capital Explanation}

French sociologist Pierre Bourdieu offered one of the most often cited-and most often refuted-theoretical explanations for the importance of parental activities to student achievement known as the theory of Cultural Capital and its transmission through families. Capital in its general sense is accumulated labor and "the principle underlying the imminent regularities of the social world. It is 
what makes the games of society ... something other than simple games of chance" (Bourdieu, 2002, p. 46). Since native human abilities are presumably normally distributed, whenever the possessions and qualities of life are not normally distributed we see the effects of accumulated capital at work.

In his widely cited Forms of Capital, Bourdieu (2002) postulated three forms of capital: economic capital, cultural capital, and social capital. The first of these is the simplest. Economic capital is that form of capital that is "immediately and directly convertible into money" (p. 47). In the context of schools, disparities in school funding and the differences in physical facilities available amount to differences in economic capital.

The second of these-cultural capital-is the accumulated "dispositions of mind and body," the accumulated store of "cultural goods," and the accumulated store of credentials and qualifications obtained by and individual or a community. For Bourdieu (2002), it is the "domestic transmission of cultural capital" that represents the "most determinant educational investment" or, in other terms, that "ability or talent is itself the product... of cultural capital" (p. 48). Within this framework, a high school diploma simply represents the institutionalization of cultural capital and a partial reconversion of cultural capital into economic capital. Bourdieu's third form of capital is social capital and represents the total accumulated volume and depth of a person's social network. A person with a great deal of social capital is well connected with those in society with power. Just as cultural capital can be institutionalized "in the form of educational 
qualifications" (p. 47), cultural capital can be institutionalized "in the form of a title of nobility" (p. 47).

For Bourdieu, the family is the central mechanism for the transmission of cultural capital, a notion that has been "well documented" within the literature (De Graaf, De Graff, \& Kraaykamp, 2000). The possession of cultural capital is, in turn, what is rewarded within formalized educational systems. Certain traits such patterns of language or dress are forms of cultural capital. Schools require that students possess these forms, but, in general, do not provide them. Instead, access to "academic rewards depends on the cultural capital passed down by family, which, in turn, is largely dependent on social class" (Dumais, 2002, p. 44). Among those workers in the upper and middle class, teachers in particular possess and value cultural capital (DiMaggio \& Useem, 1998; Dumais, 2002). Students who possess the appropriate cultural capital are more likely to relate easily to their teachers and be positively sanctioned in school (De Graaf et al., 2000; Dumais, 2002).

However, the possession of cultural capital is, of itself, not sufficient. One's disposition or sense of where one fits within society is also important, a concept called "habitus" (Dumais, 2002). Habitus is "internalizing the social structure and one's place in it" (Dumais, 2002, p. 46) and bears some relationship to Allport's (1954) ego defensive mechanisms. In both cases, the individual develops a sense of what is possible and effective within the existing social structure and adapts behaviors and actions accordingly. In both cases, the pervasive nature of 
prejudice in society forms the field within which actions are taken. If the field is taken to be the school then actions within that field like trying to get good grades and turning in homework are strongly influenced by a combination of cultural capital and habitus (Dumais, 2002).

The intergenerational transmission of cultural capital is hypothesized by some to account for much of the persistence of the so-called achievement gap. In their analysis of school aged children within the Dutch public education, De Graaf et al. (2000) found that certain elements of parents' cultural capital-reading behaviors and linguistic patterns-were more important than those pertaining to the participation in "high brow" culture in providing children with educational advantages. Dumais (2002) also found that elements of cultural capital did have an effect on educational outcomes, but other factors like inherent ability and the family's SES were more important factors in their analysis. In a similar way, Sullivan (2001) found that elements of cultural capital relating to a student's intellectual skills like reading had a significant impact on academic achievement on standardized test scores. Somewhat in conflict with other results, Sullivan found "no support for the view that teachers are prejudiced against working-class pupils because of their lack of cultural capital" (p. 906).

\section{Summary}

Broad census exists around the notion that a student's family context strongly impacts academic achievement. Differences in the family's social and economic status and language proficiency in English affect direct measures of 
academic achievement, attitudes and optimism toward school work, and persistence in school (Caldas \& Bankston, 1997; Mickelson, 1990; Rumberger \& Larson, 1998). Minority students who are successful in science routinely report that strong family expectations around grades and college attendance were important in students' choice of STEM major in college and persistence in the major (Moore, 2006; Russell \& Atwater, 2004). Similar effects have also been demonstrated in large-scale statistical analyses (Hao \& Bonstean-Bruns, 1998) and have been noted to be important regardless of the parents' educational or economic background (Jeynes, 2005).

Bourdieu's theories of social capital and its inter-generational transmission through the family provide one explanation for how and why family context might so strongly impact school success. Within this framework, specific dispositions of mind and body plus certain goods and credentials are transmitted through familial interactions and rewarded within the school system. Students who possess appropriate cultural capital are positively sanctioned within the school system while students who do not possess the appropriate cultural capital find the school environment to be foreign and confusing (De Graaf et al., 2000; Dumais, 2002). While a number of investigators have found empirical evidence that at least some components of parents' cultural capital impact student achievement (Archer et al., 2012; DeGraaf et al., 2000; Dumais, 2002), others have suggested that Bourdieu's theory of educational reproduction is over simplified, suffers from lack of specific operationalization of important terms, and 
fails under the test of empirical evidence (Goldthorpe, 2007; Sullivan, 2001).

Whatever the underlying causal mechanisms, however, the evidence cited above suggests that what families and parents do matters. In the Museus et al. (2011) REM STEM model, parental expectations and involvement impact students at every stage of the STEM educational experience.

\section{F3: The K-12 Experience}

A student's eventual success in STEM educational and vocational experiences depends to a large extent on the strength of that student's K-12 academic preparation (Adelman, 1999, 2006; Bonous-Hammarth, 2000; Museus et al., 2011; Oakes, 1990). However, Black and Hispanic students are consistently under enrolled in honors and college-preparatory classes, consistently underperform on standardized measures of academic achievement during their K-12 years, and leave school early at higher rates as compared to their White classmates (Callahan, 2005; Chapman et al., 2010; Coley, 1999; Flores, 2007; Gross, 1993; Hallinan, 1994; S. Lewis et al., 2010, 2011; Oakes, 1985; Solorzano \& Ornelas, 2002; Zuniga, Olson, \& Winter, 2005). These results constitute important economic and ethical challenges to America's K-12 STEM educational system. Beginning with a more complete description of school outcomes for Black and Hispanic students, the following section describes important school-level factors impacting the academic experience of minority students. Specifically, the following school-level factors are discussed: F3-1) Funding and resource disparities; F3-2) Disparities in teacher qualifications and 
expectations; F3-3) Tracking and differential enrollment in college-preparatory courses; F3-4) School size; and F3-5) School governance.

\section{Educational and Economic Indicators}

Black and Hispanic students are disproportionately exposed to poverty, economic instability, and access to health care as they progress through childhood. Approximately $35 \%$ of Black students and $27 \%$ of Hispanic students enrolled in Council of Great City Schools live below the poverty line (S. Lewis et al., 2010) as compared to $10 \%$ for White students. Poverty, in turn, creates other realities for some minority students. About $12 \%$ of Black students and $17 \%$ of Hispanic students enrolled in Council of Great City Schools were not covered by private or government-sponsored health insurance and approximately $40 \%$ of these children lived in households where no parent has permanent, full-time year-round employment (S. Lewis et al., 2011). A disproportionate fraction of minority students also live with parents who are relatively less well educated. About $70 \%$ of all Hispanic students and $45 \%$ of all Black students enrolled in Great City Schools live in households in which the highest level of educational attainment by either parent is a high school diploma or less (S. Lewis et al., 2010, 2011).

Minority students also consistently under achieve compared with their White age mates on standardized measures of academic achievement. Blacks and Hispanics score approximately $10 \%$ lower on the NAEP reading assessment in the fourth and eighth grades and nearly $20 \%$ lower on the NAEP assessment 
of mathematics in those grades. Furthermore, White students living in poverty outscore both Black and Hispanic students who are not living in poverty, suggesting that SES alone is not sufficient to explain the persistent achievement gap. Similar trends are also observed on SAT scores. In 2009, Black males scored 120 points lower and 104 points lower on the SAT mathematics and critical reading sections respectively (S. Lewis et al., 2010). Similarly, Hispanic students scored 60 to 80 points lower than their White classmates on the 2010 SAT assessments of mathematics and critical reading (S. Lewis et al., 2011).

A host of additional metrics further demonstrate the disadvantaged position that many minority students are in as they approach their adult lives. Hispanic and Black students are much less likely to finish high school and much less likely to attend two and four year colleges. Compared to their White classmates, they are less likely to participate in extra-curricular activities, more likely to be suspended from school, and more likely to be retained or "held back" a grade level during their K-8 school years. Minority students are much less likely to take AP classes and much more likely to attend schools which experience a higher rate of violent incidents between students (S. Lewis et al., 2010, 2011).

Hall (2013) noted a large number disparities in opportunities in outcomes between White students and underserved minority students. As noted earlier, Black and Hispanic students dramatically underperform on measures of math and reading proficiency with respect to White students. Hall also noted, however, the students from these groups face a large number of disparities, including a 
teacher quality and training gap, a gap with respect to high level curriculum, an employment opportunity gap, and a school funding gap. Indeed, Hall suggested that the achievement gap is only the symptom and net effect of a multitude of other gaps faced by underserved minority students.

These indicators, taken together, paint a convincing picture. At first blush, it seems remarkable that minority students achieve to the degree that they do given the wide range of barriers they sometimes face. In fact, disadvantaged minority youth often remain more optimistic about their future and retain higher levels of educational aspirations compared to their disadvantaged White peers (Kao \& Tienda, 1998), a testament to the resilience of minority students and the communities they grow up in. A host of school-level factors give rise to the indicators cited above and ultimately create the under-representation of minorities in the college-enrolled population in general, but especially in STEM fields. In the following sections these school-level factors are identified and described.

\section{F3-1: Funding Disparities}

Historically public schools in the United States have been funded through property taxes. Because families tend to sort themselves into homogenous groups of like median incomes, properties of lower value were owned and occupied by families of lower income (Card \& Payne, 2002). These tendencies, working in tandem, created wide disparities in per-student funding between districts, the wealthiest districts spending nearly three times as much per pupil as 
the most economically disadvantaged districts (Condron \& Roscigno, 2003). A survey of per-pupil funding by geographic area and state released by the United States Department of Commerce (2012) further bears this trend out.

In 1992 , total expenditures averaged $\$ 5,001$ per pupil for public $\mathrm{K}-12$ education in the United States. In 2010, that expenditure reached $\$ 10,615$ per student, reflecting an annual growth in funding of just under $4 \%$. This growth rate has been relatively constant, rising slightly at the close of the millennia and slowing slightly in 2009. In contrast, the geographic distribution of per-pupil funding varied widely in 2009 both on an inter-state and an intra-state basis. During the 2009-2010 school year, Oklahoma, Arizona, Idaho, and Utah spent less than $\$ 8000$ per pupil on average. During that same year, Alaska, Wyoming, New Jersey, and New York spent more than $\$ 16,000$ per pupil, or more than twice as much per pupil. However, state-by-state averages do not tell the whole story. Intra-state variations were even larger during that same school year. In Illinois, Arlington Heights township spent $\$ 18,060$ per pupil while Oswego spent $\$ 8,090$ per pupil. Albemarie County in Virginia spent $\$ 17,574$ per pupil while Bedford County spent $\$ 8,514$ per pupil (United States Department of Commerce, 2012).

In their analysis of public school funding systems and the reforms begun, in many cases, by court order, Card and Payne (2002) demonstrated that funding disparities have not been appreciably reversed. This result was not because the court actions of the 1980s had no effect on funding equity on the state level, but 
because local funding options more than made up the difference. Between 1972 and 1999 , funding per student across all states increased by an average of $46 \%$, with increases in states in which the funding formulas were either upheld or reformed by court order outstripping increases in states in which no court action was taken. During this same period, in states in which the school funding formula was found to be unconstitutional, state aid was redistributed to poorer districts, resulting in changing in spending equity that were "both statistically and economically significant" (Card \& Payne, 2002, p. 67). However, in many states these moves toward funding equity were "offset by widening inequity in local revenues between richer and poorer districts" (p. 67).

Card and Payne (2002) also showed that where funding gradients were larger between districts, the differences in both SAT participation and SAT scores were larger. According to these investigators, spending equalization had a "modest effect ... on the relative performance of students from more disadvantaged family backgrounds" (p. 79). In a similar vein, Payne and Biddle (1999) demonstrated that levels of school funding have "substantial and statistically significant" (p. 11) negative impacts on average student achievement and that these impacts operate independently from other variables like family SES, ethnicity, and curricular instruction. To demonstrate the magnitude of this effect, Payne and Biddle showed that students who attended poorly-funded school districts in the United States answered, on average, 35 questions correctly on the SIMS international math test while students from affluent districts 
answered, on average, 57 questions correctly, leading these researchers to conclude that the difference in academic achievement between well-funded and poorly funded schools is "huge" in terms of student achievement.

In their analysis of spending within a large, urban school district, Condron and Roscigno (2003) also demonstrated a significant link between school-level per pupil spending and achievement. In the 83 elementary schools these researchers analyzed, for every $\$ 1,000$ per pupil expended, scores on standardized measures of achievement increased between $6 \%$ and $10 \%$. This result, while somewhat weaker, also held when racial and class composition factors were included. These investigators also found that differences in conditions of the facilities and orderliness of the learning environment were the primary mediating variables in this effect.

These differences in funding also sometimes translate into lower salaries for teachers. In an analysis of four urban school districts, Roza, Hill, Selafina, and Speakman (2004) found that teachers in high-poverty schools were paid between $5 \%$ and $10 \%$ less on average than their colleagues in more affluent schools which served a lower proportion of minority students. These salary disparities were not created by an institutional intention to underpay teachers in those schools. Rather, labor contracts typically provide for added within-district mobility as a teacher gains seniority. Because, as Roza et al. noted, working conditions are often more challenging in low SES and high minority schools, teachers disproportionately transfer away when the opportunity presents itself. Condron 
and Roscigno (2003) reached similar conclusions, noting that teachers with higher levels of education were disproportionately assigned to schools with higher per-pupil funding and that this result was primarily mediated through school-level spending on facility maintenance and instructional resources. The net effect is a disproportionate representation of more junior or, in some cases, less qualified teachers in the very schools where the best educators are needed.

\section{F3-2: Disparities in Teacher Qualifications and Credentials}

Various investigators have also noted a difference in the level of teacher qualifications in high-minority versus low-minority and high-poverty versus lowpoverty schools. Relatively affluent, White students are more often taught by teachers with a higher level of demonstrated subject knowledge, more experience, and with appropriate licensing credentials. For example, in his analysis of the Schools and Staffing Survey released by National Center for Education Statistics in the early 1990s, Ingersoll (2002) found that $96 \%$ of those teachers who served in a relatively affluent, suburban White schools had regular teaching certificate while only $85 \%$ of those teachers in urban, less affluent, minority schools were certified to the same level. Similarly, Ingersoll documented differences in educational level and years of teaching experience between these two types of schools. In the affluent, suburban, White schools $58 \%$ of all teachers held master's degrees and $28 \%$ had more than 20 years of experience, while in urban, poor, high-minority schools $47 \%$ and $27 \%$ held master's degrees and had more than 20 years of experience respectively. Similar patterns emerged 
between high and low poverty schools and high and low minority schools in terms of teachers who have (or do not have) a major in the field in which they were teaching. For example, approximately $38 \%$ of those teaching math in lowpoverty schools majored in math compared to $28 \%$ in high poverty schools.

Bruno (2002) also noted a surprising association between a school's geographic and economic setting and its reliance on substitute teachers to deliver instruction. In his examination of schools in Los Angeles, Bruno demonstrated statistically significant positive correlation $(p<0.01)$ between teacher absenteeism and an area's rate of crimes against property, crimes against people, number of regular teachers without a teaching credential, dropout rate, and number of substitute requests that go unfilled. In contrast, academic performance was negatively correlated with teacher absenteeism. Bruno wrote, "the principal finding of this study is that teacher absenteeism is felt most unfavorably in urban schools, or in schools located in poor, low median family income geographic space" (p. 16). Bruno's study also has implications with regards to disparities in school funding, since high rates of teacher absenteeism translate into significant diversion of resources away from the classroom.

The National Science Board (2012) reached similar conclusions with respect to STEM teachers specifically. These investigators found that in-field, more experienced STEM teachers were more likely to be found in low-minority, high-income schools than in high-minority, low-income schools. For example, $95 \%$ of all high school math teachers in low-minority, high-income schools were 
teaching in field as compared to $83 \%$ in high-minority, low-income schools. STEM teachers in high-poverty schools had lower salaries and reported significantly higher rates of tardiness and class cutting and depressed sense of job security due to lower test scores in their schools. Additionally, pre-service student teachers in STEM disciplines were more likely to be working in lowminority schools than in high-minority schools.

These numerical indicators sort well with anecdotal evidence reported by a number of observers. Haycock (2002) wrote that "we sometimes see wonderful teaching, in all types of schools . . . but we often see dreadful teachingespecially in the highest-poverty schools" (p. 11). The famed school reformer and writer Kozol (2005) reported that in one urban school he visited "thirteen of the 15 teachers were 'provisionals"' (p. 143), that in another urban school district the only requirement to obtain a teaching position (the following day) was to "bring in your college transcript" (p. 145), and that at one high-poverty, high-minority school in Monterey, California "half of the teachers working at the school two years before had either left or been removed" (p. 170). One Boston fourth grader reported that "I see new teachers olmots [almost] every day" (p. 163), a descriptor that Kozol reported could have been applied to "any one of countless inner-city schools in the United States today" (p. 163).

It is clear, then, that disparities in teacher credentials, experience, and education are prevalent, consistent, and wide spread. Minority and less-affluent students suffer from inequitable distribution of teacher resources, a fact which 
investigators have shown to have significant, detrimental impact on achievement. In their analysis of school districts in North Carolina, Clotfelter, Ladd, and Vigdor (2007) found that teacher experience and teacher test scores had the most significant positive impacts on student achievement, though teacher credentialing also had a significant and positive effect. Of those teacher characteristics included, only the "quality" of ranking of the teacher's undergraduate institution had little to no impact on student achievement.

Croninger, Rice, Rathbum, and Nishio (2007) reached similar conclusions, reporting positive impacts of teacher's degree type and years of experience on first grade reading achievement. In contrast, however, these investigators were unable to demonstrate an effect of teacher credentialing. When teacher credentialing and experience were aggregated at the school level, no impacts on student achievement were evident, though other school-level contextual variables became important. These researchers also found that students' socioeconomic and ethnic features were important school-level factors. Students at more affluent, less diverse schools achieved at higher levels regardless of teacher qualifications.

In their meta-analysis of teacher qualification research published prior to 2003, Wayne and Youngs (2003), concluded that teachers' undergraduate coursework, teachers' previous tests scores, and the type of credentials teachers held all had positive impacts on student achievement in math. The relationship between student achievement and the ranking of teachers' undergraduate 
institution, where present, was positive. In general, these authors produced results which broadly agreed with the researchers previously cited. In short, teacher qualifications and credentials impact student achievement.

\section{F3-3: Tracking and Differential Enrollment in College-Preparatory Courses}

Another important factor limiting the success of minority students in the STEM pipeline is discriminatory practices with respect to tracking and placement into college-preparatory courses. The practice of tracking emerged in the early part of the $20^{\text {th }}$ century in response to a radically enlarged and diversified high school student body (Reese, 2005). It was hoped that by increasing the homogeneity of academic groups or tracks, educators and school planners would be able to more efficiently allocate resources. Although tracking was viewed as a mechanism through which all students could be more effectively taught material at the appropriate level, the arrangement was constructed from an elitist view of the purpose of education. In 1919, Cubberley wrote that students could roughly be divided into "slow," "average," and "gifted" children and that the primary aim of educational reform "has been that of providing better advantages for our gifted children ... The future welfare of this Nation depends in no small degree, upon the right education of our gifted children" (p. 526).

Schools and districts vary widely with regards to procedures and criteria used to place students into tracks as well as the number and identity of the tracks used. The number of tracks tends to vary with school size: larger schools tend to offer more tracks, smaller schools tend to offer few tracks (Hallinan, 1994). 
Hallinan (1994) also showed that while track placement depended strongly on standardized test scores and pervious academic performance, additional background factors such as student SES impacted track placement. These results were partly corroborated by Archibald, Glutting, and Qian (2009), who found that track placement depended on standardized test scores and eighthgrade grades. Archibald et al. and Hallinan disaggregated data by ethnicity into two categories-Black students and White students-and neglected other minorities or variations within those broad categories. Neither of these researchers found that ethnicity (Black or White) had a consistent, statistically significant impact on track placement. Hallinan also showed that these track placements, once made, were relatively durable through a student's academic career with fewer than $20 \%$ of math students and $30 \%$ of English students changing tracks during their high school careers. Once again Hallinan reported that ethnicity was not a significant predictor.

A number of researchers, however, have noted a wide disparity between the number and richness of course offerings available to minority and those available to non-minority students (e.g., Coley, 1999; Oakes, 1985; Sanders \& Holt, 1997; Solorzano \& Ornelas, 2002; Zuniga et al., 2005), differences are often rooted in tracking decisions. Burris and Welner (2005) noted that minority students are over-represented in lower tracks, even when SES is controlled for within the analysis, therefore strongly suggesting that ethnicity, and not simple academic merit or SES, has at least some effect. In her seminal study of tracking 
practices, Oakes (1985) noted a clearly disproportionate representation of minority and low-income students in lower, less academic tracks.

In an examination of a rural mid-western high school, Zuniga et al. (2005) demonstrated that $80 \%$ of those Hispanic students who scored above the school's mean on a test of academic aptitude were placed in the lowest, noncollege preparatory track while none of the White students in the category were placed into that track. Conversely, while $86 \%$ of those White students who placed below the school's mean were placed into the highest track, just $21 \%$ of Hispanic students in that category were placed into that same track.

A similar effect was noted by Solorzano and Ornelas (2002) and Campbell (2007) with respect to minority enrollment in AP courses in California high schools. These investigators noted first that AP courses were not uniformly distributed throughout the state of California and that relatively more affluent schools offered more AP courses than less affluent, more urban schools. This places minority students at an immediate disadvantage because those students tend to enroll in the latter category of school. These researchers also discovered that, where AP classes were available, Hispanic and Black students were underenrolled. In one school district in California during the 1995-1996 school year, Hispanic students represented $68 \%$ of the total enrollment but only $45 \%$ of the enrollment in AP classes. The same trend was observed for Black students who represented $13 \%$ of the total enrollment but only $4 \%$ of the total AP enrollment (Solorzano \& Ornelas, 2002). 
Darity, Castellino,Tyson, Cobb, and McMillen (2001) reported similar results in their analysis of high schools in North Carolina. These investigators developed an "equity index" for purposes of comparison in which the percentage of minority students enrolled in a particular course was divided by the percentage of minority students enrolled in the particular high school. Computed in this way, an equity index of 1.0 represents an absence of an achievement gap in a particular course. All of the courses in most of the schools analyzed showed dramatic rates of underrepresentation. Over $40 \%$ of the schools analyzed reported equity index values below 0.2 in AP English and AP Biology. Half of the schools in which AP Calculus was offered reported equity index values of less than 0.2 and, in some high schools, there was no minority enrollment in this course. Nor is this surprising result limited to North Carolina. In 2011 in Oregon, only 24 Black students and 87 Hispanic students took the AP Calculus exam, together representing just over $5 \%$ of the total exams for that discipline administered in Oregon that year. Similar trends also emerge on a national level and are detailed in the final section of this literature review.

In terms of entry into the college-bound pipeline, these practices are particularly detrimental to minority students. Solorzano and Ornelas (2002) noted that the average freshman at the University of California Los Angeles in 1997 had a GPA of 4.15 and had taken 15.9 AP or honors courses. Students who attended schools in which AP or honors classes were not offered or who had been tracked into non-AP and non-honors tracks left high school with a 
competitive disadvantage which is implicitly tied to ethnicity. These twin effectstracking and diminished course offerings-help to explain the oft-noted overrepresentation of minority students in community colleges (Chapa \& De La Rosa, 2006).

In addition, being placed into lower academic tracks or under enrolled in college-preparatory courses can detrimentally affect achievement during a student's K-12 education. Hallinan (1994) reported that those ninth grade students sampled who were assigned to the honors track produced significantly larger, positive increments in achievement for both English and Mathematics even when background characteristics and previous achievement were controlled for. Rodriguez (2001) reported that systemic efforts to de-track students in Miami-Dade school district in combination with increased expectations for graduation resulted in a $83.7 \%$ increase in the number of minority students successfully completing math courses over a 5-year period. Indeed, discriminatory tracking in mathematics may have a decisive effect on a student's eventual success in STEM (Gross, 1993; Kahle, 1998).

The pernicious effect of tracking and under enrollment on students of limited English proficiency has also been noted. In her analysis of LEP students in Texas schools, Callahan (2005) demonstrated that tracking had a stronger, negative effect on growth in reading achievement than did a student's status as a recent immigrant, gender, or level of previous schooling. The same was true for growth in mathematics only in that case, track placement had a stronger, 
negative effect on achievement than even the student's level of English proficiency. Indeed, track placement exerted an influence on all variables measured (GPA, credits accrued, reading and math achievement) which was nearly as strong as the influence exerted by a student's English proficiency. Put simply, Callahan's analysis suggests that being placed in a lower track is as damaging to a student's academic growth as is a lack of facility with English. Finally, Callahan noted that many English language learners are not able to exit from EL programs because of a lack of growth in academic achievement, an effect exacerbated-or potentially even created-by their placement in lower academic tracks. Tracking, therefore, creates a sort of vicious cycle which, in the worst cases, serves to academically imprison some students.

The effects noted above have been hypothesized to operate through several mechanisms, primarily differences in instructional practices across tracks, differences in teacher expectations across tracks, and differences in students' motivation and self-esteem across tracks (Hallinan, 1994). Oakes (1985), for instance noted placement in lower tracks negatively affects both a student's sense of efficacy and the quality of instruction offered (Oakes, 1985). Along similar lines, Raudenbush, Rowan, and Cheong (1993) showed that higher-order skills were disproportionately taught in honors-level courses. These investigators also found that the effects of tracking on teaching approach varied by discipline noting that "the crucial results for mathematics and science were the large effects of track" (p. 543. In both STEM disciplines, students in high-track 
classes experienced a much higher exposure to high-order thinking skills than students in lower track or non-college preparatory courses.

\section{F3-4: School Size}

In the early 2000s, a great deal of attention was focused on so-called

"small school" initiatives aimed at reducing the size of high schools. This approach sought to take advantage of the intimacy of small learning communities in an effort to bolster achievement and educational opportunity for all students. William Ayers, architect and advocate of the Chicago small schools movement, wrote that "our vision of small schools was closely connected with issues of social justice, equity and community" (Ayers \& Klonsky, 2006, p. 453). Small school advocates reasoned that students who are better known by their teachers and peers are more likely to engage in their school work and remain engaged. While in some cases results have been ambiguous, researchers have, in general, concluded that school size bears an inverse relationship to student achievement (Abbott, Joireman, \& Stroh, 2002; Coladarci, 2006; CD. Howley \& Bickel, 1999; Stewart, 2009; Weis, Carolan, \& Baker-Smith, 2010).

This small school effect is most pronounced for students from underrepresented ethnic groups and less affluent families. C. Howley (1996), for example, showed important interaction effects between school size, student SES, and achievement and that those effects grew as student aged through grade level. By the time students reached their junior year of high school "the combined effects of size on achievement are moderately positive for the most 
affluent students and more strongly negative for the most impoverished" (C. Howley, 1996, p. 29). Lee and Smith (1997), reached similar conclusions, showing that the gap in mathematics achievement between low and high SES schools grew as school size grew beyond a certain threshold value and that very small schools (enrollments less than 300) performed better in this regard than very large schools (enrollments greater than 2,100 ). These authors also reported similar results for schools with high and low minority enrollments, though the effects were more pronounced. Indeed, while extremely small low SES schools performed more poorly than somewhat larger comparable schools, extremely small high minority schools outperformed their low minority enrollment counterparts. In extremely large schools, the more normal trends reappear with low-minority enrollment schools dramatically outperforming high-minority enrollment schools.

In a more recent study, McMillen (2004) showed significant and positive relationships between smaller school size and student achievement in mathematics for high school students. McMillen also showed important interaction effects between school size, student ethnicity, and parental education level. That is, small schools (those enrolling fewer than 400 students) appeared to disproportionately benefit minority students and children of parents with relatively less education. Interestingly, these same effects did not appear at a statistically significant level for elementary students, although a second, weaker impact of school size did appear. When the data were disaggregated according 
to a student's mathematics achievement in the third grade, smaller schools appeared to have served students who were below "grade level" more effectively when those students reached the fifth grade. Although this effect was small, McMillen's results raise the interesting possibility that small schools serve poor students, minority students, and students of lower academic ability more effectively.

In a comprehensive study of small schools created under the Oregon Small Schools Initiative (OSSI), investigators found that newly-formed small schools were steadily closing pre-existing achievement gaps on larger, non-OSSI schools. Students at all OSSI schools passed Oregon's math benchmark at a $25 \%$ rate during the $2004-2005$ school year compared to a $47 \%$ pass rate for all non-OSSI students. During the $2007-2008$ school year, $51 \%$ of all OSSI students passed the math benchmark, compared to $53 \%$ of all non-OSSI students, indicating that the achievement gap between OSSI and non-OSSI students was narrowing to nearly 0 . Indeed, OSSI students who qualified for free and reduced lunch actually outperformed non-OSSI students by the 2007-2008 school year, leading investigators to claim that "OSSI students perform at or above average for otherwise similar non-OSSI students in every outcome" except attendance and postsecondary enrollment (Employers for Education Excellence, 2010, p. 2).

It is relevant to note that, although school size is, in general, negatively related to important student achievement, some researchers have raised important objections. For example, Coladarci (2006) suggested that the negative 
impacts of school size may, in fact, be a statistical artifact created by the reduced sample sizes in smaller schools. Weiss et al. (2010) also suggested that "very small student groups tend to exacerbate already extant disadvantages among student groups, particularly with regard to race ... [and] it may be that larger cohort sizes provide diverse group options that may serve to mediate racial differences" (p. 173). Lastly, school size is often conflated with pedagogy, making analysis of size as an independent variable more difficult (A. Howley \& Howley, 2006). Despite these objections, however, school size will be retained in the current analysis due to its demonstrated impact on achievement for minority students.

\section{F3-5: School Choice and Governance}

Alternate school choice governance models have also been recommended as a way to positively impact student achievement. Among these, magnet schools, voucher programs, open enrollment systems, and charter schools have emerged most prevalently. Advocates of these arrangements argue that status quo school governance represents a sort of government-held monopoly on educational production which serves to inhibit the sort of fundamental organizational reform necessary (Chubb, 1988; Chubb \& Moe, 1990). Instead, schools must be granted considerable autonomy from external administrative control which, according to Chubb (1988), ranks as the "next most important source of healthy school organization" (p. 40) after parental involvement. Chubb wrote, "all other things being equal, parental environments 
are capable of shifting a school from the $30^{\text {th }}$ percentile of organizational effectiveness to the $85^{\text {th }}$, and administrative environments can boost it from the $41^{\text {st }}$ percentile to the $81^{\text {st" }}$ (p. 41). School governance models like the examples cited previously are recommended because of their shared emphasis on parental choice, parental involvement, and relatively higher levels of school autonomy.

The notion of educational vouchers was reintroduced into the national discussion by Milton Friedman in 1955 as an antidote to both the government monopoly he (and others) have argued is currently in place and to reduce the stratification in educational opportunity which operates according to which neighborhood a child happens to grow up in (Levin, 1998). Vouchers were first implemented long before Friedman, however. Voucher programs were introduced in Vermont in 1869 and again in Maine in 1873 as a way to provide education to rural students with no access to public schools (Wolf, 2010). As of 2008, approximately 70,000 students in 11 states and the District of Columbia have taken advantage of educational voucher programs. Methods for selecting voucher recipients have ranged from test of family means to evaluations of the local public school options to a child's status (or not) as a foster child. Vouchers continue to be controversial, but at least some observers contend that appropriately targeted and limited programs advance educational equity and social justice (Wolf, 2010). In contrast, Levin (1998) argued that privately controlled schools and the voucher programs that benefit them add only slightly 
to student achievement and that educational choice leads to greater racial and economic segregation of schools.

In 1990, Wisconsin launched what has become perhaps the most prominent example of voucher and parental choice programs in the United States (Belfield, 2011). Rouse (1998) reported that parental choice programs had either no effect (in the case of reading achievement) or positive effects on student achievement. Rouse also showed similar effects (positive for math, small or no difference for reading) among Milwaukee's other schools of choice, including magnet schools. In his evaluation of research generated out of Wisconsin's parental choice programs, Belfield (2011) noted that, 4 years after deployment, "researchers could find no overall positive test-score outcomes for students who participated in the program; but no negative consequences for these students either" (p. 1). In general Belfield concluded that parental choice in Wisconsin is popular in terms of parental opinion and neutral in terms of student achievement.

While voucher programs and open enrollment plans are not unknown, charter schools represent the most common alternative governance model currently deployed. As of 2004,41 states had enacted legislation allowing charter schools while during the 2002-2003 school year approximately 650,000 attended charter schools (Sass, 2006). Although a number of investigators have attempted to assess the impact of charter school implementation on student achievement independent of other factors, the results have been mixed. 
Bifulco and Ladd (2004), for example, found that students attending charter schools in North Carolina posted lower standardized test scores than students in traditional schools and that the competition created by charter schools had no statistically significant impact on test scores in nearby traditional, non-charter schools. The authors suggested that the primary reason for the lower performance in charter schools was related to their higher rates of student turnover. In their analysis of charter middle schools across 15 states, Gleason, Clark, Tuttle, Dwoyer, and Silverberg (2010) found that charter school enrollment had no statistically significant impact on academic achievement but did positively impact student and parental satisfaction with the school. These researchers did report, however, that, in some cases, charter middle schools more positively impacted the math scores of lower income and lower achieving students than did traditional middle schools.

Similarly, Sass (2006) reported lower achievement scores in Florida's charter schools which equalized with achievement scores in traditional schools after the charters had been in operation for several years. Miron and Nelson offered an excellent meta-analysis of 15 studies conducted on charter schools located in eight states (Miron \& Nelson, 2001). Like other investigators, these researchers reported strongly mixed results ranging from slightly positive effects to slightly negative effects. Importantly, these authors cautioned against premature conclusions due to the relative paucity of data and strong studies. 
Although the impact of parental choice and school governance models is still unclear, the question remains a salient one. Charter schools, magnet schools and open enrollment plans are at the forefront of school improvement efforts. The passage in Oregon of HB 3681 in 2012 (Oregon Department of Education, 2011a) constitutes one important case in point. This legislation allows students to transfer across district boundaries without permission from their home districts, a bill lauded by some as a move toward natural competition and school improvement but opposed by others as deleterious to equity and opportunity in schools serving minority and less affluent populations. Right or wrong, schools of choice will continue to be opened (and closed) across the country, recommending school choice and governance as important predictor variables within the proposed analysis.

\section{F3-6: Academic Momentum}

The concept of academic momentum received wide attention through the work of Adelman (2006) who investigated the effect on a student's patterns of course taking on their eventual success in attaining a bachelor's degree. Adelman concluded that patterns of course taking in high school were strongly predictive of a student's likelihood to finish an undergraduate degree. In his examination of trends in AP participation in California schools, Campbell (2007) included an academic momentum construct which is somewhat congruent with Adelman's work. For Campbell, however, academic momentum was measured as improvement in proficiency rates on state assessment tests and not in terms 
of patterns of course taking or the grades received in those courses. Campbell also found that academic momentum was positively related to academic outcomes.

Attewell, Heil, and Reisel (2011) noted that Adelman's earlier definition of academic momentum suffered from being too-broadly defined and was "susceptible to problems of causal circularity" (p. 28). Attewell proposed a fourcomponent model of academic momentum comprised of a student's delay between high school graduation and matriculation into a 2- or 4-year college, the number of credits a student takes during his or her first year, the "high credit" status of a student (coded as 0 or 1 ) which captures whether or not a student enrolls in a large number of credits during his or her first term, and "summer attendance" status of a student (coded 0 or 1 ) which captures whether or not a student enrolls in summer school during his or her first summer. Delayed enrollment and enrolling part time during the first term were negatively associated with the attainment of a bachelor's degree while attending during the first summer was positively associated with the attainment of a bachelor's degree. The impact was shown to exist for students who enrolled in a large number of credits early in their collegiate careers.

Several researchers (W. Hoy, Tarter, \& Bliss, 1990; W. Hoy, Tarter, \& Hoy, 2006; Lee \& Bryk, 1989) identified academic focus as an important predictor of school climate and academic achievement. W. Hoy et al. (2006) developed a construct called "academic optimism" constituted by measures of staff collective 
efficacy, faculty trust of students and parents, and academic emphasis of a school, sometimes called "academic press." Academic optimism had a statistically significant and positive impact on student achievement roughly equal in magnitude to the effect of SES as an independent predictor and stronger (and in the opposite direction) than the effect of urbanicity.

This investigation focused around identifying factors the positively impact minority success in AP Calculus. Data included measures of AP participation and AP success (score distributions) on a school level, data which address the third of Hoy's components, academic emphasis. Academic emphasis was more specifically defined as being "descriptive of the normative and behavioral environment of the school at both the classroom and school level" (McGuigan \& Hoy, 2007, p. 205). Academic emphasis has been shown to positively impact student achievement in urban elementary schools (Goddard, Sweetland, \& Hoy, 2000), high-poverty middle schools (Picucci, Brownson, Kahlert, \& Sobel, 2002), and high schools (Craig et al., 2001; W. Hoy et al., 2006; Wagner \& DiPaola, 2011). These investigators found that academic emphasis was an important predictor of student success when controlling for SES and, in some cases, was as important as SES in the prediction of standardized measures of academic achievement (A. Hoy, 2012).

\section{Summary}

Six school-level factors have been recommended for inclusion as predictors of minority participation and achievement in AP Calculus: School-level 
expenditures including teacher/student ratios, teacher qualifications, rates of participation in college-preparatory courses and tracking, school size, school governance, and academic momentum. Each of these have been advocated as important predictors of student achievement, although the direction and size of impact is, in most cases, under debate. Due to their salience within the discussion of educational improvement, all six are recommended for inclusion in the current analysis. The operationalization of these concepts will be discussed in the methodology section. In addition, two other school-level variables (schoolaggregated SES, size of the ethnic groups) will be recommended, described and discussed.

\section{A Spotlight on AP Calculus Achievement}

Before describing the sampling and analysis methodology, it is necessary to more fully establish the trends of under participation and achievement for minority students in AP Calculus. First, on a national scale it is readily apparent that Black and Hispanic students are dramatically underrepresented. In 2007, approximately 204,000 AP Calculus exams were administered in the United States. Approximately 133,000 of them-or about $65 \%$-of those exams were taken by students who self-identified their ethnicity as non-Hispanic White. During that same year, 9,329 AP Calculus tests were administered to students who identified themselves as Black and 8,715 exams administered to students who identified themselves as Mexican American. Another 7,607 were administered to students who identified themselves as other Hispanic and 1,007 
were administered to students who identified themselves as Puerto Rican. In total, 26,658 AP Calculus exams were administered to non-Asian minority students while 31,922 exams were administered to Asian students. As a proportion, $22 \%$ and $26 \%$ of all AP exams administered in 2007 were taken by non-Asian and Asian minority students respectively (The College Board, 2007). An analysis of population estimates from the United States Census Bureau (2012) shows that $34 \%$ of all $15-19$ year olds were Black or Hispanic while $4 \%$ were Asian. A comparison of these rates of representation to the take rates for Asians, Blacks, and Hispanics shows a clear trend of over representation for Asians and underrepresentation for Blacks and Hispanics

Similar trends emerge from the 2010 census and AP Calculus data. In that year, $15 \%$ of all AP Calculus exams were taken by Black and Hispanic students while $16 \%$ of all AP Calculus exams were taken by Asian students. These rates of participation compare unfavorably to the rates of representation in the population for 15-19 year olds in the case of Blacks and Hispanics (38\%) but favorably to the rates of representation for Asian teenagers (4\%). These numbers equate to a nearly $39 \%$ rate of underrepresentation for Blacks and Hispanics and a $400 \%$ rate of over participation for Asians. It is important to note that categories of minorities differ between organizations, that ethnicities are self-reported, and that the population values represent estimates based on the 2000 decennial census. Comparisons of this sort must therefore be interpreted with caution. It is 
clear, however, that Hispanic and Black students have historically been and continue to under participate in AP Calculus.

Similarly divergent trends are observed in average scores obtained on the AP Calculus exam. In 2007, all test takers calculated together registered an average score of 2.92 on a 5-point scale. The distribution of scores was roughly bimodal, with the $46 \%$ of test takers registering either a 5 or a 1 . A somewhat more uniform score distribution emerged for White students, with nearly equal numbers of test takers registering scores at each level. That same cannot be claimed for Black and Hispanic students. Of the 26,658 Black and Hispanic students who took the AP Calculus exam in $2007,13,610$ of them (51\%) registered the lowest possible score, while fewer than $10 \%$ registered the highest possible score. Non-Asian minority students recorded approximately $25 \%$ of all 1 's and just $5 \%$ of all 5 's. Similar trends emerged in 2010. Black and Hispanic students recorded $29 \%$ of all 1 's and $6 \%$ of all 5 's, showing, once again, a clear deficit not only in participation but also in achievement. Figure 3 shows these results in histogram form for 2011 data. 


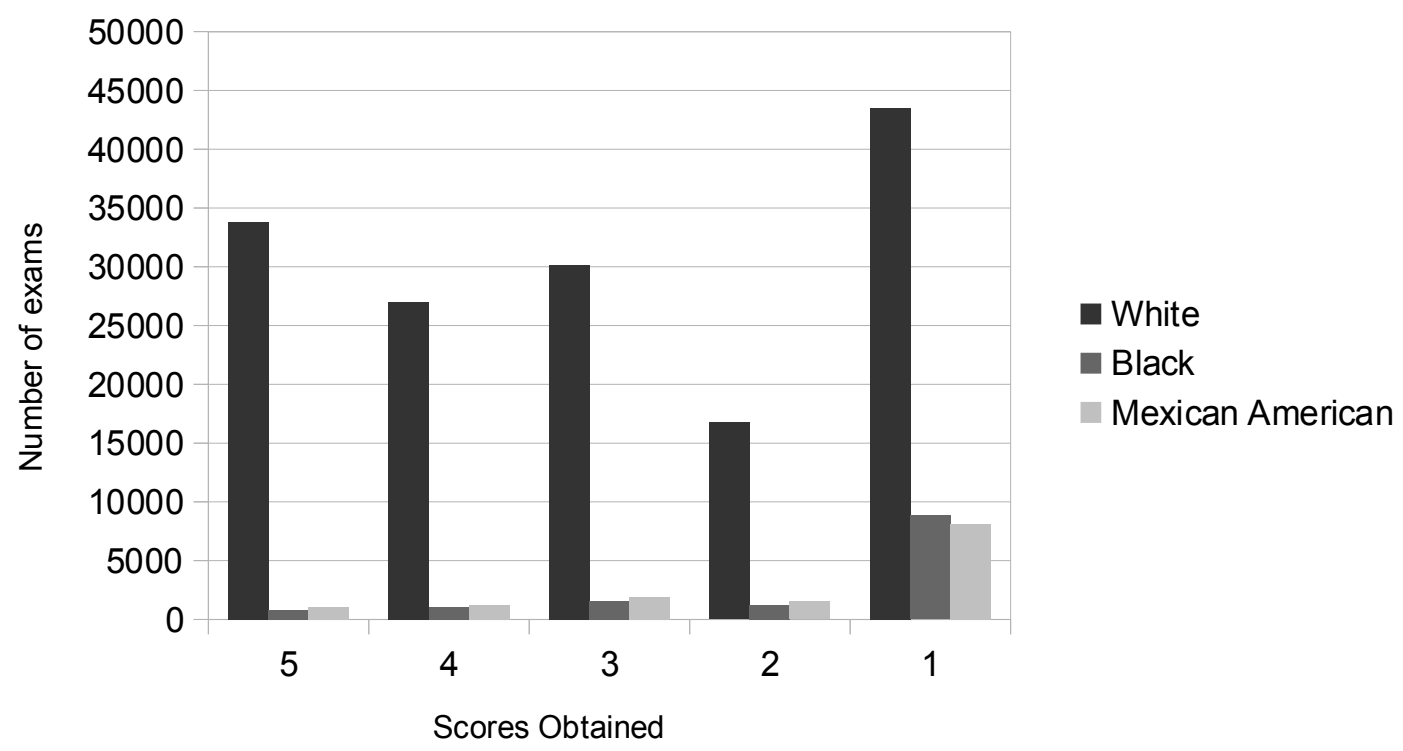

Aggregated score distributions on all exams for selected populations, 2011. Results show a clear difference in distribution according to ethnicity.

Figure 3. AP Calculus score distributions for selected groups, 2011.

Patterns of under achievement for Black and Hispanic students are also evident in state-level data, although distinct inter-state differences exist. Figure 4 shows a simple bar graph of score distributions for Black and Hispanic students on AP Calculus in 2011 for states selected for having relatively large numbers of passing scores posted by Black and Hispanic students. Oregon is also included for purposes of comparison. It is immediately apparent that the number of exams varies widely by state and that, to some lesser degree, the score distributions also vary by state. 


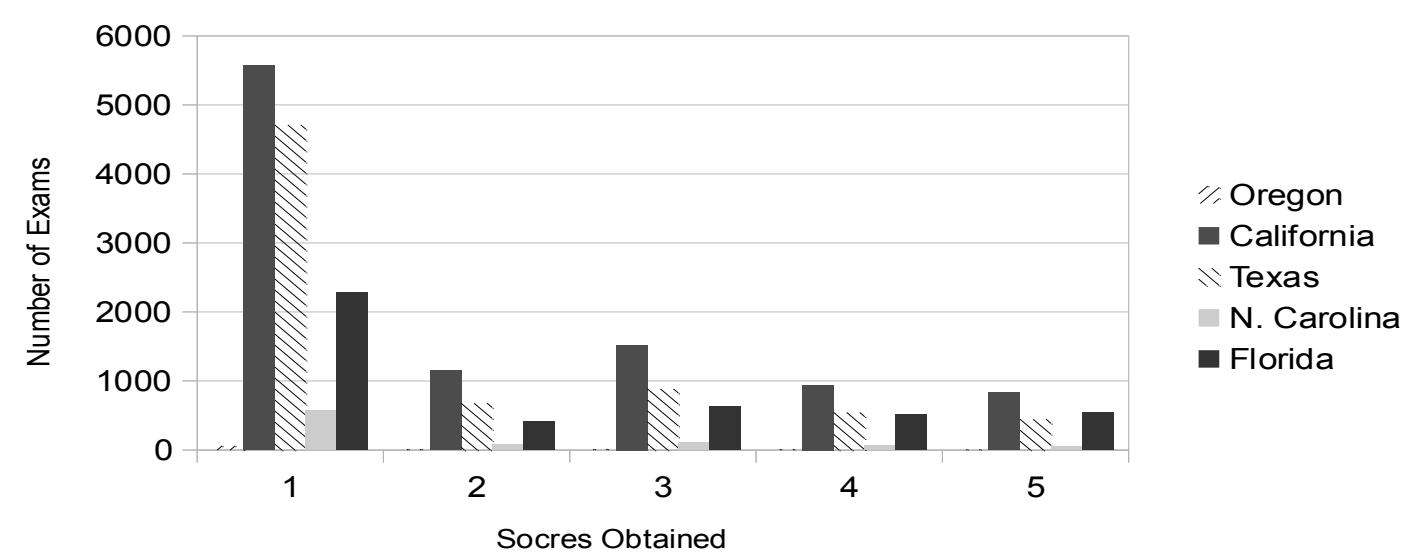

Score distributions posted by Black and Hispanic students for selected states, 2011. Results show clear between-state differences.

Figure 4. AP Calculus score distributions for minority student, selected states.

Figure 5 offers an alternative way to view differences in score distributions. In this case, the $y$ axis represents the fractional proportion of scores at each score level for selected states. In all cases, well over half of all non-Asian minority students score 1s. However, the fraction proportion of students scoring at higher levels is visibly larger in California and Florida. An exploratory chisquared analysis of proportions of Black students scoring three or better shows that these interstate differences are indeed statistically significant at $p<0.005$, suggesting that state-level factors might be playing an important role in encouraging higher AP Calculus scores by Black and Hispanic students. These results must be interpreted with caution, however, because sample sizes are extremely small in some cases (in Oregon, for example), so inter-state differences, though statistically significant, may not be the result of state-level 
factors. Because of the small sample sizes, it may be possible that the largest fraction of AP test takers come from one or two high schools. In this case, observed inter-state differences may in fact be the result of inter-school differences or inter-district differences.

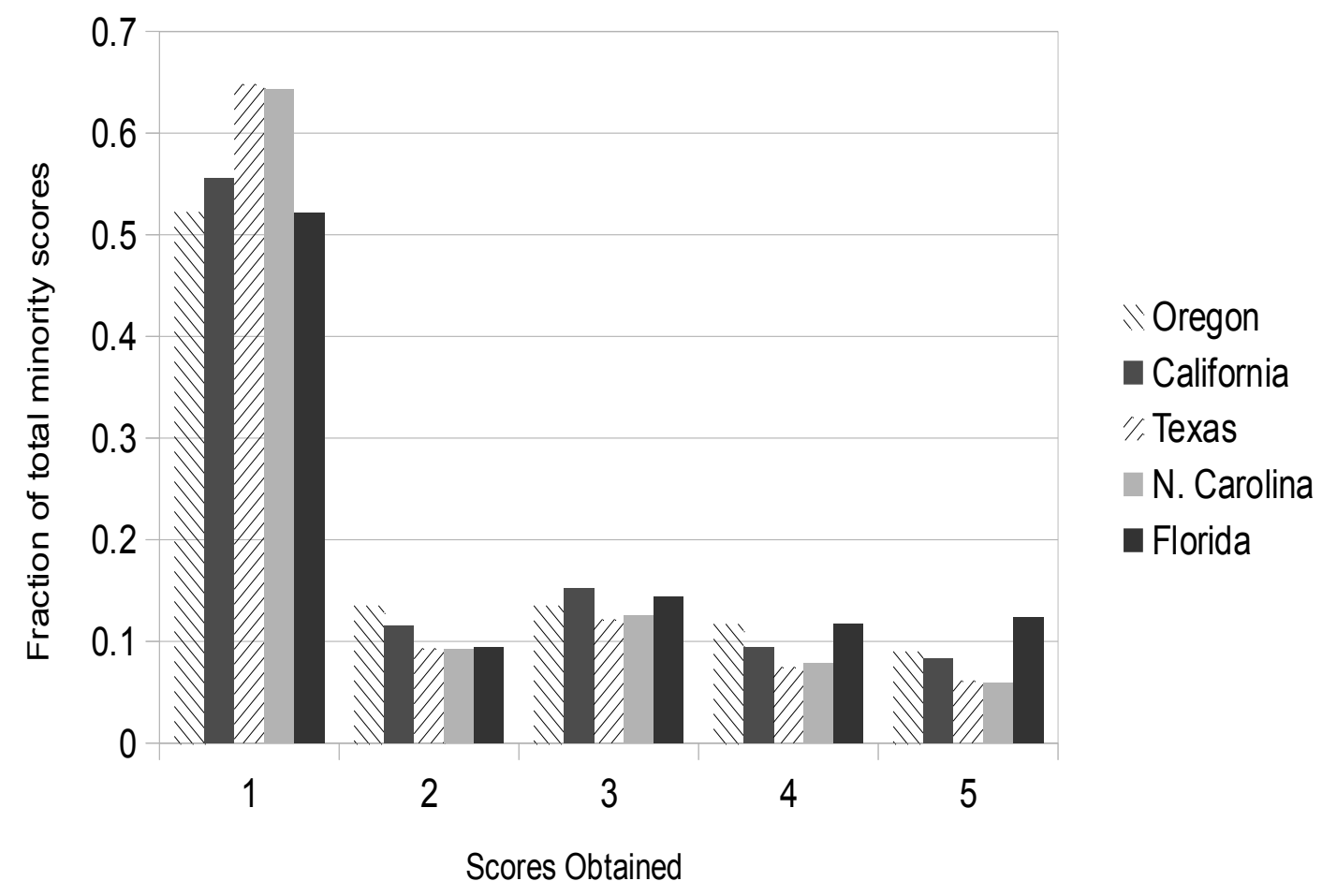

Distribution of passing scores (scores of 3 or better) posted by Black or Hispanic students disaggregated by state.

Figure 5. Proportion of AP Calculus test takers scoring 3 or better, minority students, selected states.

The current proposal seeks to identify school-level factors which impact the growth in participation and achievement of Black and Hispanic students in AP 
Calculus. From the exploratory analysis offered here, it is clear that AP Calculus participation and achievement varies over time and between states. It is also apparent by the theoretical considerations offered previously that at least some school-level factors are likely contributors to variation across schools and across time. The following section operationalizes the key variables for investigation, describes the research design and methodology for selecting participating school districts, and outlines the method of analysis identified for use in this investigation. 


\section{CHAPTER 3}

\section{METHODOLOGY}

This section describes the data collection and analysis methodology utilized in this investigation. In cooperation with 10 large, urban school districts, a large and geographically distributed database was developed which included school-level score distributions on AP exams and several other school-level factors. A combination of conditional and unconditional linear growth models and multi-variate regressions were used to elucidate critical factors contributing minority success in AP Calculus. This chapter begins by describing the sample selection and data collection processes and concludes with a discussion of model specification and analysis techniques.

\section{Selection of Sample and Data Collection}

Five criteria were used in selecting school districts as potential study participants. First, in order to simultaneously maintain student confidentiality and statistical relevance, only those school districts which served large, diverse populations were considered. Second, school districts which were widely distributed geographically were approached in order to increase the generalizability of results. Third, because this investigation focuses on identifying school-level factors which contribute to the success of minority students in AP Calculus, only those districts with a demonstrated commitment to college-ready programs like AP were considered. Fourth, large school districts were considered 
desirable because the statistical reliability of the results improves as the number of high schools and students included in the sample increases. Finally, those school districts in which a wide range of school-governance models (small schools, charter, magnet, etc.) are deployed were considered desirable in order that school governance could be included as a predictor in the analysis. The member districts of the Council of Great City Schools (n.d.) fit these criteria and were identified as the pool of potential study participants.

By policy, The College Board requires written permission from each participating school district in order for outside researchers to obtain school-level score distributions. Ten districts in seven states agreed to participate in the study. School-level AP exam score distributions were provided in digital format by The College Board for 388 schools serving approximately 290,000 students across 5 years in the 10 participating districts. In all cases, score distributions were disaggregated by subject and by ethnicity. AP exam score distributions were not disaggregated by SES. No individual students were identifiable in the datasets provided and schools were coded by the author in order to maintain school-level confidentiality. Additional school-level variables were obtained from publicly available websites in each state and school district. Some data were also downloaded from the National Center for Educational Statistics. Data availability varied widely from district to district. A complete inventory of data and sources is available in the Appendix. By request of the participating school districts, district codes are used to maintain district-level confidentiality. 


\section{Data Processing and Data Equivalence}

Data processing proceeded in three levels. First, the raw, unprocessed data were obtained from digital files provided by The College Board or downloaded from public web sites (see Appendix). Formats varied widely from source to source. Next, data values of interest were extracted from the raw data files using scripts developed in Perl. In all cases, data values were extracted only and were not changed from their raw form. In one school district, digital files were not available and so data values of interest were hand entered into an Excel-style spreadsheet. Finally, the semi-raw data were transformed into flat text files in uniform formats in order to provide for ease of use. In some cases, data values were transformed into standard forms for cross compatibility.

Disaggregated enrollments were reported as total numbers of students in each sub group. In one school district, enrollments by ethnicity were provided as percentages of the total enrollment. These values were transformed into total numbers for each sub group by simple multiplication.

Scores on state assessments also varied widely in meaning. In 8 of the 10 school districts, raw data were reported as percentages of each subgroup scoring proficient or above. For one of the school districts, math proficiency was measured by one or more end-of-course (EOC) high school exams. In this case, the number of exams passed on all mathematics EOC exams were summed and then divided by the total number of those tests administered in order to obtain a percentage who scored proficiency. In one case, a single percentage proficiency 
number was reported for all EOC exams, including tests of mathematics, reading, and language arts. In this case, the scores were reported as English Language Arts scores. In some cases, reading scores were reported. In other cases, English language arts scores were reported. These were taken as equivalent scores and labeled as "reading language arts."

Staffing and fiscal data availability varied widely in terms of coverage and variables reported. The National Center for Educational Statistics (NCES) provides total teacher FTE at a teacher level, so that data was available for all schools. Only two districts provided ready and public access to fiscal data, and these data were disaggregated along somewhat different lines. Only two school resource variables were used in the analysis-teacher FTE and total building-level expenditures. Both of these variables, where available, were defined in a consistent manner across districts.

At each level of data extraction and processing, the resulting files were systematically checked for consistency and accuracy against the previous processing level. Downloaded raw data were cross checked with equivalent sources on the various web sites. Level one data were cross checked with raw, downloaded data for accuracy. Level two data were cross checked with level one data for accuracy of extraction and, in some limited cases, accuracy of computations. One example of the latter was the conversion of percentages of students in a specific sub group to total numbers of students. Data obtained from The College Board were subject to an external quality assurance procedure and 
were assumed to be complete and accurate. Computations required to obtain all non-primary, derived variables were completed in a final, fourth level of processing prior to analysis. The derivations of these derived variables are described in the next section.

\section{Operationalization of Variables}

Five school-level factors were previously identified for inclusion in the analysis: school-level funding, differential participation in college-preparatory courses, teacher quality, school size, and school governance. School governance was ultimately discarded as a variable for the current analysis due to the wide variations in implementation of charter schools, magnet schools, schools of choice, and schools subject to open enrollment. In order to be measured, each of the remaining variables were given clear operational definitions which, ideally, possessed face validity, construct validity, content validity, and predictive validity (Evans \& Rooney, 2008). Identifying the predictive validity of these factors with respect to student achievement in AP Calculus is, in fact, the goal of this investigation. Therefore, only face validity, content validity, and construct validity are discussed. Operational definitions are further limited by data availability. While in some cases, bulk, school-level variables are readily available, in other cases, a compromise was made between data access and validity. In each case, a clear definition is offered and measurement validity is addressed. 
F3-1 School funding: Aggregated school level funding was publicly available for 2 of the 10 districts included in the sample. For the purposes of analysis, total aggregated expenditures at the school level were utilized without respect to how those expenditures were allocated. This definition possesses strong face validity (reasonable on the face of it) and construct validity (measures the underlying construct) but has questionable content validity. That is, while total school-aggregated expenditures may have predictive value for AP Calculus success, it is possible that between school differences in fund allocation would also be important. While a full reckoning of inter school differences in fund allocation would be impractical to obtain, teacher-student ratio forms a reasonable proxy. In addition, teacher-student ratio is recommended on theoretical and empirical bases to be an important school-level predictor in its own right (Engberg \& Wolniak, 2010; Perna, 2006). Since this variable is also either readily available or readily calculable from publicly available data, it will be included in the operational definition of school funding. In the analysis, teacherstudent ratio and total, building-level expenditures were normalized to the total enrollment of the school for the sake of consistency across schools. The computation of these variables is described in the proceeding section.

F3-2 Teacher quality: Teacher quality is difficult to operationalize or measure in a way that permits for a large-scale analysis. Years of experience, level of educational degree obtained, and type of teaching credential obtained are common proxies for teacher quality within existing literature as discussed in 
the previous chapter. While some investigators have shown that these measures are positively correlated with student achievement, others, such as Chubb (1988) have questioned the validity of this construct. Teacher quality doubtless also includes parameters related to classroom, professional practice, pre-service training, teacher standardized test scores, and others that are much more difficult to measure in a bulk, longitudinal dataset (Akiba, LeTendre, \& Scribner, 2009; Goe, 2007; A. Harris \& Sass, 2008). Average number of years of experience for teachers was available from 6 of the 10 school districts and percentage of teachers with at least a master's degree was available from 4 of the 10 school districts. These two variables were included within the time-averaged regression analysis described in the proceeding chapter.

F3-3 Participation in college preparatory courses: The literature review in chapter 2 showed the importance of rigor during a student's high school years and the relative underrepresentation of minority students in college preparatory courses. In the current investigation, participation in AP Calculus forms one of the primary dependent variables. Expanded to include participation in all AP subjects, this measure forms a reasonable proxy for enrollment in collegepreparatory courses. Like the previous operational definitions, this definition suffers from some lack of construct validity (students enroll in other sorts of college-preparatory courses in addition to AP courses), but longitudinal, schoollevel enrollment data are not readily available. As with staffing and expenditure data, rates of passing and taking AP exams are normalized to the population of 
the sub group being analyzed. For example, the rate of production of passing scores on AP Calculus posted by minority students was computed by dividing the number of passing scores in a specific school by the number of Black and Hispanic students enrolled in that school. Similarly, the rate of production of passing scores on non-Calculus AP exams posted by the total population was computed by dividing the number of passing scores in a specific school by the total number of students enrolled in that school.

F3-4 School size: School size is operationalized as total enrollment without respect to school type. Where schools-within-a-school exist, only the size of the sub school or academy is utilized. This measure possesses face validity, construct validity, and content validity due to the simplicity of the construct.

\section{School-Aggregated SES and Ethnic Composition}

School-aggregated measures of SES are routinely included as important variables in school effects studies on student achievement (Borman \& Dowley, 2010; Rumberger \& Palardy, 2005). Engberg and Wolniak (2010), for instance, showed that school-aggregated SES had a highly significant and strong impact on college enrollment while Ma and Klinger (2000) showed that schoolaggregated SES had statistically significant impacts on mathematics, reading and writing achievement for sixth grade students. Recent work by Reardon, Valentino, and Shores (2012) underlined the importance of family income as a predictor of academic achievement. These investigators show that low-income eighth graders today are 5 years behind high-income eighth graders in terms of 
knowledge-based literacy skills. Additionally, the "income achievement gap" has grown considerably over time and "is now a better predictor of children's success in school than race" (Reardon, 2013, para. 6). These results strongly recommend the inclusion of SES and point toward an important area for additional investigation. In the current investigation, the proportion of students identified by the school district as economically disadvantaged is utilized as a metric for school-level SES.

Ethnic composition of a school's student body has also been cited as a possible predictor of student achievement, most notably by the Coleman Report of 1966 (Coleman et al., 1966). Indeed, the authors of this widely cited, influential, and controversial paper suggested that a school's social and ethnic makeup were more influential to student achievement than any other school factor or, for that matter, the student's own background characteristics (Coleman et al., 1966; Rumberger \& Palardy, 2005). These arguments gain some theoretical foothold in the work of Allport, Ogbu, and Steele previously cited, since the formation of out groups and oppositional responses are socially constructed phenomena. A school that is composed of a traditionally underserved minority population, for example, might struggle to escape stereotype threats associated with academic achievement. This finding was strongly corroborated by Borman and Dowley (2010) who suggested that attending a "high-poverty school or a highly segregated African American school has a profound effect on a student's achievement outcomes, above and beyond 
the effect of his or her individual poverty or minority status" (p. 1239). For this analysis, ethnic composition will be operationalized as the proportion of the total student body identified as either Black or Hispanic. This is an over generalization with respect to ethnic heritage and creates, once again, difficulty with construct validity. This limitation is largely inevitable, however, since ethnicity is selfreported and the ethnic categories are developed by those collecting the data. Where variation between these two ethnic categories are reported (e.g., Mexican American and "other Hispanic"), results will be aggregated appropriately.

\section{Academic Momentum}

The author's personal experience with AP coursework recommends for inclusion one final school-predictor: Academic momentum. In 2005, Corbett High School in Corbett, Oregon began to aggressively pursue an "AP for all" policy in which virtually every student took one or more AP courses. Somewhat contrary to expectations, as the rate of participation went up, so did the proportion of students earning 3's or better on various end of year AP exams. Steve Fisk, Assistant Principal at North Central High School in Spokane, Washington reports corroborating results, an especially important result given that relatively diverse population North Central High School serves (Fisk, personal communication, March 20, 2013). These observations coupled with the theoretical considerations presented in chapter 2 recommend the inclusion of a measure of academic momentum. 
For this analysis, academic momentum was constituted by three measures: improvements in rates of proficiency on state standardized measures of math achievement at the high school level, improvements in rates of proficiency on state standardized measures of reading language arts achievement at the high school level, and changes in total proportion of "passing" scores (scores of 3 or more) across all subjects. This metric, which is consistent with Campbell's (2007) conceptualization of academic achievement as a change process, is related to Hoy's academic emphasis. It includes rates of success on AP exams which are naturally related to a school's commitment to scheduling AP courses and administering AP exams. However, academic momentum captures the rate of change in these values and includes measures of success on state assessments, and so it constitutes a different predictor. Hence this study also explores rates of success on non-Calculus AP exams as important predictors of minority success in AP Calculus.

\section{Computation of Derived Variables}

The methods of computation for the derived quantities included in the analysis are described in Table 1. In all cases, the subscript i refers to school and the subscript t refers to time level. 
Table 1

Definition of Derived Variables

Production rate of AP Calculus scores for minority students

MinorityCalcPrd $_{i, t}=\left(\left(\right.\right.$ blackCALCsch $_{i, t}+$ hispanicCALCsch $\left._{i, t}\right) /$ MinorityENRsch $\left._{i, t}\right) * 1000$

Production rate of AP exam (non-Calculus) scores for total population

TotAPPRD $_{i, t}=\left(\left(\right.\right.$ TotAPsch $_{i, t}-$ TotCALCsch $\left._{i, t}\right) /$ TotENRsch $\left._{i, t}\right) * 1000$

Proportion of minority (Black and Hispanic) enrollment

MinorityRATIO $_{\mathrm{i}, \mathrm{t}}=\left(\right.$ blackENRsch $_{\mathrm{i}, \mathrm{t}}+$ hispanicENRsch $\left._{\mathrm{i}, \mathrm{t}}\right) /$ totENRsch $_{\mathrm{i}, \mathrm{t}}$

Proportion of economically disadvantaged enrollment

EconRATIO $_{\mathrm{i}, \mathrm{t}}=$ econENRsch $_{\mathrm{i}, \mathrm{t}}$ TotENRsch $_{\mathrm{i}, \mathrm{t}}$

Per pupil teacher FTE

PerPupilFTE $\mathrm{E}_{\mathrm{i}, \mathrm{t}}=$ staffFTEsch $_{\mathrm{i}, \mathrm{t}}$ totENRsch $_{\mathrm{i}, \mathrm{t}}$

Per pupil total school-level expenditures

PerPupilTOT ${ }_{i, j}=$ expendTOTsch $_{i, t} /$ totENRsch $_{i, t}$

Academic momentum for total population

TotACMMT $_{i, j}=\left(\right.$ totMTHsch $_{i, t}-$ totMTHsch $\left._{i,-1}\right)+\left(\right.$ totRDGsch $_{i, t}-$ totRDGsch $\left._{i,-1-1}\right)+\left(\operatorname{totAPPRD}_{i, t}-\right.$ totAPPRD $\left.D_{i, j-1}\right)$

The above derived variables and the non-derived, raw data values were transformed into time-averaged quantities by summing the values available at each time level and dividing by the number of real data values available according to: 
Time-averaged quantity $\mathrm{i}_{\mathrm{i}, 0}=\left(\mathrm{S}\right.$ Quantity $\left._{\mathrm{i}, \mathrm{t}}\right) /($ Number of available values $)$

Note that missing values did not contribute to the time-averaged quantities.

\section{Analysis of Variation Over Time}

One key contention implicit in the previous definition of academic momentum is that minority achievement in AP Calculus changes over time and that this rate of change is malleable under the influence of school level factors. In order to assess the validity of this claim, two things must be in place. First, longitudinal data must be available which track the variables of interest over time at a school level. This requirement was built into the data requests and data collection procedures. The longitudinal, panel dataset developed therefore allows for this type of analysis. Second, an analysis technique must be utilized which allows for school-level covariates to impact rates of change of the outcome variable over time.

A multi-level, individual growth model is able to accommodate this type of analysis. For the current analysis, the level one equation describes the variation of the outcome variable over time and the level two equations describe the effects of the school-level covariates. Following the approach of Singer (1998), the level one equation is:

$$
Y_{i j}=P_{0 j}+P_{1 j}\left(T_{i m e} i j\right)+r_{i j}
$$

In this case, the subscripts $i$ and $j$ refer to time and school respectively. $Y_{i j}$ is the outcome variable for school $\mathrm{j}$ at time $\mathrm{i}$ and $\mathrm{r}_{\mathrm{ij}}$ describes the unaccounted for 
variation in the outcome variable. The coefficients $P_{0 j}$ and $P_{1 j}$ describe the value of the outcome variable at time level 0 for school $\mathrm{j}$ and the slope of the outcome variable over time for school $\mathrm{j}$ respectively. The level two equations for an unconditional individual growth model are as follows:

$$
\begin{aligned}
& P_{0 j}=b_{00}+m_{0 j} \\
& P_{0 j}=b_{01}+m_{1 j}
\end{aligned}
$$

In this case, no level two covariates are included. The coefficients $m_{0 j}$ and $m_{1 j}$ describe the level two (between school) variations in intercept and slopes. The variances of these quantities allow the analyst to determine whether level two covariates might have an impact on initial conditions and rates of change over time of the outcome variable. Where the variances are significant, level two covariates can be included to account for differences in slopes and intercepts. A set of equations including time-averaged math achievement as a single, schoollevel covariate are shown here.

$$
\begin{gathered}
\text { Level one equation: } \\
\mathrm{Y}_{\mathrm{ij}}=\mathrm{P}_{0 \mathrm{j}}+\mathrm{P}_{1 \mathrm{j}}\left(\text { Time }_{\mathrm{ij}}\right)+\mathrm{r}_{\mathrm{ij}} \\
\text { Level two equations: } \\
\mathrm{P}_{0 \mathrm{j}}=\mathrm{b}_{00}+\mathrm{b}_{01}\left(\text { TotMathAve }_{\mathrm{j}}\right)+\mathrm{m}_{0 \mathrm{j}} \\
\mathrm{P}_{1 \mathrm{j}}=\mathrm{b}_{10}+\mathrm{b}_{11}\left(\text { TotMathAve }_{\mathrm{j}}\right)+\mathrm{m}_{1 \mathrm{j}}
\end{gathered}
$$

Within a mixed models framework, these equations are combined into a single equation: 


$$
\begin{gathered}
Y_{i j}=\left[b_{00}+b_{01}\left(\text { TotMathAve }_{j}\right)+b_{10}\left(\text { Time }_{i j}\right)+b_{11}\left(\text { TotMathAve }_{j}\right)\left(\text { Time }_{i j}\right)\right]+\left[m_{0 j}+\right. \\
\left.m_{1 j}\left(\text { Time }_{i j}\right)+r_{i j}\right]
\end{gathered}
$$

The terms in the first set of brackets describe the fixed, school-level

effects. $b_{00}$ describes the value of the outcome variable when both time and timeaveraged math achievement are zero. $b_{01}$ and $b_{01}$ describe the effect of timeaveraged math achievement and time on the outcome variable respectively. $b_{01}$ captures the interaction effect between time and time-averaged math achievement. When this coefficient is non-zero, time-averaged math achievement can be said to have an effect on the rate of change over time of the outcome variable. The terms in the second set of brackets describe the random, within-school and between-school variations in the outcome variable.

\section{Analysis of Time-Averaged Quantities}

Results from the mixed model analyses showed that time and the interaction effects between time and time-averaged covariates were, in most cases, not significant predictors of minority success in AP Calculus. In contrast, a number of time-averaged covariates did prove to be significant predictors. That is, while variations in the outcome variable over time were not significant and were not significantly impacted by school-level factors like total enrollment, a number of school-level factors did emerge has having import predictive power with respect to the outcome variables. In order to more directly assess the impacts of these factors, a set of multi-variate, linear regressions were performed in a backwards, stepwise fashion. The fully-specified model began with the 
complete set of variables just described. The governing equation for this model is:

$$
\mathrm{Y}_{\mathrm{j}}=\mathrm{b}_{0}+\mathrm{b}_{1}{ }^{*} \mathrm{~F} 1^{\mathrm{j}}+\mathrm{b}_{2}{ }^{*} \mathrm{~F} 2_{\mathrm{j}}+\mathrm{b}_{3}{ }^{*} \mathrm{~F} 3_{\mathrm{j}}+\ldots+\mathrm{b}_{11}{ }^{*} \mathrm{~F} 10_{\mathrm{j}}+\mathrm{r}_{\mathrm{j}}
$$

In the equation above, the outcome variable $Y_{j}$ is the rate of production of passing scores on AP Calculus exams posted by minority students. The meaning of the other time-averaged factors F1 through F10 are described in the Table 2. The rationale behind the selection of these variables is described in the next section. Note that these are the same time-averaged factors used as school-level covariates in the individual growth models describe earlier.

\section{Table 2}

\section{Description of Time-Averaged Variables}

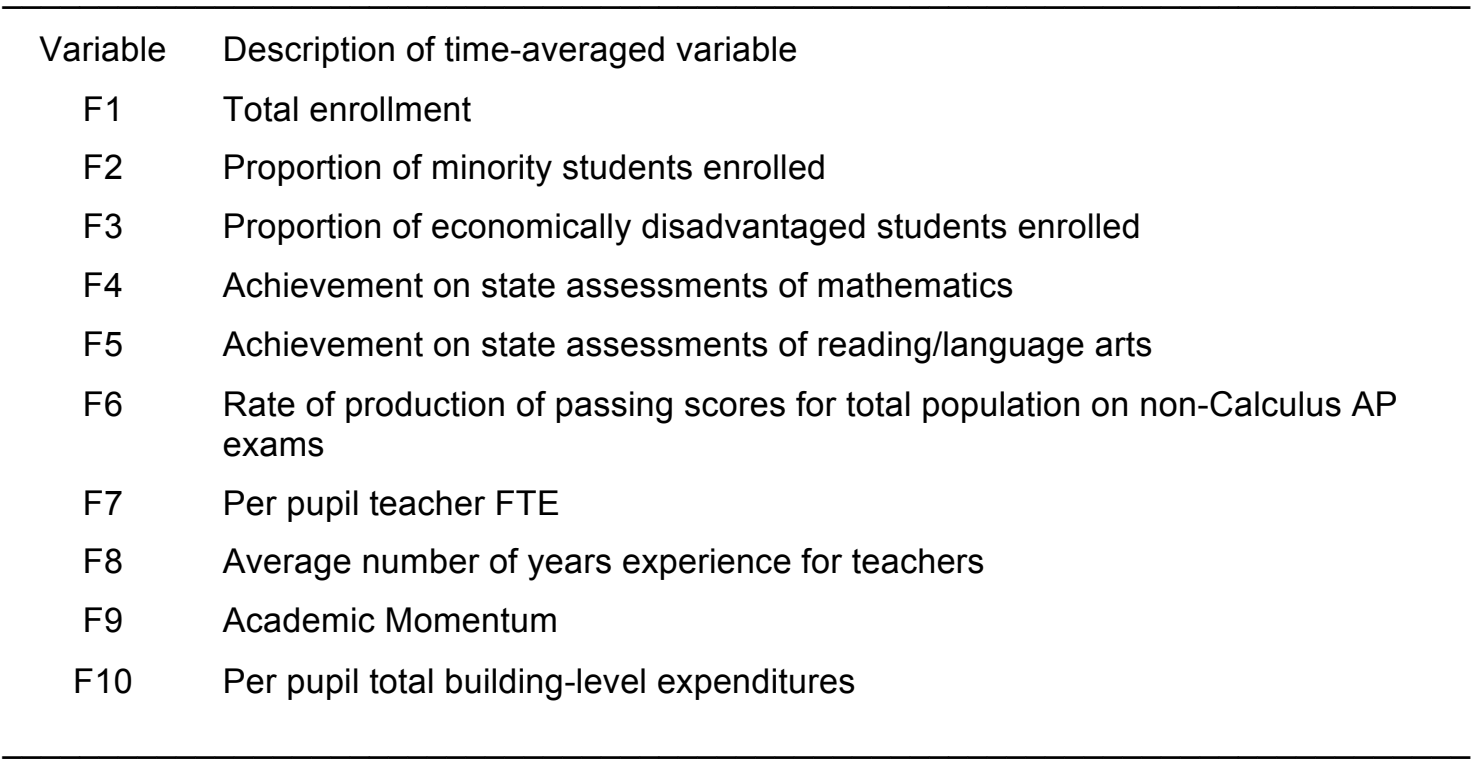

Because data coverage varied widely from district to district, four discrete datasets were developed and multi-variate regressions were run on these 
datasets separately. In each case, variables were eliminated from the fullyspecified model according to computed $p$-value. The variable with the highest $p$-value was eliminated and the model was run again. Final model selection was based on the adjusted $R$ squared value in order to account for the higher level of fit associated with more complicated models. Results are detailed in the next section.

\section{Selection of Variables for Modeling}

The full dataset is quite large with respect to some variables. College Board codes were provided for 388 schools. Disaggregated enrollments were available for 361 schools across 5 years. Disaggregated achievement levels for state assessments in mathematics were available for 278 schools across 5 years. The dataset is considerably sparser, however, with respect to other important variables; 158 of the 361 schools reporting disaggregated enrollment values produced non-zero values for minority AP Calculus production. Of those 158 schools, 133 also reported non-zero values for time-averaged variables F1 through F7 (see Table 2 for variable definition). The choice of variables to include in the model therefore constituted a compromise between including important variables and contending with missing data.

The majority of schools in the sample reported no production of passing scores on AP Calculus for minority (Black and Hispanic) students. Considering these two populations separately further reduced the available sample size, particularly with respect to Black students. In order to address the questions of 
interest and to maximize the number of data points available for analysis, Black and Hispanic students were considered together as a single category. This choice further constrained the development of the other variables for analysis.

Evidence cited in the preceding literature review supports the inclusion of variables relating to the demographic composition of the school (e.g., Callahan, 2005; Chapman et al., 2010; S. Lewis et al., 2011;Oakes, 1985), the total enrollment of the school (Abbott et al., 2002; Coladarci, 2006; C. Howley \& Bickel, 1999; Stewart, 2009; Weis et al., 2010), teacher quality and school-level resources (Condron \& Roscigno, 2003; Croninger et al., 2007; Ingersoll, 2002), and prior achievement in math and reading (Mattern et al., 2009; Sadler \& Tai, 2007). School-level data were available in all of these categories, though to varying degrees of coverage. Total enrollment (school size) was available for all schools which also reported non-zero production of minority AP Calculus. Achievement data on state assessments of math and reading were available in virtually all cases. However, these data were reported as percentages of total test takers who were rated as proficient or above disaggregated by ethnicity. Reported this way, rates of proficiency for Black students could not be added to rates of proficiency for Hispanic students to obtain rates of proficiency for an aggregated minority value. Therefore, rates of proficiency for the total population were utilized.

Teacher quality data were sparsely available. Average years of teacher experience was the most available data value and was included in datasets two 
and three. Other measures of teacher quality such as highest degree earned and status of certification were available in only a few cases and were not included. Data directly measuring building-level expenditures were also available in only two school districts. However, the number of teacher FTE per pupil was available in most cases and was included as a building-level, resource-related variable. Overall, 10 school-level variables were included for analysis.

In order to avoid inadvertent sub-sampling as the modeling proceeded, four discrete datasets were developed. Dataset one included the production rate of passing scores on AP Calculus posted by minority students plus the following time-averaged, school-level variables: proportions of minority and economically disadvantaged students, enrolled, total number of students enrolled, per pupil teacher FTE, achievement on state assessments of math and reading/language arts, and rates of production of passing scores on non-calculus AP exams by the total population. The second dataset included all of these variables plus timeaveraged years of teacher experience. As a result, dataset one included 407 data points from 133 schools enrollment approximately 221,000 students. Dataset two included 138 data points from 52 schools enrolling approximately 75,000 students. The contents of each dataset are described in Table 3. The modeling methodologies described above were applied to each dataset independently. Since a larger number of schools provide for a broader sample and more generalizable results, preference was given to models which were developed using datasets one and two. 


\section{Table 3}

\section{Definition of Datasets}

\begin{tabular}{llccc}
\hline Dataset \# & Variables Included & \# Schools & Ave Enrollment & Total Enrollment \\
Base & College Board Codes & 388 & 975 & 352,009 \\
0 & Minority Calc Production (MC) & 158 & 1591 & 251,503 \\
1 & MC, F1 through F7 & 133 & 1661 & 220,970 \\
2 & MC, F1 through F8 & 52 & 1434 & 75,467 \\
3 & MC, F1 through F9 & 40 & 1530 & 61,181 \\
4 & MC, F1 through F10 & 15 & 1808 & 27,126 \\
\hline
\end{tabular}




\section{CHAPTER 4}

\section{RESULTS}

Results of analysis are presented in this chapter. A general description of the base dataset is provided which showed an uneven distribution of minority Calculus production of passing scores on non-Calculus AP exams. An analysis of change over time with respect to the outcome variable was conducted using mixed models methodology. Results showed that, in general, no statistically significant change occurred over the time period investigated but that several time-averaged, school-level variables did have predictive power. In order to more directly investigate the effects of these school-aggregated factors on minority achievement in AP Calculus multi-variate regressions were performed. The analysis identified several school-level factors which held significant predictive power. The chapter ends with a discussion of key limitations inherent in this investigation.

\section{Descriptive Statistics}

Modeling primarily proceeded using time-averaged, school-level quantities. The computation of the primary and derived, time-averaged quantities is described in the previous chapter. Table 4 presents Pearson correlation coefficients computed for these quantities. The strongest statistically significant correlation reported here is between proportion of economically disadvantaged enrollment and proportion of minority enrollment $(+0.658)$. Schools represented 
in this sample which enroll a higher proportion of economically disadvantaged students also tend to enroll a higher proportion of Black and Hispanic students. Variables related to school-level achievement (average math and reading achievement and production rate of passing scores on non-Calculus AP exams) are strongly to moderately correlated to the outcome variable. Academic momentum is also strongly correlated to the outcome variable. However, this metric is constructed from changes over time in the three achievement variables just mentioned. Results from mixed models show that time is not an important predictor of the outcome variable, so this last correlation might only be a reflection of the correlations with the three original achievement variables. Variables related to demographics (ratio of economically disadvantaged students) and school resources (per pupil teacher FTE and per pupil expenditures) are also correlated with the outcome variable to a statistically significant level, as is total enrollment. These variables might therefore be expected to have predictive power with respect to the outcome variable and are therefore included in the fully-specified, time-averaged regressions presented in this chapter. 
Table 4

Correlations Between Time-Averaged Variables

\begin{tabular}{|c|c|c|c|c|c|c|c|c|c|c|c|}
\hline & 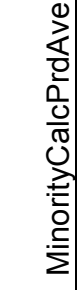 & 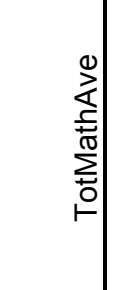 & 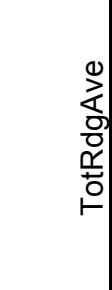 & 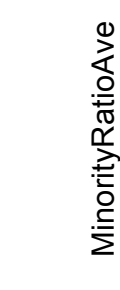 & 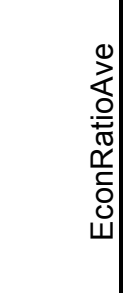 & $\begin{array}{l}\frac{0}{2} \\
\frac{1}{0} \\
0 \\
0 \\
\frac{0}{0} \\
0 \\
0\end{array}$ & 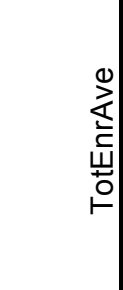 & 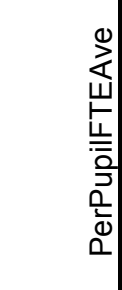 & $\begin{array}{l}\sum^{0} \\
\vdots \\
\sum_{0}^{0} \\
<\end{array}$ & 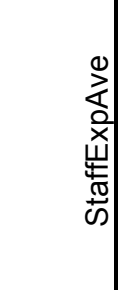 & 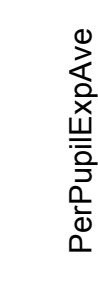 \\
\hline $\begin{array}{l}\text { Minority } \\
\text { Calc Prd } \\
\text { Ave }\end{array}$ & 1 & $.369^{* *}$ & $.236^{* *}$ & -.147 & $-.350^{* *}$ & $.547^{* *}$ & $-.192^{*}$ & $.197^{*}$ & $.461^{* *}$ & 101 & -.251 \\
\hline $\bar{N}$ & 133 & 133 & 133 & 133 & 133 & 133 & 133 & 133 & 114 & 52 & 21 \\
\hline $\begin{array}{l}\text { TotMath } \\
\text { Ave }\end{array}$ & & & $.569^{\star *}$ & -.076 & $\begin{array}{l}{ }^{* *} \\
-.471^{*}\end{array}$ & $.268^{* *}$ & .158 & $.172^{*}$ & .087 & .265 & -.235 \\
\hline$N$ & & 133 & 133 & 133 & 133 & 133 & 133 & 133 & 114 & 52 & 21 \\
\hline $\begin{array}{l}\text { TotRdg } \\
\text { Ave }\end{array}$ & & & $\begin{array}{r}1 \\
133\end{array}$ & \begin{tabular}{c}
\multicolumn{1}{c}{${ }^{* *}$} \\
-.235 \\
133
\end{tabular} & $-.341^{* *}$ & $.209^{*}$ & $\begin{array}{r}.099 \\
133\end{array}$ & $\begin{array}{r}.071 \\
132\end{array}$ & $\begin{array}{r}-.041 \\
114\end{array}$ & $.422^{* *}$ & $\begin{array}{r}.304 \\
21\end{array}$ \\
\hline$\frac{N}{\text { Minority }}$ & & & & 133 & $\begin{array}{r}133 \\
* *\end{array}$ & $\begin{array}{r}133 \\
* *\end{array}$ & 133 & 133 & 114 & \begin{tabular}{r|}
52 \\
$* *$
\end{tabular} & 011 \\
\hline $\begin{array}{c}\text { RatioAve } \\
N\end{array}$ & & & & $\begin{array}{r}1 \\
133\end{array}$ & & $\begin{array}{r}-.312 \\
133\end{array}$ & $\begin{array}{r}-.095 \\
133\end{array}$ & $\begin{array}{r}.164 \\
133\end{array}$ & $\begin{array}{r}-.059 \\
114\end{array}$ & $\begin{array}{r}-.466 \\
52\end{array}$ & $\begin{array}{r}-.041 \\
21\end{array}$ \\
\hline $\begin{array}{l}\text { EconRatio } \\
\text { Ave } \\
\quad N\end{array}$ & & & & & $\begin{array}{r}1 \\
133\end{array}$ & $\begin{array}{c}{ }^{* *} \\
-.453 \\
133\end{array}$ & $\begin{array}{r}-269^{* *} \\
133\end{array}$ & $\begin{array}{c}.225^{\star *} \\
133\end{array}$ & $\begin{array}{c}-266^{\star *} \\
114\end{array}$ & $\begin{array}{r}-.498^{* *} \\
52\end{array}$ & $\begin{array}{r}.137 \\
21\end{array}$ \\
\hline $\begin{array}{l}\text { TotAPPrd } \\
\text { Ave }\end{array}$ & & & & & & 1 & -.010 & -.171 & $.562^{* \star}$ & .239 & -.285 \\
\hline$N$ & & & & & & 133 & 133 & 133 & 114 & 52 & 21 \\
\hline $\begin{array}{l}\text { TotEnrAv } \\
\begin{array}{l}\text { e } \\
\quad N\end{array}\end{array}$ & & & & & & & $\begin{array}{r}1 \\
133\end{array}$ & $\begin{array}{c}* * \\
-.444 \\
133\end{array}$ & $\begin{array}{c}-.224^{*} \\
114\end{array}$ & $\begin{array}{r}.330^{*} \\
52\end{array}$ & $\begin{array}{r}-.428 \\
21\end{array}$ \\
\hline \begin{tabular}{|c} 
PerPupil \\
FTEAve \\
$N$
\end{tabular} & & & & & & & & $\begin{array}{r}1 \\
133\end{array}$ & $\begin{array}{r}-.102 \\
114\end{array}$ & $\begin{array}{c}{ }^{* *} \\
-.403 \\
52\end{array}$ & $.548^{*}$ \\
\hline $\begin{array}{l}\text { AcMom } \\
\text { AVe } \\
N\end{array}$ & & & & & & & & & $\begin{array}{r}1 \\
114\end{array}$ & $\begin{array}{r}.024 \\
40\end{array}$ & $\begin{array}{r}-.238 \\
15\end{array}$ \\
\hline \begin{tabular}{|c}
$\begin{array}{l}\text { StaffExp } \\
\text { Ave } \\
N\end{array}$ \\
\end{tabular} & & & & & & & & & & $\begin{array}{r}1 \\
52\end{array}$ & $\begin{array}{r}.313 \\
21\end{array}$ \\
\hline \begin{tabular}{|c|} 
PerPupil \\
ExpAve \\
$N$
\end{tabular} & & & & & & & & & & & 21 \\
\hline
\end{tabular}

**. Correlation is significant at the 0.01 level (2-tailed).

*. Correlation is significant at the 0.05 level (2-tailed). 
Measures of central tendency are shown for these quantities in Table 5. Three variables are worth taking special note of. Rates of Calculus production for minority students, rate of non-Calculus production for the total population, and academic momentum all have standard deviations that are larger than their respective means. Since none of these variables can take on negative values, they are therefore heavily skewed toward zero. This potentially problematic feature is shown in the histograms for Calculus production for the total population and for minority students. These histograms are shown in Figures 6 and 7 . In both cases, the data are heavily skewed toward 0 and are not normally distributed. While regression can proceed using data which are not normally distributed, these data can produce model residuals which are not normally distributed, a characteristic that is required in order that the $t$ tests on the computed coefficients be valid. Tests for normalcy of distribution of the residuals were therefore examined for each model. 


\section{Table 5}

\section{Descriptive Statistics of Time-Averaged Variables}

\begin{tabular}{|l|r|r|r|r|r|}
\hline & \multicolumn{1}{|c|}{$N$} & \multicolumn{1}{c|}{ Minimum } & Maximum & \multicolumn{1}{c|}{ Mean } & \multicolumn{1}{c|}{$S D$} \\
\hline MinorityCalcPrdAve & 133 & 0.4650 & 37.2652 & 4.9998 & 5.1709 \\
TotMathAve & 133 & 1.0000 & 100.0000 & 58.7950 & 25.8794 \\
TotRdgAve & 133 & 10.0000 & 99.9000 & 53.6258 & 23.1675 \\
MinorityRatioAve & 133 & 0.1005 & 0.9952 & 0.6894 & 0.2454 \\
EconRatioAve & 133 & 0.1255 & 0.9411 & 0.5822 & 0.2058 \\
TotAPPrdAve & 133 & 3.9118 & 1313.4975 & 175.1821 & 211.2473 \\
TotEnrAve & 133 & 111.7500 & 4169.6000 & 1661.4318 & 936.8115 \\
PerPupilFTEAve & 133 & 0.0290 & 0.1133 & 0.0560 & 0.0116 \\
AcMomAVe & 114 & 0.0400 & 362.7600 & 26.1859 & 44.4223 \\
StaffExpAve & 52 & 6.3600 & 19.0000 & 13.0439 & 2.8287 \\
PerPupilExpAve & 21 & 5282.1700 & 11184.0100 & 7565.0712 & 1402.1054 \\
Valid $N$ (listwise) & 15 & & & & \\
\hline
\end{tabular}

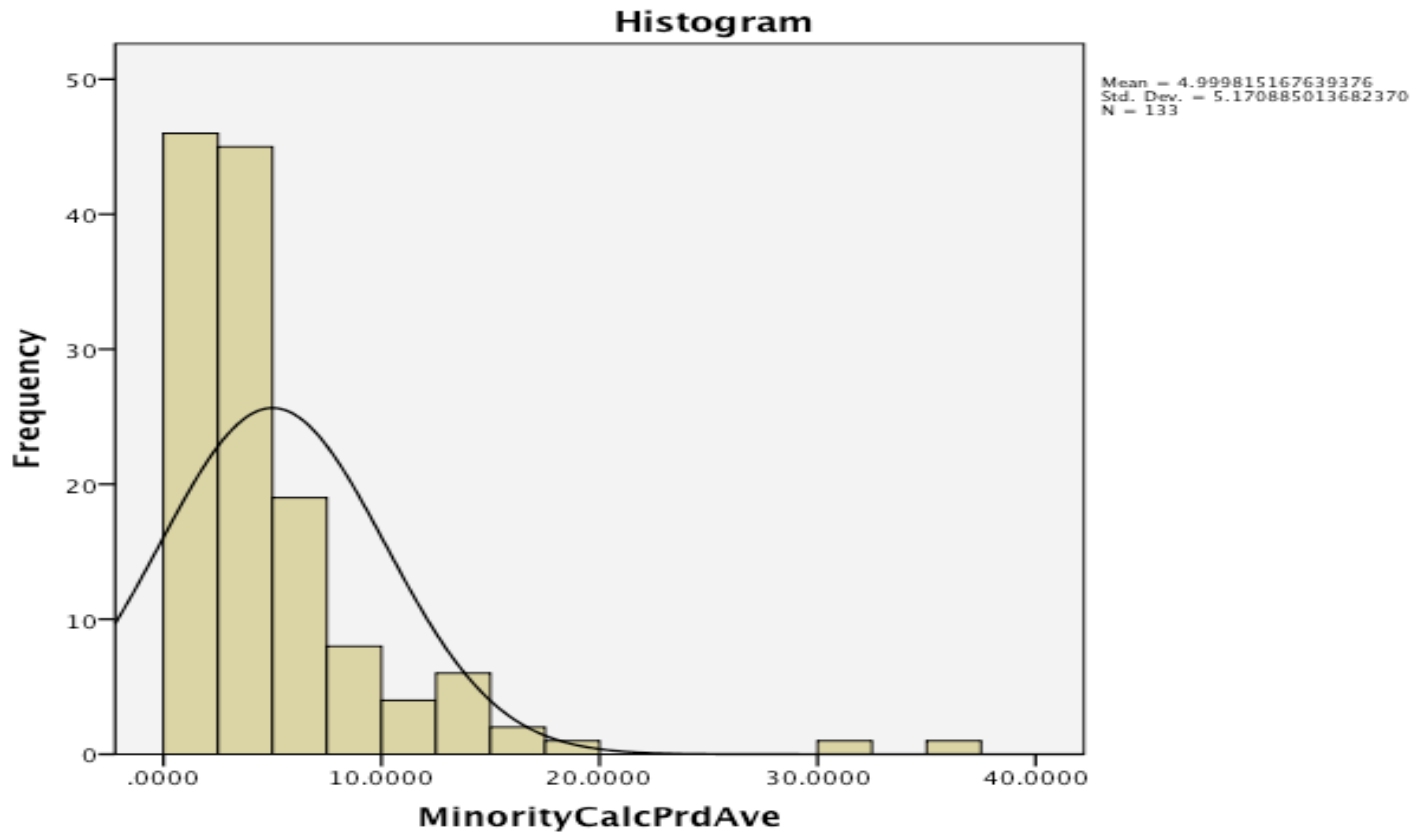

Histograms comparing distributions of AP-related time-averaged variables to normal distribution. Both distributions are strongly negatively skewed.

Figure 6. Comparison of minority AP production rates to normal distribution. 


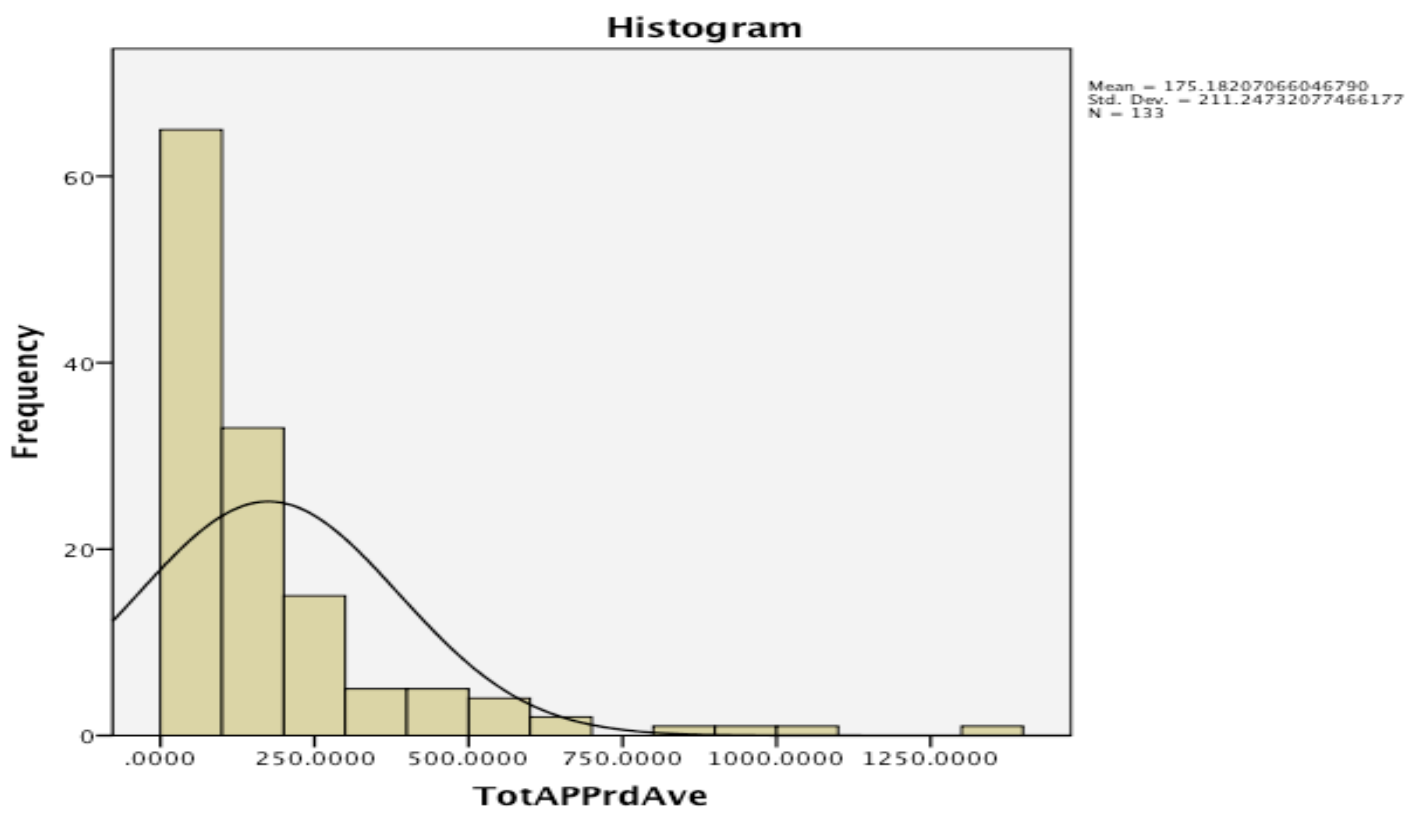

Histograms comparing distributions of AP-related time-averaged variables to normal distribution. Both distributions are strongly negatively skewed.

Figure 7. Comparison of total AP production rate to normal distribution.

\section{Unequal Distribution of Results}

One key characteristic of the database generated for this investigation is the uneven distribution of AP exam results across schools and school districts. Figure 8 shows percentages for all AP exams passed for the total population, AP Calculus exams passed for the total population, and AP calculus exams passed for minority students. These curves show that no AP exams of any sort are passed for a majority of the schools in this sample. It further shows that numbers of passing exams for AP Calculus are distributed more unevenly for minority students than for the total population. Figure 9 shows the cumulative number of AP Calculus exams passed for minority students. Those schools which account for $50 \%$ of the total passing scores for the respective groups are shaded. This 
figure shows that small fraction of the total schools (14 in total) account for half of the AP Calculus passing scores.

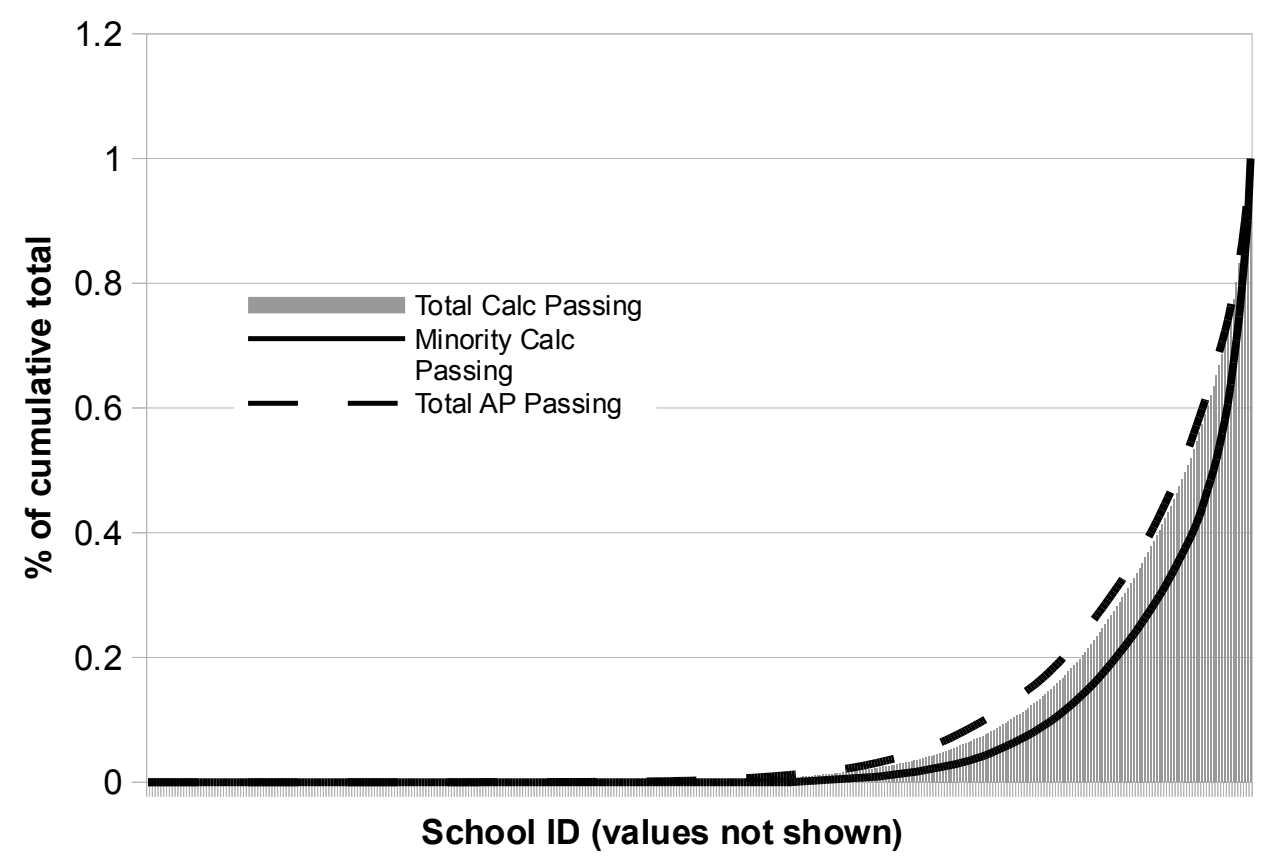

Cumulative histograms showing percentage of cumulative total exams passed for total population, AP Calculus exams passed for total population, and AP Calculus exams passed for minority population. Darker bars show that portion of the distribution which accounts for $50 \%$ of the total. Results show a highly uneven distribution across all schools.

Figure 8. Histogram of passing scores, total and minority populations. 


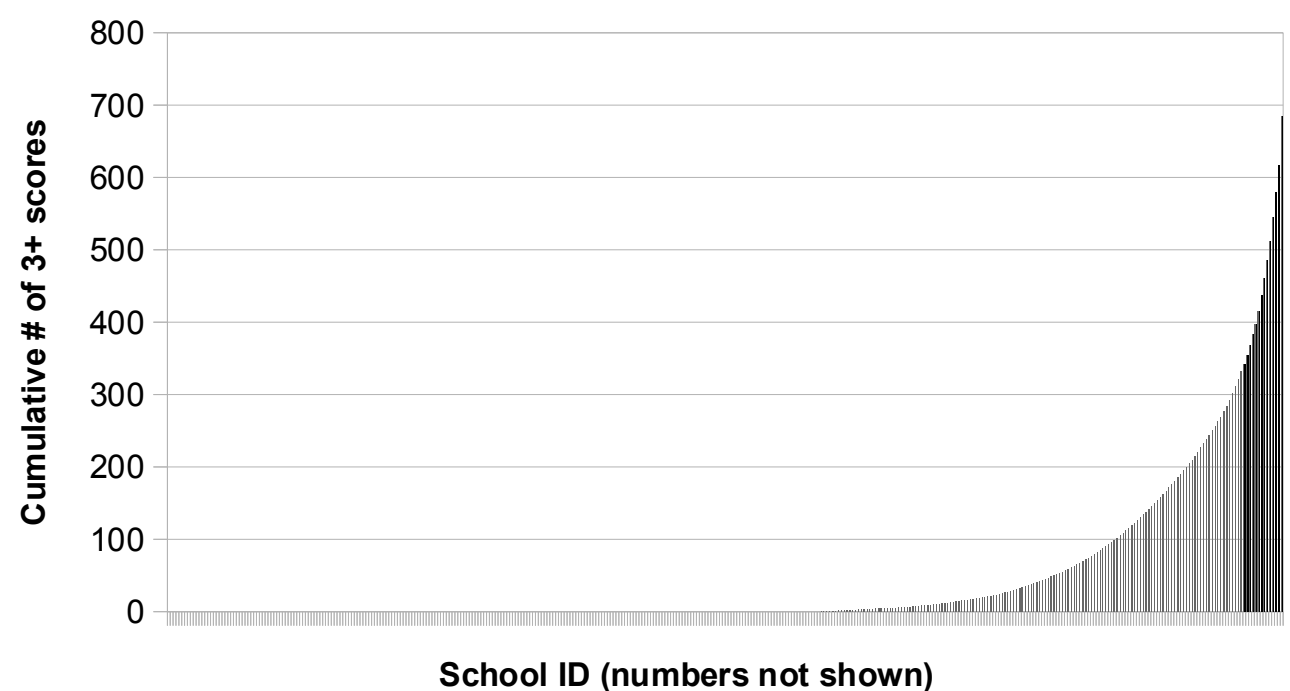

Cumulative histogram showing number of passing AP Calculus scores for minority students. Darker bars show that portion of the distribution which accounts for $50 \%$ of the total. Results show a highly uneven distribution across all schools.

Figure 9. Cumulative histogram of passing scores, minority students, AP Calculus

These 14 schools are examined in more detail in Table 6 and compared against the total sample. This table shows that minority students who attend the top 14 schools considered together pass more than 10 times the number of exams passed by minority students attending the rest of the schools in the sample. These schools are also larger, post much higher rates of proficiency on state assessments of mathematics and pass nearly five times as many nonCalculus AP exams. These schools are also somewhat less diverse and more affluent. All of these means are compared against the equivalent means for the 
total sample using Student's $t$ test and are all significantly different from the total sample at the $95 \%$ confidence level or better.

Table 6

Comparison Between Top Producers of Minority AP Calculus and the Total Population

\begin{tabular}{lllllll}
\hline & $\begin{array}{l}\text { Minority } \\
\text { Calc Tests } \\
\text { Passed }\end{array}$ & $\begin{array}{l}\text { Total } \\
\text { Enrollment }\end{array}$ & $\begin{array}{l}\text { Total Math } \\
\text { Achievemen } \\
\mathrm{t}\end{array}$ & $\begin{array}{l}\text { Total AP } \\
\text { Production }\end{array}$ & $\begin{array}{l}\text { Minority } \\
\text { Proportion } \\
\text { Enrolled }\end{array}$ & $\begin{array}{l}\text { Economic. } \\
\text { Disadv. } \\
\text { Proportion } \\
\text { Enrolled }\end{array}$ \\
$\begin{array}{l}\text { Top } 14 \\
\begin{array}{l}\text { Total } \\
\text { Population } \\
p \text {-value }\end{array}\end{array}$ & 1.76 & 907 & 35 & 94.2 & 0.56 & 0.66 \\
\hline
\end{tabular}

${ }^{* *}$ significant at 99\% level (2-tailed), ${ }^{*}$ significant at 95\% level (2-tailed), +significant at $90 \%$ level (2-tailed)

\section{Mixed Models to Analyze Change Over Time}

The effect of school-level, time-averaged factors on the rates of change over time on production of passing scores on AP Calculus exams by minority students was examined using a two-level mixed model methodology described in the previous section. Discrete sets of model runs were performed on datasets 1 through 4 as described in Table 7 in order to avoid inadvertent sub sampling as variables were added or removed. In each case, an unconditional, baseline individual growth model was developed and then compared against a fullyspecified growth model which included all the variables in the respective datasets. 


\section{Unconditional Individual Growth Model}

Results from the unconditional growth models are shown in Table 7.

\section{Table 7}

Comparison of Results for Unconditional Growth Models

\begin{tabular}{lcccc}
\hline & DS1 & DS2 & DS3 & DS4 \\
Intercept & $4.4902^{* *}$ & $3.1752^{* *}$ & $2.9246^{* *}$ & $2.3979^{* *}$ \\
Slope & $0.3080+$ & $0.3127+$ & $0.2747+$ & $0.1350+$ \\
Intercept- & $23.04711^{* *}$ & $4.9840^{* *}$ & $3.589^{*}$ & $3.2858+$ \\
Variance & 0.3840 & 0.0000 & 0.0195 & 0.1 \\
Slope-Variance & 2483.4630 & 740.2710 & 538.7040 & 203.0380 \\
AIC & & & & \\
\hline
\end{tabular}

** significant at 99\% level (2-tailed), *significant at 95\% level (2-tailed), +significant at 90\% level (2-tailed)

The intercept corresponds in each case to the average rate of production for passing minority calculus scores at time $t=0$. Time level zero is set at the 2007-2008 school year. Comparing intercepts therefore amounts to evaluating the values of the output variable during the 2007-2008 school year and positive slopes correspond to positive changes in the outcome variable between 20072008 and 2011-2012. Alternative choices for selecting time level zero are also possible. For example, time $t=0$ could be set at the 2011-2012 school year. Intercepts in that case would correspond to values of the outcome variable for the final year of analysis.

The values for the intercepts range from 4.49 to 2.39 and are significant at the $99 \%$ level. The between school variances for intercept are also significant, though only at the $90 \%$ level for dataset four. The estimate of slope corresponds 
to the average rate of change over time of production of minority calculus passing scores. All of these slopes are positive and significant at the $90 \%$ level. The between school variances, in contrast, are not significant. Taken together, these results suggest that significant between-school variation exists in initial conditions which might be explained by school-level factors. However, no significant between-school variation exists in slopes and the estimates of the slopes themselves are of dubious significance. In fact, at the $95 \%$ confidence level, these models fail to reject the null hypothesis with respect to slopes. Accepting the null hypothesis for slope (slope $=0$ ), exploring school-level factors that affect the average intercept amounts to examining school-level factors that affect time-averaged minority calculus production. This analysis is fact conducted using multi-variate regression later in this chapter.

\section{Fully-Specified Individual Growth Model}

In order to investigate what effect school-level factors might have on slopes and intercepts, a fully-specified model was developed independently for each dataset. Results, shown in Tables 8-10, show that estimates for time (slope) and intercepts failed to reject the null hypothesis. These results partially corroborate results from the unconditional models in that the rates of change over time of the outcome variable were not significant and presumed to be equal to 0 . Furthermore, between-school variances in slopes for the 4 models were also not significant. Finally, the interaction effects between time and the schoollevel predictors were not significant. Taken together, these results suggest that 
changes over time are not significant predictors of the production of passing scores on AP Calculus.

Table 8

Variances and Model Fit, Fully-Specified Growth Models

\begin{tabular}{lcccc}
\hline & DS1 & DS2 & DS3 & DS4 \\
Intercept & -4.3860 & 5.2641 & 4.5091 & -24.3409 \\
Slope & 1.1340 & 0.2776 & 1.0163 & 14.1886 \\
Intercept-Variance & $12.28^{* *}$ & 0.0000 & 0.0000 & 0.9890 \\
Slope-Variance & 0.17 & 0.0000 & 0.0186 & 0.0000 \\
AIC & 2448.51 & 733.7310 & 557.6200 & 226.1060 \\
\hline${ }^{* *}$ significant at 99\% level (2-tailed), *significant at 95\% level (2-tailed), +significant at 90\% level (2-tailed)
\end{tabular}

Table 9

Estimates of Coefficients, Fully-Specified Growth Models

\begin{tabular}{lcccc}
\hline & DS1 & DS2 & DS3 & DS4 \\
Intercept & NS & NS & NS & NS \\
Time & NS & NS & NS & NS \\
TotMathAve & NS & NS & NS & NS \\
TotRdgAve & NS & NS & NS & NS \\
MinorityRatioAve & NS & NS & NS & NS \\
EconRatioAve & $-8.11^{*}$ & $.004439^{* *}$ & NS & NS \\
TotAPPrdAve & $.00766^{* *}$ & NS & NS & NS \\
TotEnrAve & $-.001439^{*}$ & NS & NS & NS \\
PerPupilFTEAve & $191.699^{* *}$ & NS & NS & NS \\
StaffExpAve & & NS & NS & NS \\
AcMomAve & & & NS & NS \\
PerPupilExpAve & & & & NS \\
\hline
\end{tabular}

${ }^{* *}$ significant at 99\% level (2-tailed), ${ }^{*}$ significant at 95\% level (2-tailed), +significant at 90\% level (2-tailed) 
Table 10

Interaction Effects for Fully-Specified Growth Models

\begin{tabular}{lcccc}
\hline & DS1 & DS2 & DS3 & DS4 \\
TIme*TotMathAve & NS & NS & NS & NS \\
TIme*TotRdgAve & NS & NS & NS & NS \\
TIme*MinorityRatioAve & NS & NS & NS & NS \\
TIme*EconRatioAve & NS & NS & NS & NS \\
Time*TotAPPrdAve & $.001528^{* *}$ & NS & NS & NS \\
Time*TotEnrAve & NS & NS & NS & NS \\
Time*PerPupilFTEAve & NS & NS & NS & NS \\
Time*StaffExpAve & & NS & NS & NS \\
TIme*AcMomAve & & & NS & NS \\
TIme*PerPupilExpAve & & & & NS \\
\hline
\end{tabular}

** significant at 99\% level (2-tailed), ${ }^{*}$ significant at 95\% level (2-tailed), +significant at 90\% level (2-tailed)

In contrast, between-school variance in intercepts was significant for dataset one and was strongly impacted by the inclusion of the schoolaggregated, time-averaged predictors. Specifically, the variance in intercepts dropped by approximately $50 \%$, suggesting that school-level variables likely have predictive power with respect to the outcome variable. The results from dataset one indicate that ratio of economically disadvantaged students enrolled, the per pupil teacher FTE, the rate of production of passing scores on non-Calculus exams, and the total enrollment of the school are important predictors. 


\section{Partially-Specified Individual Growth Model}

A second set of tables shows the results of a separate set of models which were run in an effort to identify significant school-level predictors within each dataset (Tables 11 and 12). Each fully-specified model was optimized by eliminating each school-level predictor one at a time starting with the least significant variable (largest $p$-value) until only significant predictors remained. The choice of variables for the fully-specified model is described in the preceding chapter. The only predictor that remained in all four models was the rate of production of passing scores on non-Calculus AP exams. In all cases, this coefficient for this predictor was positive, indicating that as the rate of production of minority AP Calculus passing scores increased with the rate of production nonCalculus AP exams for the total population. In model for dataset one, the interaction effect between time and non-Calculus AP production was also significant.

Table 11

Results of Partially-Specified Growth Models

\begin{tabular}{lcccc}
\hline & DS1 & DS2 & DS3 & DS4 \\
Intercept & NS & NS & NS & NS \\
Time & NS & NS & NS & NS \\
TotMathAve & $.04962+$ & $0.4159+$ & & \\
TotRdgAve & & $-0.5480^{* *}$ & & \\
EconRatioAve & $-7.2136^{*}$ & & & $0.006707^{*}$ \\
TotAPPrdAve & $.00768^{* *}$ & $0.005423^{* *}$ & $0.005848^{* *}$ & \\
TotEnrAve & $-.001527^{*}$ & & & \\
PerPupilFTEAve & $181.2060^{* *}$ & & & \\
\hline
\end{tabular}

${ }^{* *}$ significant at $99 \%$ level (2-tailed), ${ }^{*}$ significant at 95\% level (2-tailed), +significant at $90 \%$ level (2-tailed) 
Table 12

Comparison of Interaction Effects for Partially-Specified Growth Models

\begin{tabular}{lcccc}
\hline & DS1 & DS2 & DS3 & DS4 \\
Time*TotMathAve & NS & NS & & \\
Time*TotRdgAve & & NS & & \\
Time*EconRatioAve & NS & & NS & NS \\
Time*TotAPPrdAve & $.001428^{*}$ & NS & & \\
Time*TotEnrAve & NS & & & \\
Time*PerPupilFTEAve & NS & & & \\
\hline
\end{tabular}

** significant at 99\% level (2-tailed), ${ }^{*}$ significant at 95\% level (2-tailed), +significant at 90\% level (2-tailed)

Table 13 shows a comparison between estimates for slopes, intercepts, model fit information, and variances for slopes and intercepts for each dataset. The Akaike's Information Criterion shows that model fit changed only slightly in all cases between the fully and partially-specified models. The intercept was significant for dataset one, but not for datasets two through four. As was true for the fully-specified models, slopes (rate of change over time for the outcome variable) was not significant and failed to reject the null hypothesis. The betweenschool variance for the intercept in dataset one stayed nearly the same between the fully and partially-specified models. However, when compared to the unconditional growth model, the variances in intercepts reduced by roughly $50 \%$. As was true for the fully-specified model, this result again suggests that schoolaggregated covariates have important predictive power with respect to the outcome variable. 
Table 13

Comparison of Model Outputs, Partially-Specified Growth Models

\begin{tabular}{lcccc}
\hline & DS1 & DS2 & DS3 & DS4 \\
Intercept & -2.6779 & $3.7063^{* *}$ & $1.7937^{* *}$ & $1.5170^{*}$ \\
Slope & 0.6276 & 0.1147 & 0.2478 & -0.1159 \\
Intercept- & $12.3606^{* *}$ & $7.6068^{* *}$ & 4.5679 & $1.1102^{* *}$ \\
Variance & & & & \\
Slope-Variance & 0.1796 & 0.0000 & 0.0000 & 0.0000 \\
AIC & 2446.6010 & 734.4980 & 523.7100 & 210.1550 \\
\hline
\end{tabular}

** significant at 99\% level (2-tailed), *significant at 95\% level (2-tailed), +significant at 90\% level (2-tailed)

The preceding analysis shows that time is not a salient predictor with respect to the production of passing scores on AP Calculus exams posted by minority students. To the limits of statistical significance, slopes (variation over time) failed to reject the null hypothesis. This does not indicate, however, that time is an unnecessary predictor in the level two equations. Except when values are actually constant, variations over time do occur within a longitudinal dataset. Those temporal variations may not differ significantly from zero across the entire sample and therefore may not produce significant estimates for slopes over time. Nonetheless, time must still be included as a control variable when estimating a longitudinal outcome variable. In the sections that follow, a time-averaged version of the outcome variable and time-averaged predictors are used. This renders the inclusion of time as a control variable moot. It is critical to emphasize, however, that the multi-variate regressions described in the next section are actually testing a different outcome variable. Conclusions about school level 
factors that impact time-averaged minority Calculus production are likely to apply to time-variant minority AP Calculus production as well, but this represents an extrapolation. It is safest to conclude that, as might be expected, no significant variations in the outcome variable occurred between the 2007-2008 and 20112012 school years for the schools included in the sample.

\section{Time-Averaged Regressions}

Results from the individual growth models developed above showed that time did not have a significant impact on the outcome variable for these schools over the time period under investigation. This result was perhaps expected given the relatively small, 5-year window of data being used. Changes in policy and teaching take time to manifest themselves. The absence of a time effect does not necessarily indicate that meaningful changes were not underway in the schools represented, only that, where they were in place, these changes had yet to manifest themselves to a statistically significant level as of 2012 .

In contrast, certain, time-averaged, school-level factors effectively reduced the variance in between-school variance in intercept. Given a zero slope over time, this shows that time-averaged, school-level quantities have predictive power with respect to time-averaged production of passing scores on AP Calculus posted by minority students. In order to directly examine the effects of time-averaged, school-aggregated predictors on time-averaged minority Calculus production, a series of multi-variate regressions were run on each previouslydefined dataset. The results are presented below. 


\section{Predictors of Minority AP Calculus Production}

As was the procedure for the growth modeling, regression modeling began with the fully-specified model for each dataset, the results of which are shown in Table 14. To optimize each model, variables were eliminated one at a time starting with the least significant variable (largest $p$-value) until all variables remaining were significant. Model quality was assessed via adjusted $R^{2}$ in order to account for the higher degree of fit which would be expected from a more complex model. The results of the partially-specified models are shown in Table 15. Note that only those variables which were significant are shown.

Table 14

Results of Fully-Specified Models, Time-Averaged Regressions

\begin{tabular}{lcccc}
\hline Variable/Parameter & DS1 & DS2 & DS3 & DS4 \\
$R^{2}$, Adjusted $R^{2}$ & $0.4490,0.4180$ & $0.8320,0.8010$ & $0.8040,0.7450$ & $0.8500,0.4760$ \\
TotMathAve & 0.1070 & $0.4850^{* *}$ & $0.5760^{*}$ & -0.1800 \\
TotRdgAve & 0.0190 & $-0.5040^{* *}$ & $-0.4960^{*}$ & 0.4090 \\
MinorityRatioAve & 0.1310 & $-0.2160+$ & $-0.34400^{*}$ & -0.5240 \\
EconRatioAve & $-0.2580^{*}$ & 0.0330 & 0.1650 & 0.4990 \\
TotAPPrdAve & $0.4740^{* *}$ & $0.5810^{* *}$ & $0.6860^{* *}$ & 0.2380 \\
TotEnrAve & $-0.1640^{*}$ & $-0.1970+$ & $-0.2110+$ & 0.5990 \\
PerPupilFTEAve & $0.2220^{* *}$ & -0.1000 & $-0.2310+$ & -0.2090 \\
StaffExpAve & & -0.0120 & 0.0260 & 0.3420 \\
AcMomAve & & & -0.1320 & 0.2310 \\
PerPupilExpAve & & & & 0.6250 \\
\hline
\end{tabular}

${ }^{* *}$ significant at $99 \%$ level (2-tailed), ${ }^{*}$ significant at 95\% level (2-tailed), +significant at $90 \%$ level (2-tailed) 
Table 15

Results of Regression for Partially-Specified Models, Time-Averaged Regressions

\begin{tabular}{lcccc}
\hline & DS1 & DS2 & DS3 & DS4 \\
$R^{2}$, Adjusted $R^{2}$ & $0.4190,0.4410$ & $0.8270,0.8080$ & $0.7950,0.7450$ & $0.8310,0.7370$ \\
TotMathAve & $0.1600+$ & $0.4500^{* *}$ & $0.5720^{* *}$ & \\
TotRdgAve & & $-0.5310^{* *}$ & $-0.4920^{* *}$ & $0.4090^{*}$ \\
MinorityRatioAve & & $-0.2210^{* *}$ & $-0.24400^{* *}$ & $0.4320^{*}$ \\
EconRatioAve & $-0.1530+$ & & & \\
TotAPPrdAve & $0.4690^{* *}$ & $0.5920^{* *}$ & $0.591^{* *}$ & \\
TotEnrAve & $-0.1580^{*}$ & $-0.157+$ & $-0.2160^{*}$ & $1.0780^{*}$ \\
PerPupilFTEAve & $0.214^{* *}$ & & $-0.234+$ & \\
StaffExpAve & & & & $0.3170^{* *}$ \\
AcMomAve & & & & $0.4370^{*}$ \\
PerPupilExpAve & & & & $0.8650^{*}$ \\
\hline ** significant at 99\% level (2-tailed), ${ }^{*}$ significant at 95\% level (2-tailed), +significant at $90 \%$ level \\
(2-tailed)
\end{tabular}

The governing equations for each of these fully-specified models are shown in Table 16. 
Table 16

Governing Equations for Fully-Specified Models, Datasets One Through Four

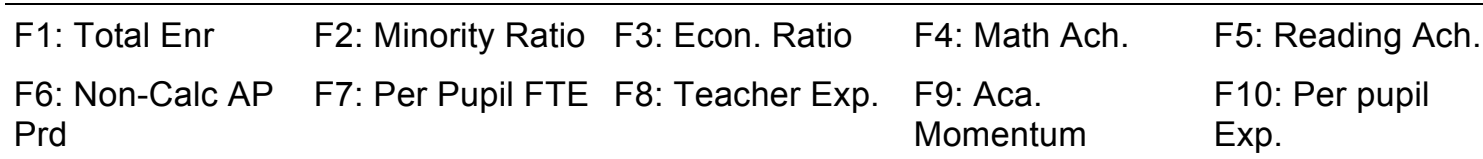

Dataset One (dependent variable minority AP Calculus production):

$$
Y_{j}=b 0+b 1^{*} F 1^{j}+b 2^{*} F 2_{j}+b 3^{*} F 3_{j}+b_{4}{ }^{*} F 4_{j}+b_{4}{ }^{*} F 4_{j}+b_{5}{ }^{*} F 5_{j}+b_{6}{ }^{*} F 6_{j}+b_{7}{ }^{*} F 7_{j}+r_{j}
$$

Dataset Two (dependent variable minority AP Calculus production):

$$
Y_{j}=b 0+b 1^{*} F 1^{j}+b{ }^{*} F 2_{j}+b 3^{*} F 3_{j}+b_{4}{ }^{*} F 4_{j}+b_{4}{ }^{*} F 4_{j}+b_{5}{ }^{*} F 5_{j}+b_{6}{ }^{*} F 6_{j}+b_{7}{ }^{*} F 7_{j}+b_{8}{ }^{*} F 8_{j}+r_{j}
$$

Dataset Three (dependent variable minority AP Calculus production):

$$
Y_{j}=b 0+b 1^{*} F 1^{j}+b 2^{*} F 2_{j}+b 3^{*} F 3_{j}+b_{4}{ }^{*} F 4_{j}+b_{4}{ }^{*} F 4_{j}+b_{5}{ }^{*} F 5_{j}+b_{6}{ }^{*} F 6_{j}+b_{7}{ }^{*} F 7_{j}+b_{8}{ }^{*} F 8_{j}+b_{9}{ }^{*} F 9_{j}+
$$

Dataset Four (dependent variable minority AP Calculus production):

$$
\begin{gathered}
Y_{j}=b 0+b 1^{*} F 1^{j}+b 2^{*} F 2_{j}+b 3^{*} F 3_{j}+b_{4}{ }^{*} F 4_{j}+b_{4}{ }^{*} F 4_{j}+b_{5}{ }^{*} F 5_{j}+b_{6}{ }^{*} F 6_{j}+b_{7}{ }^{*} F 7_{j}+b_{8}{ }^{*} F 8_{j}+b_{9}{ }^{*} F 9_{j+}+ \\
b_{10}{ }^{*} F 10_{j}+r_{j}
\end{gathered}
$$

Each successive dataset represents an overlapping subsample of the dataset above it. Additionally, of course, datasets do not contain the same variables. Since all models attempt to account for variations in the same outcome variable, consistencies between them would be expected. However, where inconsistencies appear, it cannot be assumed to be due to the addition of variables. Agreement and differences between the model results could emerge 
not as the result of the effects of additional variables but as the result of focusing the analysis on a smaller number of schools. This, in fact, would not be an unexpected result since the behavior of individuals cannot be reliably generalized to the behavior of the group.

Dataset four is the largest in terms of numbers of variables included and the smallest in terms of the number of data points available $(N=15)$. The fullyspecified model achieved a very high degree of fit $\left(R^{2}=0.850\right)$ and a strong adjusted coefficient of determination (adjusted $R^{2}=0.476$ ). However, none of the school-aggregated predictors were significant. The partially-specified model developed according to the procedure described earlier also achieved strong values for the coefficient of determination $\left(R^{2}=0.8310\right.$ and adjusted $R^{2}=$ 0.7370 ) and several significant predictors did emerge. These high levels of fit, however, are suspect given the very small data size. Furthermore, estimates of coefficients were inconsistent with results from datasets one through three in two important ways. First, the rate of the production of passing scores on nonCalculus AP exams did not remain as a significant predictor. In fact, it was the least significant predictor in the fully-specified model. In contrast, this predictor was the most significant predictor in the other models. Second, the three predictors which were not present in dataset one all remained as significant in the partially-specified model while in datasets 1 through 3 those predictors were eliminated. Additionally, the effect of time-averaged achievement on state assessments of mathematics was negatively related to the outcome variable in 
the fully-specified model, a result which is both inconsistent with datasets 1 through 3 and difficult to align with theory.

The governing equations for each of these partially-specified models are shown in Table 17.

\section{Table 17}

Governing Equations for Partially-Specified Models, Datasets One Through Four

\begin{tabular}{|c|c|c|c|c|}
\hline 1: Total & F2: Minority Ratio & F3: Econ. Ratio & $\mathrm{F} 4: \mathrm{Mc}$ & F5: Reading Ach. \\
\hline $\begin{array}{l}\text { 6: Non-Calc AP } \\
\text { rd }\end{array}$ & F7: Per Pupil FTE & F8: T & $\begin{array}{l}\text { F9: Aca. } \\
\text { Momentum }\end{array}$ & $\begin{array}{l}\text { F10: Per pupil } \\
\text { Exp. }\end{array}$ \\
\hline
\end{tabular}

Dataset One (dependent variable minority AP Calculus production):

$$
Y_{j}=b 0+b 1^{*} F 1^{j}+b 3{ }^{*} F 3_{j}+b_{4}{ }^{*} F 4_{j}+b_{4}{ }^{*} F 4_{j}+b_{6}{ }^{*} F 6_{j}+b_{7}{ }^{*} F 7_{j}+r_{j}
$$

Dataset Two (dependent variable minority AP Calculus production):

$$
\mathrm{Y}_{\mathrm{j}}=\mathrm{b} 0+\mathrm{b} 1^{*} \mathrm{~F} 1^{\mathrm{j}}+\mathrm{b} 2^{*} \mathrm{~F} 2_{\mathrm{j}}+\mathrm{b}_{4}{ }^{*} \mathrm{~F} 4_{\mathrm{j}}+\mathrm{b}_{4}{ }^{*} \mathrm{~F} 4_{\mathrm{j}}+\mathrm{b}_{5}{ }^{*} \mathrm{~F} 5_{\mathrm{j}}+\mathrm{b}_{6}{ }^{*} \mathrm{~F} 6_{\mathrm{j}}+\mathrm{r}_{\mathrm{j}}
$$

Dataset Three (dependent variable minority AP Calculus production):

$$
Y_{j}=b 0+b 1^{*} F 1^{j}+b 2{ }^{*} F 2_{j}+b_{4}{ }^{*} F 4_{j}+b_{4}{ }^{*} F 4_{j}+b_{5}{ }^{*} F 5_{j}+b_{6}{ }^{*} F 6_{j}+b_{7}{ }^{*} F 7_{j}+r_{j}
$$

Dataset Four (dependent variable minority AP Calculus production):

$$
Y_{j}=b 0+b 1^{*} F 1^{j}+b 2{ }^{*} F 2_{j}+b_{5}{ }^{*} F 5_{j}+b_{8}{ }^{*} F 8_{j}+b_{9}{ }^{*} F 9_{j+}+b_{10}{ }^{*} F 10_{j}+r_{j}
$$

The non-normal distribution of AP results across the schools described earlier necessitates an examination of residuals for each model. While regression analysis can proceed on non-normally distributed data, the $t$ tests used to assess 
the significance of the coefficients assume a normal distribution of residuals. Where residuals are not normally distributed, the computed $p$-values and the associated model specification process utilized here become suspect. A histogram and a $\mathrm{Q}-\mathrm{Q}$ plot of unstandardized residuals for dataset four is shown in Figures 10 and 11. The histogram in particular shows that unstandardized residuals are skewed toward negative values. These plots, in combination with the inconsistencies noted above and the very small sample size and the resultant problems with generalizability of results recommend modeling results from dataset four.

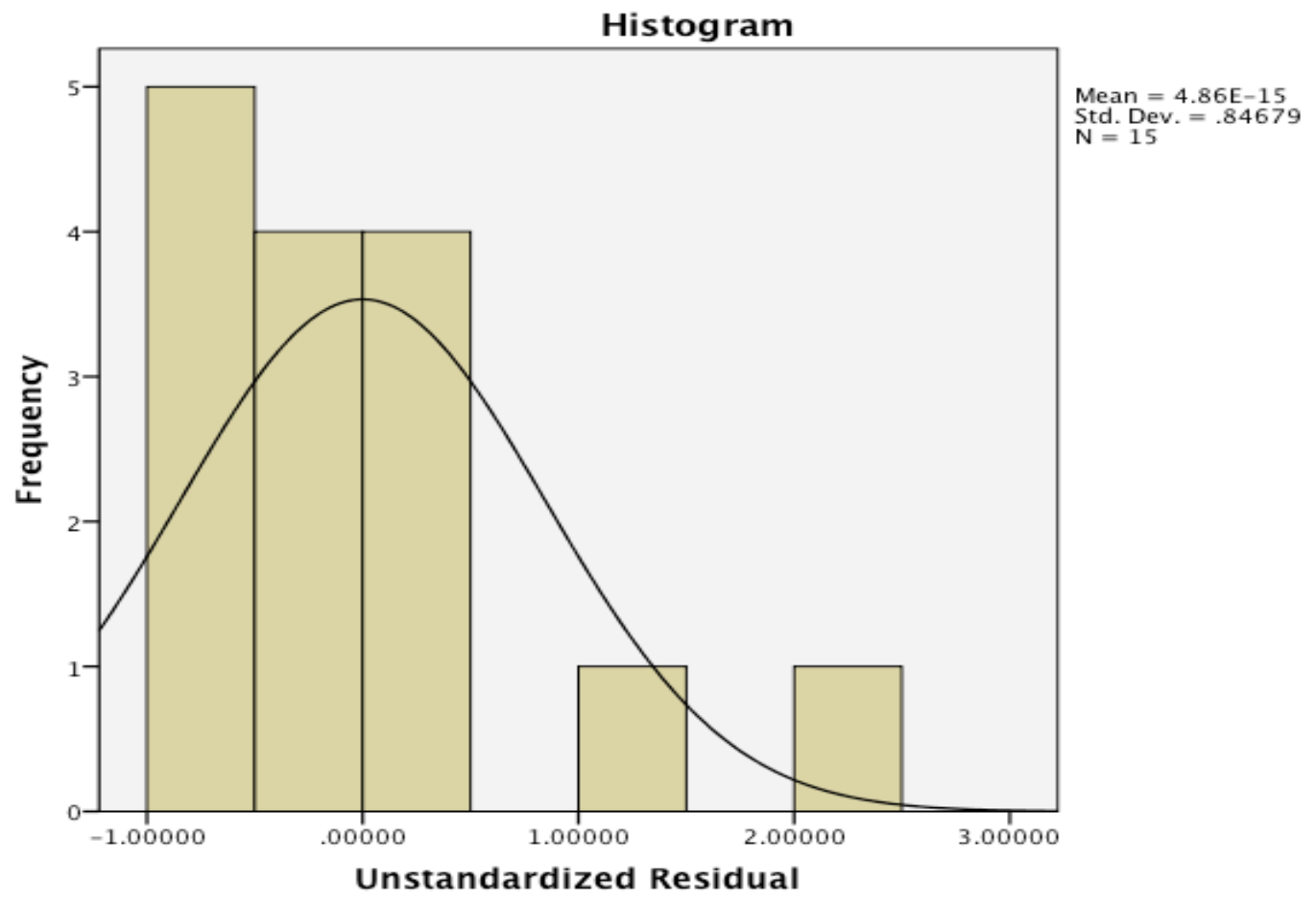

Figure 10. Histogram of unstandardized residuals, dataset four. 


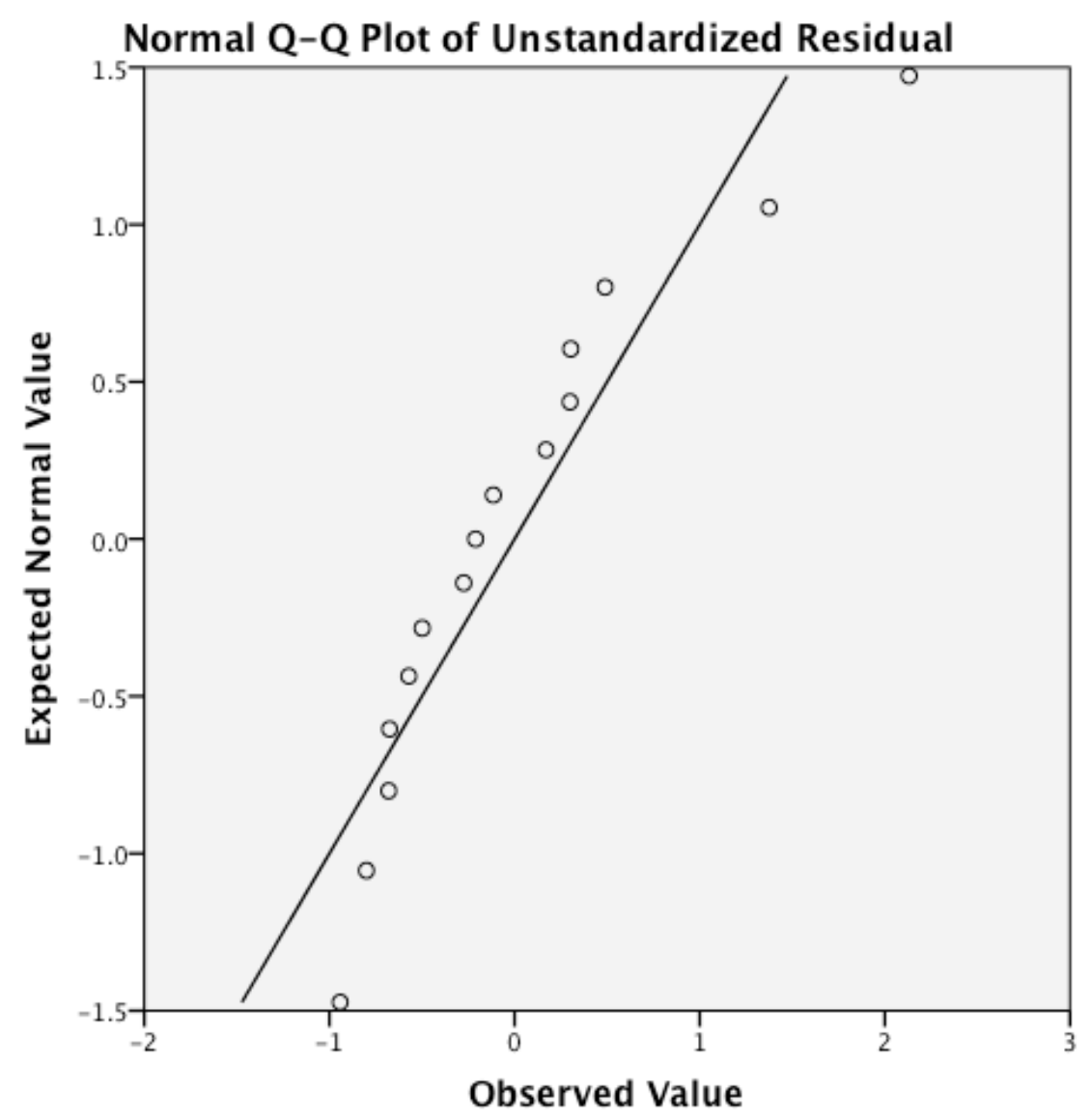

Figure 11. Q-Q plot of unstandardized residuals, dataset four.

Dataset three contained 40 schools and 9 potential school-aggregated predictors. The fully-specified model achieved high levels of fit with the data $\left(R^{2}=0.8040\right.$, adjusted $\left.R^{2}=0.7450\right)$ and several of the predicted coefficients were significant. Results from the fully and partially-specified models were also more consistent with results from datasets one and two. Specifically, production of passing scores on non-Calculus AP exams emerged as a significant predictor and the coefficients for years of teacher experience, academic momentum, and total building-level expenditures all failed to reject the null hypothesis. Total math 
achievement also remained in the partially-specified model as a positive predictor of minority Calculus production. Histograms and Q-Q plots depicted in Figures 12 and 13 show that unstandardized residuals for this model closely approach a normal distribution. However, the small sample size renders this model suspect of generalizability. Because of this, results from dataset three are included in the final discussion but are interpreted cautiously.

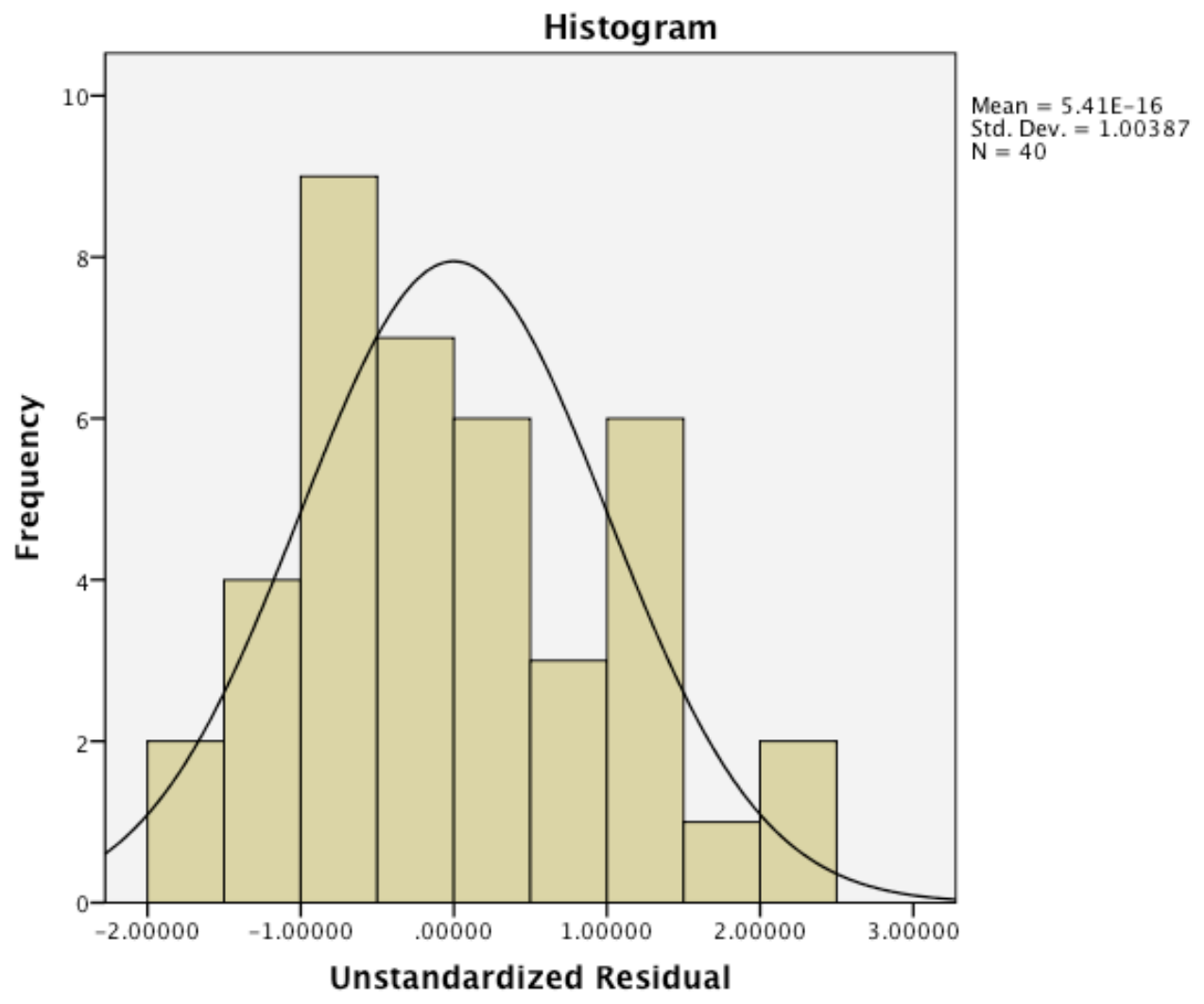

Figure 12. Histogram of unstandardized residuals, dataset three. 


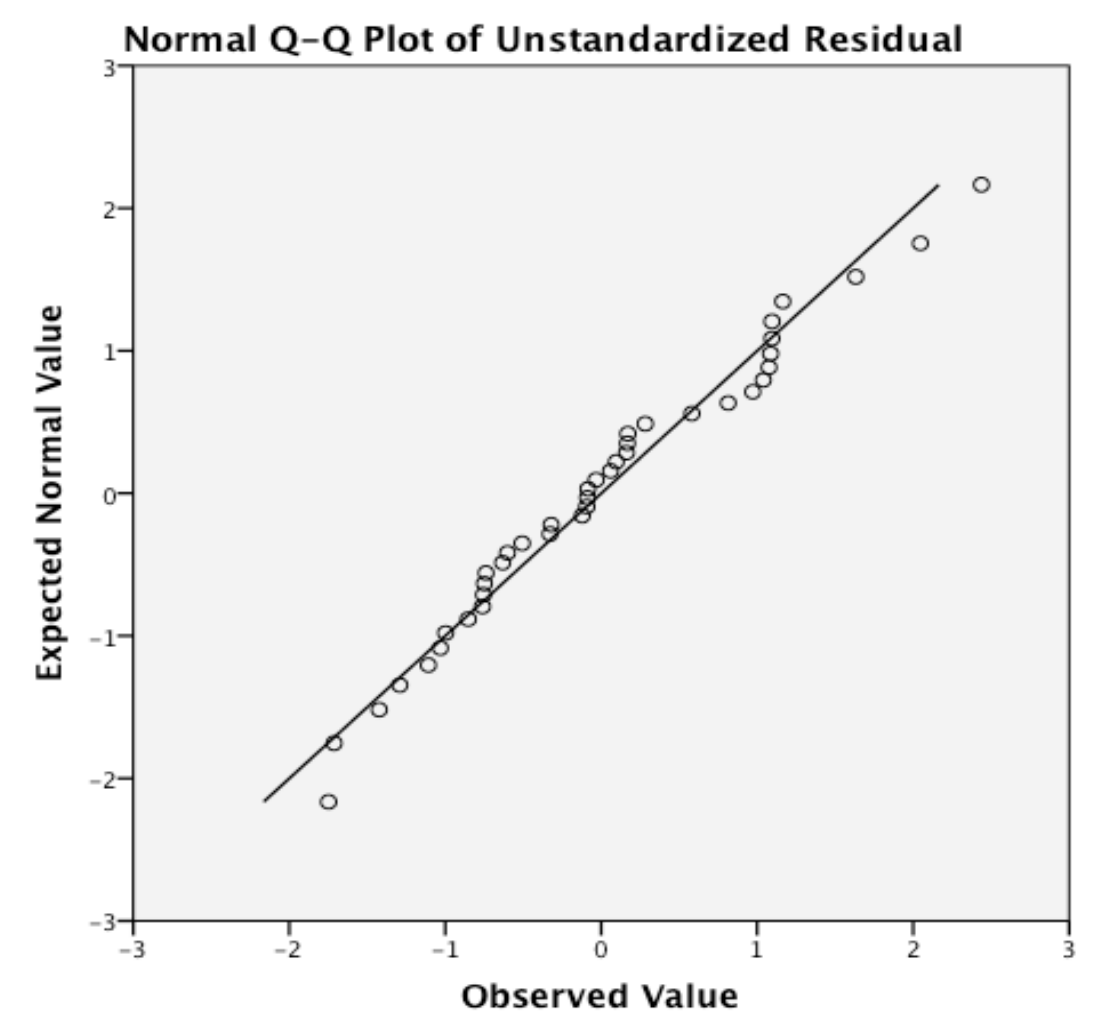

Figure 13. Q-Q plot of unstandardized residuals, dataset three.

Dataset two contains 52 schools in six schools districts and four major geographic regions. A total of 138 data points were used to compute the timeaveraged quantities used in this model. The generalizability of the models developed from dataset two are therefore better than for datasets three and four. In addition, results from the fully and partially-specified models are consistent with results from datasets one and three, most notably with regards to the sign and magnitude of the coefficients for total AP production and total enrollment. The coefficients for teacher experience, academic momentum and total buildinglevel expenditures also fail to reject the null hypothesis, results consistent with those obtained for datasets one and three. The histogram and Q-Q plot of 
unstandardized residuals for the partially-specified model for dataset two displayed in Figures 14 and 15 show a close to normal distribution, although important deviations are evident for four data points where the magnitude of the residuals were large.

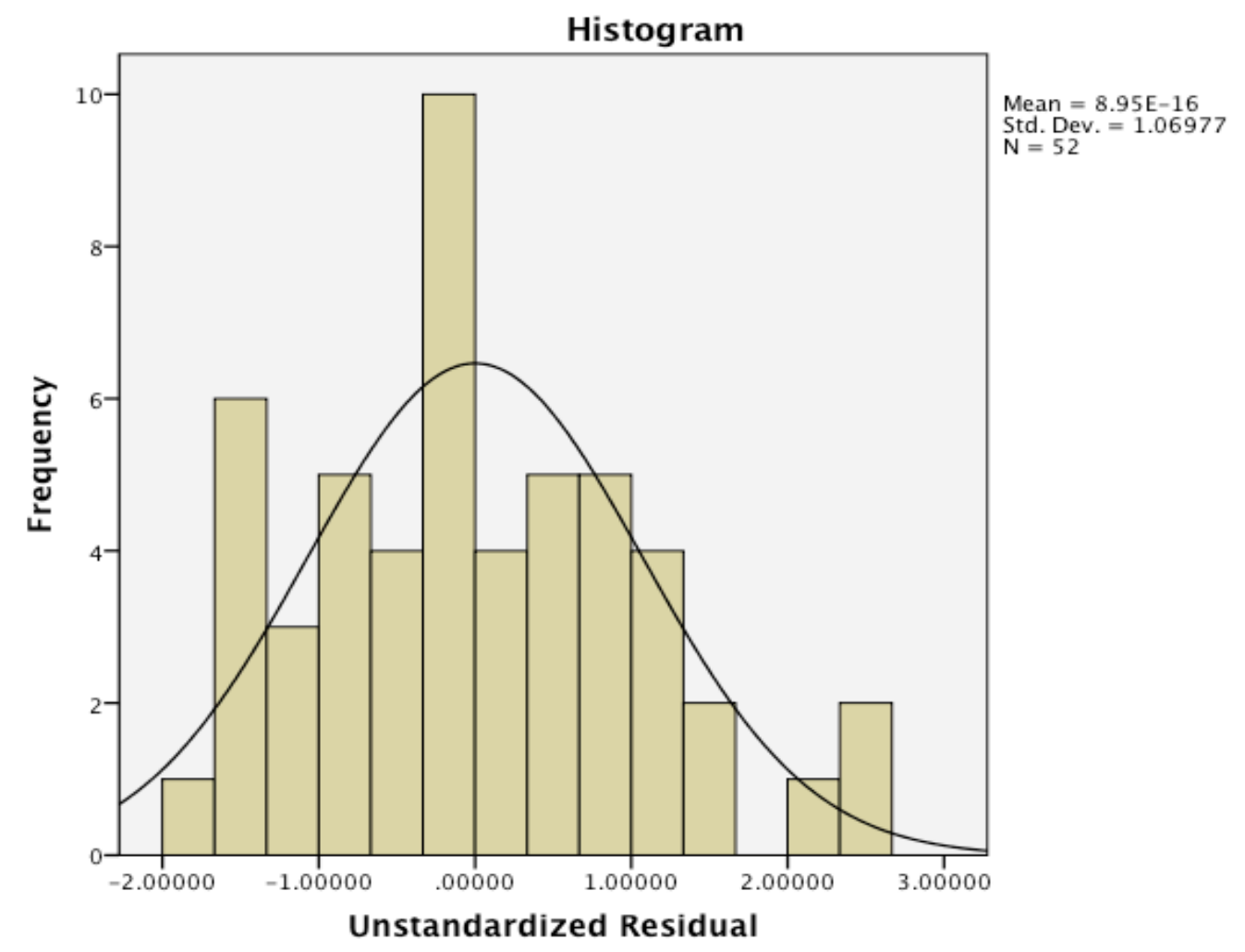

Figure 14. Histogram of unstandardized residuals, dataset two. 


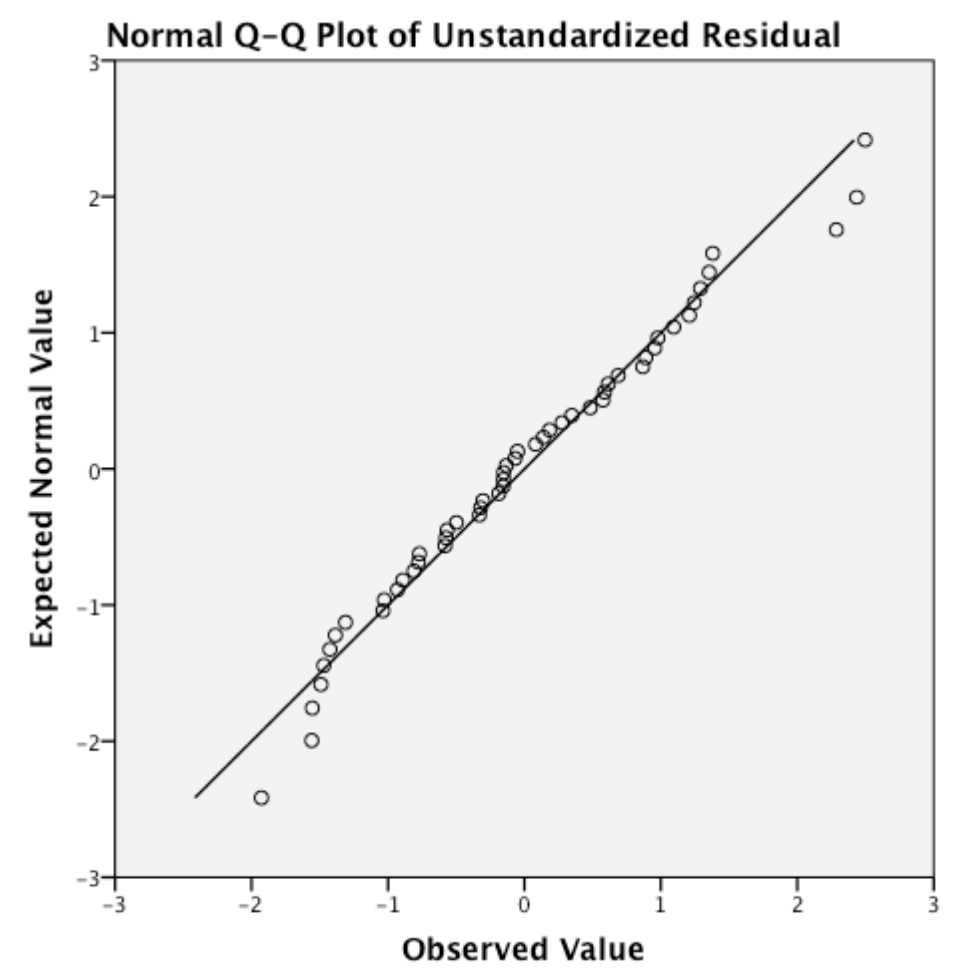

Figure 15. Q-Q plot unstandardized residuals, dataset two.

Dataset one contains 133 schools in nine school districts and seven geographic regions; 407 data points were available to compute the timeaveraged values for these regressions. The coefficients for teacher experience, academic momentum, and total building-level expenditures failed to reject the null hypothesis, and non-Calculus AP production was the strongest and most significant predictor. These results are consistent across datasets one, two, and three. The histogram and $Q-Q$ plots of the unstandardized residuals for dataset one depicted in Figures 16 and 17 show a similar pattern to those for dataset two. Specifically, residuals show a normal distribution with notable exceptions where the magnitudes of the residuals are large. In this case, 5 to 0 points show 
large deviations from a normal distribution, although the histogram shows fair agreement with the depicted normal curve.

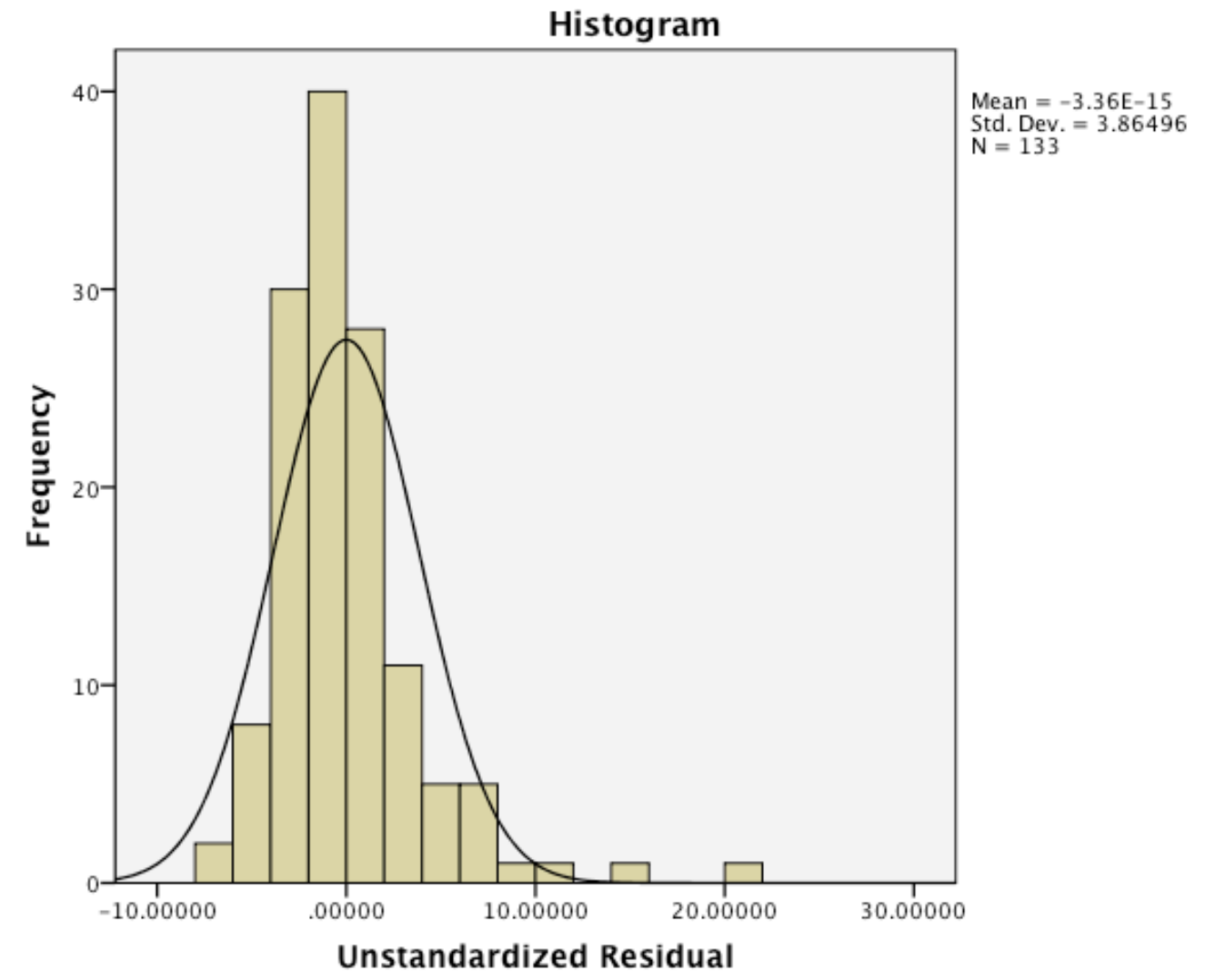

Figure 16. Histogram of unstandardized residuals, dataset one. 


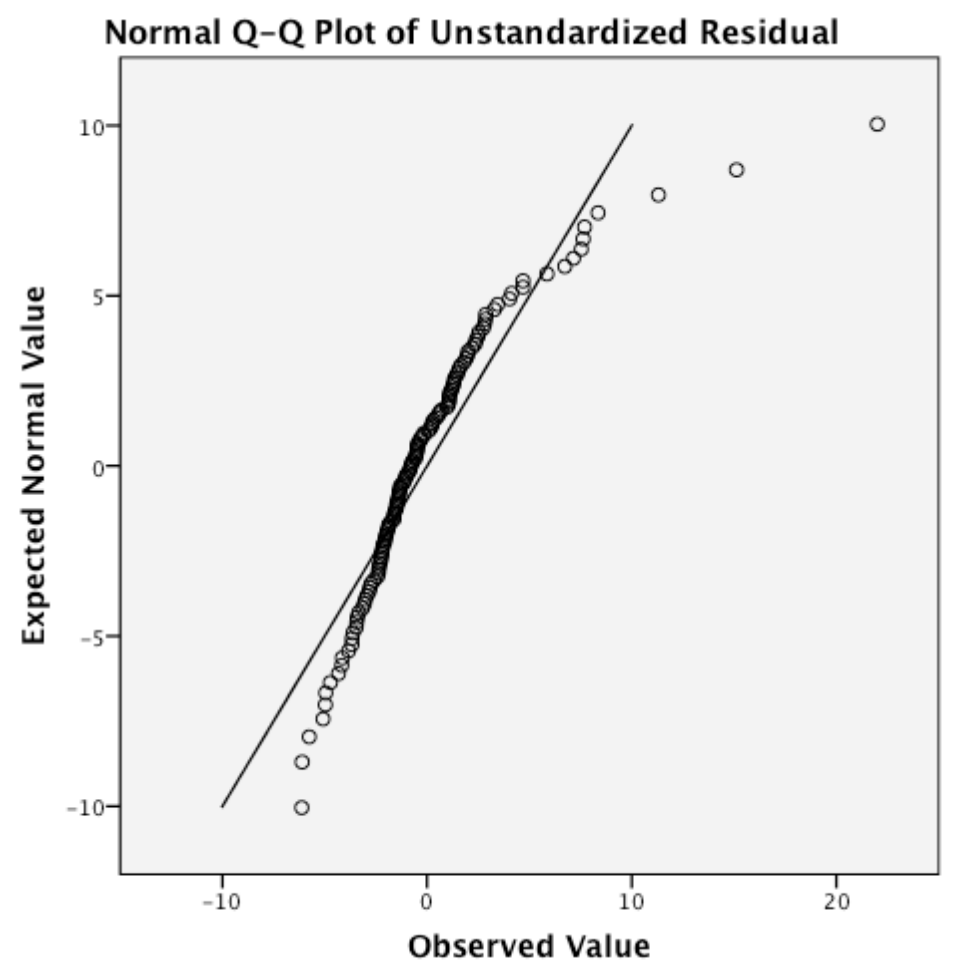

Figure 17. Q-Q plot of unstandardized residuals, dataset one

The partially-specified model for dataset one identifies non-Calculus AP production, total enrollment, total math achievement, per pupil teacher FTE, and proportion of economically disadvantaged students enrolled as significant predictors. Three inconsistencies between results for datasets one and two emerged. First, the partially-specified model for dataset one rejected total reading language arts achievement while the partially-specified model for dataset two retained total reading language arts achievement and estimated a much smaller value for the effect of total math achievement. In addition, the partially-specified model for dataset one estimated the coefficient for dataset one at only the $94 \%$ confidence level $(p$-value $=0.054)$. Second, per pupil teacher FTE was a 
significant predictor for dataset one but not for dataset two. Third, the proportion of economically disadvantaged students enrolled was a significant predictor for dataset one while the proportion of minority students enrolled was not. The opposite was true for dataset two.

The first and second of these effects operate together across all four datasets in an interesting way. The partially-specified model for dataset one includes per pupil FTE but not reading language arts achievement. The coefficient for math achievement is small and significant. The partially-specified model for dataset two does not include per pupil FTE but does include reading language arts and math achievement. The coefficient for math achievement is larger and more significant, while the coefficient for reading achievement is large, significant, and negative. The partially-specified model for dataset three contains all three variables (math, reading language arts, and per pupil FTE). The coefficient for math is large, significant, and positive. The coefficient for reading language arts is large, significant, and negative. The coefficient for per pupil FTE is moderate in magnitude, minimally significant ( $p$-value $=0.06$ ), and, contrary to the results for dataset one, negative in sign. The other major predictors stay relatively constant in magnitude and significance across these three datasets. These conditions suggest that some suppression is occurring in these regression caused by interactions between reading language arts achievement, math achievement, and per pupil teacher FTE. Although these three variables are not 
significantly correlated (see previous table of correlations), a more thorough analysis of these interactions might yield meaningful results.

The third of these effects is directly explainable through an exploration of the relationship between the proportion of economically disadvantaged students enrolled and the proportion of minority students enrolled. These two factors are strongly correlated for the schools represented in dataset one (Pearson coefficient $=0.658)$. In addition, a supplemental regression was run using data from the 359 schools for which relevant data were available. These results showed that the proportion of economically disadvantaged students enrolled alone accounted for $35 \%$ of the variation in proportion of minority students enrolled. These two time-averaged variables, therefore, carry much of the same information and can be expected to interfere with one another in the multi-variate regressions.

\section{Isolation of Variables in Dataset One}

The regression results from dataset one showed that achievement on state assessments of mathematics, per pupil teacher FTE, rates of production of passing scores on non-Calculus AP exams, proportion of economically disadvantaged students enrolled, and total enrollment have a significant impact on the outcome variable. As previously discussed, results for per pupil FTE are not consistent in significance and sign across datasets. In addition, while math achievement is consistent in significance and sign across these same datasets, results suggest that per pupil FTE, math achievement, and reading language arts 
achievement may be interacting in the regressions. In order to eliminate the possibility of these interactions and isolate which of the five significant variables listed above carries the largest predictive power, a series of supplemental regressions were carried out. The baseline regression (model 1A) eliminated per pupil FTE from the partially-specified model for dataset one but retained the rest of the predictors. That is, model $1 \mathrm{~A}$ utilizes dataset one and keeps total math achievement, proportion of economically disadvantaged students, total enrollment, and total non-Calculus AP production and independent variables. The remaining regressions (models $1 \mathrm{~B}$ through $1 \mathrm{E}$ ) eliminated one variable a time and left the other four variables intact. The comparisons between these supplemental models are shown in Table 18.

Table 18

Comparison of Results for Supplemental Analysis, Dataset One

\begin{tabular}{lccccc}
\hline & Model 1A & Model 1B & Model 1C & Model 1D & Model 1E \\
$R^{2}$ & 0.4110 & 0.3660 & 0.4040 & 0.2690 & 0.3530 \\
Standardized & 0.3930 & 0.3510 & 0.3900 & 0.2520 & 0.3880 \\
$R^{2}$ & & & $0.2800^{* *}$ & $0.2750^{* *}$ & $0.2290^{* *}$ \\
TotMathAve & $0.2430^{* *}$ & & $-0.3060^{* *}$ & -0.0280 \\
EconRatioAve & -0.1100 & $-0.2130^{*}$ & & & $0.4720^{* *}$ \\
TotAPPrdAve & $0.4290^{* *}$ & $0.4500^{* *}$ & $0.4690^{* *}$ & & \\
\hline TotEnrAve & $-0.2550^{* *}$ & $-0.2440^{* *}$ & $-0.2310^{* *}$ & $-0.3170^{* *}$ & \\
\hline
\end{tabular}

** significant at 99\% level (2-tailed), *significant at 95\% level (2-tailed), +significant at $90 \%$ level (2-tailed) 
These results show that the rate of production of passing scores on nonCalculus AP exams is the strongest predictor, accounting for approximately $14 \%$ of the total variation in the outcome variable. Math achievement accounted for approximately $4 \%$ in the variation while total enrollment accounted for approximately $1 \%$. When non-Calculus AP production was included, proportion of economically disadvantaged students enrolled had a generally small effect and it was not a significant predictor in models $1 \mathrm{~A}$ and $1 \mathrm{D}$.

The production of passing scores on non-Calculus AP exams for the total population was the largest and most significant predictor of minority production of passing scores on AP Calculus exams in all regressions conducted for datasets one through three. It is important to note that these variables are able to vary independently from one another. It would be possible for a school to have a high rate of production for non-Calculus AP exams and a low rate of production for minority AP Calculus exams. In fact this is the case for a number of schools in this sample. It is also important to note that the rate of minority Calculus production is normalized to the total minority population in a school, not the total population. This is to account for the possibility that schools with a high minority enrollment might naturally be expected to produce more minority passing AP Calculus scores. On a school level, then, the general rate of success nonCalculus AP exams for the total population is strongly predictive of rates of success for minority students on AP Calculus exams. It remains to be examined which school-aggregated factors predict success on non-Calculus AP exams for 
the total population. The second was a partially-specified, optimized model developed according to previously developed procedures. The results are shown in Table 19.

Table 19

Comparison of Fully- and Partially-Specified Models, Total Non-Calculus Production as Outcome Variables

\begin{tabular}{lcc}
\hline & Fully-specified & Partially-specified \\
$R^{2}$ & 0.2610 & 0.2590 \\
Standardized $R^{2}$ & 0.2260 & 0.2360 \\
TotMathAve & 0.1580 & $0.1560+$ \\
TotRdgAve & 0.0260 & \\
MinorityRatioAve & -0.0490 & \\
EconRatioAve & $-0.3510^{* *}$ & $-0.3950^{* *}$ \\
TotEnrAve & $-0.2330^{* *}$ & $-0.2360^{* *}$ \\
PerPupilFTEAve & $-0.2160^{*}$ & $-0.2140^{*}$ \\
\hline
\end{tabular}

${ }^{* *}$ significant at 99\% level (2-tailed), ${ }^{*}$ significant at 95\% level (2-tailed), +significant at 90\% level (2-tailed)

The governing equations for the fully-specified and partially-specified models are shown in table 20. 
Table 20

Governing Equations for Fully- and Partially-Specified Models, Non-Calculus and Dependent

\begin{tabular}{lllll}
\hline F1: Total Enr & F2: Minority Ratio & F3: Econ. Ratio & F4: Math Ach. & F5: Reading Ach. \\
F6: Non-Calc AP & F7: Per Pupil FTE & F8: Teacher Exp. & F9: Aca. & F10: Per pupil \\
Prd & & & Momentum & Exp.
\end{tabular}

Fully-specified (dependent variable non-Calculus AP production):

$$
Y_{j}=b 0+b 1^{*} F 1^{j}+b 3^{*} F 3_{j}+b_{4}{ }^{*} F 4_{j}+b_{5}{ }^{*} F 5_{j}+b_{7}{ }^{*} F 7_{j}+r_{j}
$$

Partially-specified (dependent variable non-Calculus AP production):

$$
Y_{j}=b 0+b 1^{*} F 1^{j}+b 3^{*} F 3_{j}+b_{4}{ }^{*} F 4_{j}+b_{4}{ }^{*} F 4_{j}+b_{7}{ }^{*} F 7_{j}+r_{j}
$$

The partially-specified model shows that the proportion of economically disadvantaged students enrolled and the total enrollment of the school are the most predictive variables included in this regression with respect to the outcome variable. When the proportion of economically disadvantaged students increased by one standard deviation, the rate of the minority AP Calculus production decreased by 0.395 standard deviations when all other variables are held constant. When the total enrollment increased by one standard deviation, the rate of the minority AP Calculus production decreased by 0.236 standard deviations after all other variables are held constant. Subsequent analysis showed that these two variables together account for $22 \%$ of the variation in the outcome variable. Subsequent analyses with the partially-specified model showed that important interactions occurred between per pupil teacher FTE and total math achievement, corroborating earlier results. As a final check on model 
validity, a Q-Q plot and histogram of unstandardized residuals for the partiallyspecified model are shown in Figures 18 and 19. Results generally show a normal distribution of residuals, although large variations are apparent for four data points with large-magnitude residuals. This behavior was also noted in the residuals for dataset one, the impact of which is discussed more fully in the final chapter. Stated briefly, since the model specification process relies on levels of significance of the coefficients, and since these $p$-values rely on the normal distribution of residuals, non-normal distributions like the ones shown here have the potential to alter the formulations of the final models. To correct this, a subsequent analysis utilizing a transformation of the AP-related variables is required. 


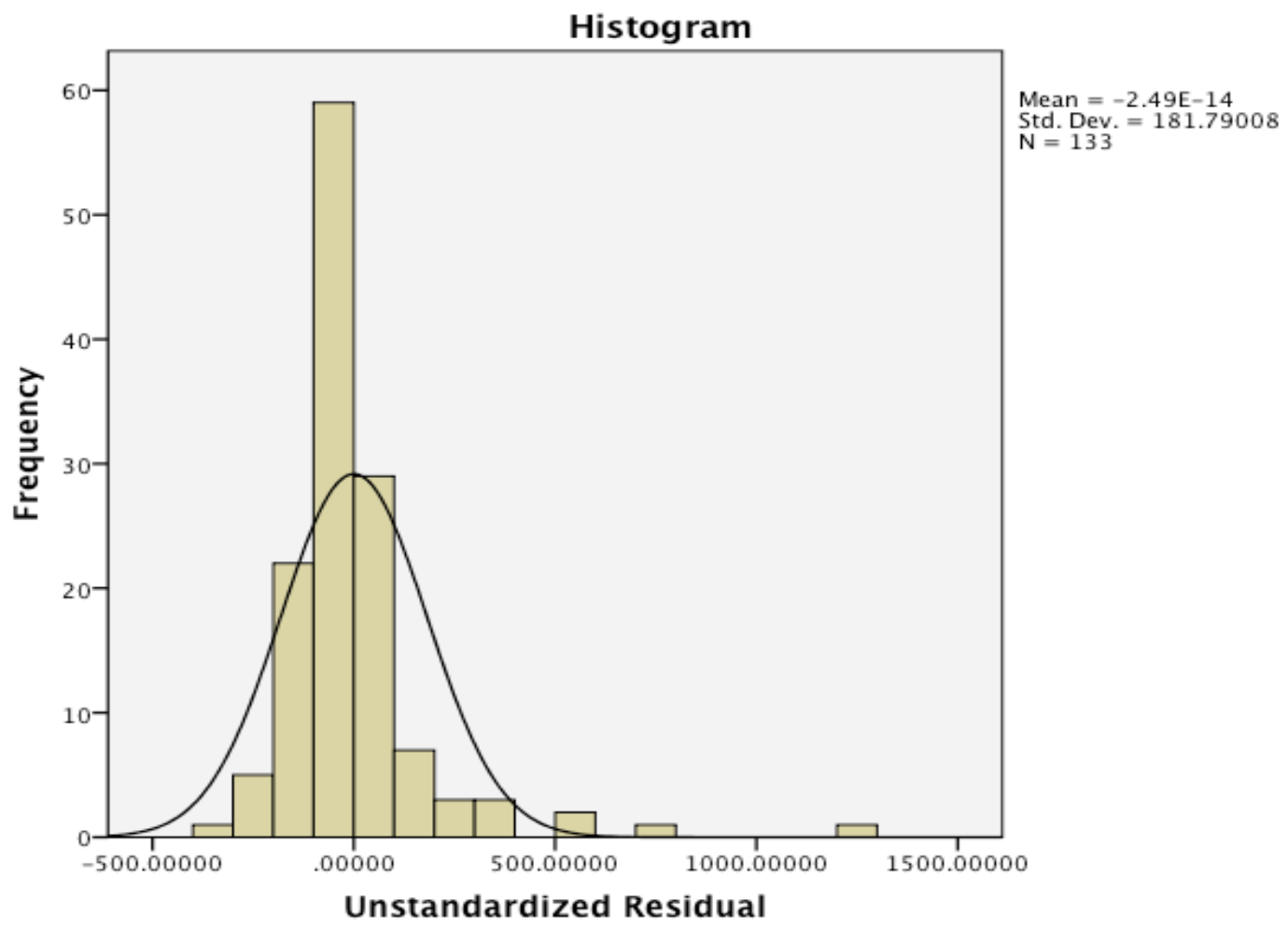

Histogram of unstandardized residuals for the partially-specified model, non-Calculus AP production as the Dependent Variable. Results show important deviation from normal distribution.

Figure 18. Histogram of unstandardized residuals for partially-specified model, non-Calculus AP production. 


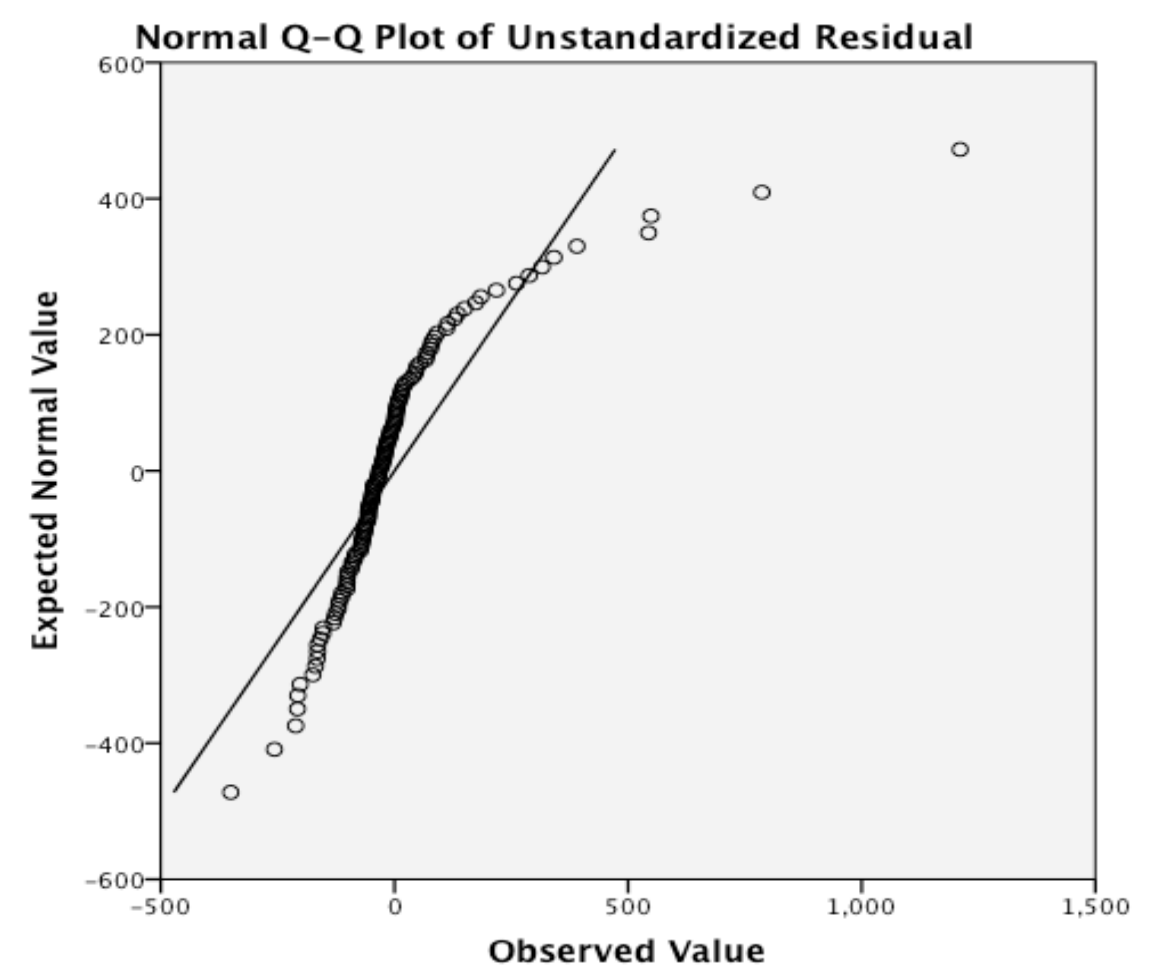

Q-Q plot of unstandardized residuals for the partially-specified model, non-Calculus AP production as the Dependent Variable. Results show important deviation from normal distribution.

Figure 19. Q-Q plot of unstandardized residuals for partially-specified model, non-Calculus AP production.

\section{Total AP Participation}

Ensuring that students pass AP exams is much more difficult from a policy and procedure perspective that ensuring that students take AP exams. If taking more AP exams automatically resulted in passing more AP exams, then school planners would only have to ensure that every student in the school takes multiple AP exams. The relationship between the rate of taking AP exams and the rate of passing AP exams is therefore of some interest. These two variables are, of course, not independent of one another. It is not possible to pass an exam 
that is never administered. On the other hand, a 1:1 relationship would also not be expected. Taking more exams does not automatically result in passing more exams.

A supplemental analysis using production of passing scores on nonCalculus AP exams as the dependent variable and the total number of all AP exams taken normalized to the total population as the independent variable shows that the number of exams taken accounts for $87 \%$ of the variation in total non-Calculus exams passed. The value of unstandardized coefficient $(b=0.634)$ represents the global, time-averaged "pass rate" for these schools, indicating that students, on average, pass $63 \%$ of the exams they take. This result may simply be a reflection of the selective nature of AP course offerings and enrollments. It is interesting to note, however, that one final set of supplemental regressions showed that the proportion of economically disadvantaged students enrolled and the total enrollment of the school were both significantly and negatively related to the rate of taking AP exams.

\section{Summary of Results}

A set of descriptive statistics, a series of multi-level individual growth models, and a set of regressions on time-averaged quantities are presented above. The goal of these analyses was to identify school-aggregated factors that impact the rate of production of passing scores on AP Calculus exams posted by minority (Black and Hispanic) students. Correlation analysis showed that the proportion of minority students enrolled was highly and significantly correlated 
with the proportion of economically disadvantaged students enrolled. Descriptive statistics also showed that AP achievement in general and AP Calculus achievement specifically is not evenly distributed. A relatively small number of schools account for the bulk of all AP achievement. As one example, just 14 of the 388 schools analyzed accounted for $50 \%$ of the passing AP Calculus scores posted by minority in a time-averaged sense.

One key argument advanced was that important and systematic temporal variation exists in the rate of production of minority AP Calculus passing scores and that school-level factors impact these rates of change. Individual growth models effectively disproved this contention and showed that, over the time period being analyzed (the 2007-2008 through 2011-2012 school years), no significant variation over time was evident. However, these same analyses showed that time-averaged, school-level variables effectively reduced betweenschool variance in initial conditions. Allowing that rate of change over time in the outcome variable is zero, this amounts to showing that time-averaged, schoollevel variables have predictive power with respect to time-averaged, school-level minority AP Calculus production.

In order to investigate this more fully, a series of multi-variate regressions were conducted using time-averaged quantities. Results showed that total enrollment and the rate of production of passing scores on non-Calculus AP exams were consistently and significantly related to minority AP Calculus production. In all cases, total enrollment was negatively related and non-Calculus 
AP production was positively related to the outcome variable. Results also suggested that per pupil teacher FTE, total mathematics achievement, and total reading language arts achievement interacted, recommending a potential subsequent area for investigation within these datasets.

Analyses using dataset one showed that the proportion of economically disadvantaged students enrolled was a stronger a more significant predictor of minority Calculus and total non-Calculus AP production than was the proportion of minority students enrolled. The proportion of economically disadvantaged students enrolled was also significantly and negatively related to the rate of taking AP exams as was total enrollment of the school. Taken together, the results of these regressions suggest that the SES of the school and its total enrollment are the strongest predictors of the rates of taking AP exams, passing non-Calculus AP exams, and the rates of passing Calculus exams by minority students. The implications of these findings and the key limitations of these analyses are discussed in the next chapter. 


\section{CHAPTER 5}

\section{DISCUSSION}

\section{Summary of Key Findings}

A number of assertions made in the earlier sections of this document were not borne out by the analysis. The hypotheses advanced were all related to changes over time of the production of the passing scores on AP Calculus posted by minority students. Implicit in these hypotheses was the assumption that systematic change over time did occur in the outcome variable and that these rates of change were malleable under the influence of school-level variables. This assumption was not evident in the data. In all cases except the unconditional growth models, the estimates for slope (change over time) and between-school variance in slope failed to reject the null hypothesis, indicating that, over the time period investigated, no temporal changes in the outcome variable were apparent. While counter to expectations, this finding carries relevant information. For schools reporting non-zero minority AP Calculus success, changes in policy or practice had not been implemented or, where implemented, had yet to have appreciable effect over the time period investigated. Minority success in AP Calculus appears to be a relatively stable phenomenon for the schools included between 2007-2008 and 2011-2012 school years. Additional data and analyses are required in order to determine the 
potential relationship between this observation and the implementation of policy and practice.

Hypothesis two posited that variables relating to school resources would be positively related to the growth in achievement and participation of Minority students in AP Calculus. While, as noted above, no effects over time were noted, the impact of resource-related school-aggregated variables was investigated as part of the time-averaged regressions. Financial expenditure data were available in only two school districts. The requirement that outcome variable be non-zero also limited the size of the sample. The union of these two sets left only 15 schools for analysis. The modeling which resulted from this dataset (dataset four) was rejected for reasons described in the analysis section. However, per pupil FTE constitutes a reasonable proxy as a resource-related variable, and this information was more widely available. Dataset one included this variable and was the largest of the datasets. Modeling using this dataset found per-pupil teacher FTE to be a significant and positive predictor of minority AP Calculus production in the fully-specified model. Specifically, when the number of teacher FTE increased by one standard deviation, the rate of production of passing scores in minority AP Calculus increased by 0.222 standard deviations. This finding partially corroborates hypothesis two and indicates that where resources are directed toward increasing the ratio of teacher FTE to total enrollment, the rate of minority AP Calculus production increases when controlling for the other variables included (see previous section). 
The partially-specified model found similar results for per pupil teacher FTE $\left(b=0.214^{\star *}\right)$ when controlling for math achievement for the total population, total enrollment, the proportion of economically disadvantaged students, and the rate of production of passing scores on non-Calculus AP exams for the total population. These results agree with the previous investigations cited earlier showing that disparities in school resources resulted in lower achievement on standardized measures of achievement (Condron \& Roscigno, 2003; Payne \& Biddle, 1999) and lower salaries and poorer working conditions for staff (Roza et al., 2004). The current investigation adds to these results, showing that per pupil teacher FTE has a positive and significant impact on minority AP Calculus production.

It is important to note that significant interaction effects appeared to be in play between per pupil teacher FTE, total math achievement, and total reading language arts achievement. These interaction effects are, in fact, predicted by the investigators just cited and would be expected to be in place. Their existence, however, does complicate the analysis. Results from dataset one found positive and significant effect between per pupil teacher FTE and minority AP Calculus production while the results from dataset three found a significant and negative relationship between these variables. This flip in sign suggests that the inclusion or exclusion of math and reading language arts achievement strongly impacts the magnitude and direction of the effect of per pupil FTE and that the variables may 
interact in a systematic way. Additional analysis is required in order to more thoroughly explore these interactions.

Modeling results for datasets one through three found that the proportion of economically disadvantaged students and the proportion of minority students enrolled were significantly and negatively related to minority AP Calculus production for the partially-specified models. These results corroborate the findings of earlier researchers who showed that family SES (Reardon et al., 2012) and school-aggregated SES (Engberg \& Wolniak, 2010; Ma \& Klinger, 2000) negatively impacted mathematics achievement, academic achievement, and college enrollment. The school's ethnic composition has been shown to be an important predictor of student achievement (Coleman et al, 1966; Rumberger \& Palardy, 2005). Students' SES and ethnicity can also become bases for stereotype threat (Croizet \& Claire, 1998; Steele \& Aronson, 1995) and have the potential to activate "ego defensive" mechanisms like oppositional, all of which are predicted to suppress academic achievement culture (Allport, 1954; Fordham \& Ogbu, 1986)

The results of this investigation with respect to school-aggregated SES and ethnic composition, while statistically significant and supported by existing theory, must also be interpreted with caution. The coefficient for the proportion of economically disadvantaged students enrolled flipped signs and become insignificant between datasets one and two while the coefficient for proportion of minority students enrolled showed the reverse result. The proportion of minority 
enrollment and the proportion of economically disadvantaged students enrolled were strongly correlated, so these interaction effects were expected but also complicated the analysis. The results support only a provisional conclusion with respect to school-aggregated SES and ethnicity. Specifically, as the proportion of minority and economically disadvantaged students enrolled increased, the rate of production of minority AP Calculus students decreased.

Three results did emerge strongly from this analysis. First, the distribution of AP results across the schools investigated is extremely uneven. A small fraction of schools in this sample account for the great majority of AP participation and AP success for both Calculus and non-Calculus exams while most schools reported very small numbers of AP exams administered and passed. Just 26 schools account for $50 \%$ of all passing scores on all AP exams and 14 schools account of $50 \%$ of all minority AP Calculus passing scores in a time-averaged sense. A comparative analysis shows that this latter group of 14 top performers is, on average, more affluent, less diverse, and larger than schools in the general population. The very small size of this sub population further suggests that specific policies, procedures, and philosophies might be in place in those schools which constitute powerful predictors of minority AP Calculus production. An effect of this sort would potentially be detected through an analysis of comparator schools.

School size also emerged as a significant and negative predictor of minority Calculus production. This finding is supported by theory on a number of 
levels. First, a number of researchers have shown that minority and low-SES students are disproportionately represented in non-college-bound tracks (Burris \& Wellner, 2005; Oakes, 1985; Sanders \& Holt, 1997; Zuniga et al., 2005) and that larger schools tend to proliferate the number of available tracks (Hallinan, 1994). Where there are more tracks, there is more potential for differential tracking. Moreover, differential tracking has been linked to a differential sense of student efficacy (Oakes, 1985), students' motivation and self-esteem (Hallinan, 1994), and a differential emphasis on higher order thinking skills in lower-track classes (Raudenbush et al., 1993).

School size has also been directly and negatively linked to standardized measures of student achievement (Abbott et al., 2002; Coladarci, 2006; C. Howley \& Bickel, 1999; Stewart, 2009; Weis et al., 2010); research shows that low SES and minority students differentially benefit from smaller school size (C. Howley, 1996; Lee \& Smith, 1997). Schools participating in the OSSI also showed the differential impact of school size on minority students. In these schools, the pre-existing achievement gap between OSSI and non-OSSI students steadily closed during the years of implementation. Finally, McMillen (2004) showed a similar, negative relationship between math achievement and school size among high school students.

Considered together, the results from these analyses support and corroborate the results reported in these analyses. For datasets one through three, school size was significantly and negatively related to minority AP Calculus 
production. In the case of the partially-specified model for dataset one, a one standard deviation increase in total enrollment resulted in a 0.158 standard deviation decrease in the production of minority AP calculus passing scores. The results for datasets two and three were in the same direction and of similar magnitudes indicating that school size was a reliable, negative predictor of minority AP Calculus production.

While these results are persistent and significant, they must be interpreted cautiously. Several researchers have noted important confounding effects that could serve to mask the impact of other variables nonrelated to school size (Coladarci, 2006; Weiss et al., 2010). Importantly, A. Howley and Howley (2006) noted that school size and pedagogy are often conflated, making the isolation of school size as an independent variable difficult. Differences in policies and practices are in fact not controlled for in these analyses. This effect is partially ameliorated by the size and geographic scope of the sample. Dataset one includes 133 schools enrolling over 220,000 students in seven distinct geographic regions. While policies and practices might be linked to school size in some cases, they are less likely to be systematically related to school size over the temporal and geographic range being investigated. The negative relationship between school size and minority AP Calculus production is therefore considered to be a reliable result within the limits of interpretation just discussed. The implications of this result are discussed later in this chapter. 
The strongest and most persistent result from these analyses is related to the relationship between overall success in non-Calculus AP exams for the total population and the success of minority students in AP Calculus. In every case, this variable emerged as the strongest and most significant predictor, with coefficients ranging between 0.417 and 0.686 for the fully-specified models for datasets one through three. For all of these cases, the coefficient estimates were significant to the $99 \%$ confidence level. A supplemental analysis showed that the rate of production for non-Calculus AP passing scores had a stronger impact on model fit than all other variables considered by a factor of nearly two. This variable in fact accounted for $15 \%$ of the variation in minority AP Calculus production. It is critical to note that these rates are normalized to the total size of the population being considered and not to the number of test takers in each group. These values are therefore production rates and not efficiency rates and are immune to the effects of selection that might artificially inflate traditional passing percentages. It is also critical to note that the overall success rates on AP exams did not include the number of Calculus exams passed. Because these two constructs were free to vary independently, the magnitude, significance, and persistence of this result represents an important finding.

Anecdotal evidence from Corbett High School and North Spokane High School cited earlier strongly suggested that as a culture of expectation and success began to take hold in a school, the rates of participation and success on various AP exams would increase. Several researchers included specific 
measures of "academic momentum" (Campbell, 2007) and "academic optimism" (W. Hoy et al., 2006) designed to assess the impact of school climate with respect to academic achievement. Both of these researchers demonstrated the significant and positive impact these constructs had on student achievement. Although academic momentum as constructed for these analyses did not appear as a significant predictor, the strength of the impact of total non-Calculus AP production strongly supported the "all boats rise on the same tide" phenomena described here. It was especially interesting to note that non-Calculus AP production for the total population was predictive of the production of minority AP Calculus passing scores. Theories of out group formation, stereotype threat, and oppositional culture all suggest that the success rate for minority groups might be expected to vary differently than the rates for the general population. In fact, this was not observed. The success of minority students in AP Calculus closely tracks with the rate of success of the total population on non-Calculus AP exams. The effect was significantly stronger than any other predictor, including proportion of economically disadvantaged or minority students enrolled. The precise mechanisms through which this variable operates are not clear from these analyses, but it is clear that whole-school culture with respect to AP success is a critical school-level predictor.

This final result was not anticipated in the hypotheses. Although several of the factors predicted to be important did rise to the level of significance, their collective impact was small in comparison. The degree to which a school 
produces a culture of AP success in general strongly predicts the success of minority of AP Calculus specifically. The impact of "academic emphasis" or "academic press" as a predictor of achievement has been established by the work of Hoy and others over several decades (Craig et al., 2001; Goddard et al., 2000; A. Hoy, 2012; W. Hoy et al., 2006; McGuigan \& Hoy, 2007; Picucci et al., 2002; Wagner \& DiPaola, 2011). This study reveals that academic emphasis as measured by the rate of whole-school success on non-Calculus AP exams is, by some wide margin, the most important predictor of minority success in AP Calculus.

Supplemental analyses described in the previous chapter more fully elucidated those school level factors which predict the production of nonCalculus AP passing scores. Interestingly, after controlling for the proportion of economically disadvantaged students, enrolled, school size again emerged as a significant and negative predictor of non-Calculus AP production. Per pupil teacher FTE also emerged as a significant and negative predictor of nonCalculus AP production, although, as noted earlier, important interaction effects make this result of dubious significance. School size was also shown to be significantly and negatively related to the total, time-averaged number of AP exams taken on a school level.

Subject to the limitations noted below, school size and school culture with respect to AP success appear to be strong and significant predictors of the rate of production of minority AP Calculus passing scores. Smaller schools in this 
sample more reliably produce passing scores on non-Calculus AP exams and minority students attending smaller schools more reliably pass AP Calculus exams. By a wide margin, the most important predictor of minority success on AP Calculus is the level of whole-school success on non-Calculus AP exams. The impact of school culture with respect to AP success appears to be definitive in these analyses. The implications of these two findings are discussed later in this chapter.

\section{Limitations}

The outcome variable itself imposed the most important limitation on this study. Approximately $40 \%$ of the 388 schools in the total sample reported nonzero minority success in AP Calculus, thereby significantly reducing the size of the available dataset. The inclusion of additional variables further reduced the size of the available sample. While 9 of the 10 districts were included in the largest dataset (dataset one), the number of longitudinal data points available was much smaller than the total number of data points initially collected. This limitation reduces the generalizability of results.

A second important limitation in these analyses deals with the non-normal distribution of the production of minority AP Calculus and production of total nonCalculus AP passing scores. Figures 6 and 7 show that both distributions are strongly and negatively skewed. While regression can proceed using nonnormally distributed outcome variables, this sort of skewed data can result in non-normally distributed residuals. The evaluation of $t$ tests is based on the 
assumption of normally-distributed residuals. Where this is not true, the significance of estimates of coefficients may be suspect (Regression with SPSS, n.d).

As regressions proceeded, residuals were computed and examined for degree of normal distribution. Figures 10 through 17 show histograms and Q-Q plots of unstandardized residuals for the partially-specified model for datasets one through three. For datasets two and three, the $Q-Q$ plots show a close agreement with the expected line for normal distribution. The $Q-Q$ plot for dataset one shows a reasonable agreement with normal distribution, but important exceptions should be noted. Specifically, four points with large-magnitude residuals vary widely from expected behavior. These points represent $3 \%$ of the total data points in dataset one and skew the overall distribution in the positive direction. The estimates of significance for the coefficients for this dataset are therefore slightly elevated. To ameliorate this problem, a transformation of APrelated data could be applied and a second round of analyses conducted and compared. These analyses, however, fall outside of the scope of the current investigation.

\section{Recommendations}

In the early 2000s, school size was at the center of the school-reform effort, particularly at the high school level. The Gates Foundation was at the forefront of this effort, funneling millions of dollars into various conversion, school-within-a-school, and new-start small schools across the country. Utilizing 
funds from the Gates foundation, 42 Oregon high schools initiated operation under a small school model between the 2003-2004 and 2009-2010 school years. Although results from these schools were promising in some instances, many school districts began closing or reconsolidating their small schools. In 2010, 22 schools in Oregon were still operating under a small schools model ("Schools and Results," n.d.). In 2008, Bill Gates signaled a shift in the educational emphasis of his Foundation, reporting that school size and school structure alone are necessary but not sufficient conditions for school improvement. School structure, Gates said, would continue to be a part of the Gates foundation's efforts because it "helps promote student success." However, teacher effectiveness and classroom effects would become the primary focus because "everything starts from that and must be built around it" (Gates, 2008, para. 21). This focus on teacher effects is reflected in President Obama's K-12 education priorities which seek advance initiatives that train, recruit and retain highly effective teachers and principals ("K-12 Education," n.d.).

Results from this investigation caution against a premature departure from school size as a reform strategy. Within the limitations discussed above, school size emerged as a significant and moderate predictor of AP-related outcome variables for the total student body and for minority students. Smaller schools administered more AP exams per 1,000 students, and students attending those smaller schools passed more Calculus and non-Calculus AP exams per 1,000 students. This effect emerged more consistently and had a stronger impact than 
the proportion of economically disadvantaged and minority students enrolled and per pupil teacher FTE. These data did not capture information with regards to concomitant variations in policy and practice. Therefore, the analyses presented here are not able to address the effect of school size independent of classroom level effects. However, they do support a broad but qualified conclusion with regards to school size: smaller high schools are generally more successful at creating success on AP exams for both the total population and for minority students. They also recommend a "stay the course" approach to those schools which are currently operating under a small school model. In Oregon between 2003 and 2010, schools in Newberg, Pendleton, Lebanon, and Portland which began operation with funding from OSSI reconsolidated or closed for a variety of reasons. This investigation strongly suggests that those that remain retain their small school focus. While perhaps not sufficient by itself, smaller school size provides a set of advantages which consistently manifest themselves with respect to AP success.

By a wide margin, the strongest and most significant finding of this investigation related to school culture. When the total student body produces more passing scores on non-Calculus AP exams per thousand students, minority students in those schools produce more passing scores on AP Calculus exams. Success on the AP English exam by the total student body, for instance, has a strong and significant impact on the success of Black and Hispanic students on the AP Calculus exam. Success on non-Calculus exams begets success on 
Calculus exams even more strongly than success on state math assessments. This effect persists across lines of ethnicity. Black and Hispanic students succeed in AP Calculus alongside and not separately from the total student body. The uneven distribution of success on Calculus and non-Calculus AP exams suggests that the combination of policies and practices that encourage this sort of whole-campus success on AP exams is rare. Where those policies and practices are in place, however, the results are strong and significant. This result holds even after controlling for proportion of economically disadvantaged and minority students enrolled, per pupil teacher FTE and per pupil building-level expenditures, and rates of success on state assessments of reading and math proficiency.

Earlier investigations of school factors that affect academic achievement (Goddard et al., 2000; W. Hoy et al., 2006) demonstrated the importance of academic emphasis within a school. Where "high academic goals are set for students, the learning environment is orderly and serious, students are motivated to work hard, and students respect academic achievement" then schoolaggregated academic success improves (W. Hoy et al., 2006, p. 427). Results from this study dramatically underscore Hoy's central findings. Within the constraints of these analyses, academic focus is definitive on a school level.

This result is particularly important because it emphasizes the importance of school culture in establishing and sustaining academic progress for all students, but especially for minority students. Banks (n.d.). Identified five 
dimensions of school culture which are impactful in terms of multicultural education: content integration, knowledge construction, equity pedagogy, prejudice reduction, and empowering school culture and school structure. These aspects are not directly operationalized in this investigation and are not captured in the datasets analyzed here. Nonetheless, they are likely to be on display in those schools which most effectively produce passing scores in AP Calculus posted by minority students. This investigation showed that just 14 schools (out of 361 schools with non-zero enrollment) produced half of the passing scores in AP Calculus by minority students; 12 of those schools are in one district. As described in the final section, a mixed-methods study of these 14 schools is strongly recommended.

\section{Opportunities for Future Research}

Data gaps represent the most important limitation in this investigation. Data collection was limited to those data points which were directly accessible through public, online data portals. The most critical refinement to this investigation therefore involves the inclusion of more data which, of necessity, would require the direct involvement of those already resource-constrained public school districts which generously provided requisite school codes. The reanalysis of a more extensive dataset may provide interesting insights into the interaction effects observed between per pupil teacher FTE and success on state assessments of reading and mathematics. Additionally, a more extensive dataset 
would allow for a more complete analysis of the effects of school expenditures on minority Calculus production.

As noted earlier, variations in school size are often conflated with variations in pedagogy, policy, and practice. Separating these effects would require the analysis of data describing teacher and classroom practices and school policies within the schools being examined. This sort of data would most effectively be collected with a qualitative, case study methodology. Since $50 \%$ of the total number of passing AP Calculus exams posted by minority students (in a time-averaged since) are accounted for by just 14 schools, a case study of these 14 schools would in fact capture most of the important variations. Additionally, 12 of these 14 schools are located in one school district and, counter to the general results with respect to school size, are larger than schools in the general population on average. It therefore seems possible that district-level policies are impacting between-school variations. These 12 schools represent an extremely attractive target for a qualitative or mixed-method analysis directly investigating school-aggregated and classroom-level practices.

While this investigation clearly points to the importance of academic emphasis it does not elucidate the mechanisms through which these constructs operate. School size appears to be an important covariate with AP success. In what ways does school size facilitate or accommodate a school-level emphasis on AP success? Academic momentum as constructed did not emerge as a reliable predictor even though non-Calculus AP success did. While it seems likely 
that data gaps contributed to this effect, these analyses were not able to clearly explain this mismatch. Is there a disconnect between AP success and success on state assessments that contributed to this? What might the cause of this disconnect be? Importantly, these data did not examine two of Hoy's three components of academic optimism: collective efficacy and faculty trust. In what ways do these components contribute to the observed connection between nonCalculus AP success and minority AP success?

Answers to many of these questions are likely obtainable through careful investigation of the 14 schools identified. There is a clear difference between these schools and the schools in the rest of the sample. It is possible-and perhaps even likely-that identifiable and scalable differences in policy and practice might emerge which together create a superior school-level culture of academic success. Because 12 of the 14 top producers of minority AP Calculus passing scores are from one district, there is a possibility that district-level effects could be important. Certain components of district-level leadership, for example, could prove to be definitive, along with district and school-level commitments to community connections, financial priorities, and specific, culturally-sensitive methods pedagogical practices. The lessons learned there may serve to widen and strengthen the STEM pipeline for Black and Hispanic students on a national level. 


\section{REFERENCES}

Abbott, M. L., Joireman, J., \& Stroh, H. R. (2002). The influence of district size, school size, and socioeconomic status on student achievement in Washington. Lynnwood, WA: Seattle Pacific University. (ERIC Document Reproduction Service No. ED470668)

Adelman, C. (1999). Answers in the toolbox: Academic intensity, attendance patterns, and bachelor's degree attainment. Washington, DC: U.S. Department of Education. (ERIC Document Reproduction Service No. ED431363)

Adelman, C. (2006). The toolbox revisited: Paths to degree completion from high school through college. Washington, DC: U.S. Department of Education. (ERIC Document Reproduction Service No. ED490195)

Adkins, R. (2012, July 9). America desperately needs more STEM students. Here's how to get them. Retrieved January 12, 2013, from http://www.forbes.com/sites/forbesleadershipforum/2012/07/09/americadesperately-needs-more-stem-students-heres-how-to-get-them/

Ainsworth-Darnell, J., \& Downey, D. (1998). Assessing the oppositional culture explanation for racial/ethnic differences in school performance. American Sociological Review, 63(4), 536-553.

Akiba, M., LeTendre, G., \& Scribner, J. (2009). Teacher quality, opportunity gap, and national achievement in 46 countries. Educational Researcher, 36(7), 369-387.

Allport, G. (1954). The nature of prejudice. New York, NY: Addison-Wesley.

American College Testing. (2005). College readiness begins in middle school. lowa City, IA: George Wimberly \& Richard Noeth.

Archer, L, DeWitt, J., Osborne, J., Dillon, J., Willis, B., \& Wong, B. (2012). Habitus: How families shape children's engagement and identification with science. American Educational Research Journal, 49(5), 881-908.

Archibald, D., Glutting, J., \& Qian, X. (2009). An analysis of the relative influence of grades, test scores, and race on track placement in a comprehensive high school. American Secondary Education, 37(2), 65-81. 
Aronson, J., Lustina, M., Good, C., \& Keough, K. (1999). When White men can't do math: Necessary and sufficient factors in stereotype threat. Journal of Experimental Psychology, 35, 29-46.

Attewell, P., Heil, S., \& Reisel, L. (2011). What is academic momentum? And does it matter? Educational Evaluation and Policy Analysis, 34(1), 27-44.

$\mathrm{Au}, \mathrm{W}$. (2005). Fresh out of school: Rap music's discursive battle with education. Journal of Negro Education, 74(3), 210-220.

Ayers, W., \& Klonsky, M. (2006). Chicago's renaissance 2010: The small school movement meets the ownership society. Phil Delta Kappan, 87(6), 453-457.

Banks, J. (n.d.). Multiculturalism's five dimensions. Retrieved from http://www. learner.org/workshops/socialstudies/pdf/session3/3.Multiculturalism.pdf

Belfield, C. (2011). Review of the comprehensive longitudinal evaluation of the Milwaukee Parental Choice Program: Summary of fourth year reports. Retrieved from http://nepc.colorado.edu/thinktank/review-milwaukeechoice-4.

Bifulco, R., \& Ladd, H. (2004). The impacts of charter schools on student achievement: Evidence from North Carolina. Retrieved from http://www.asu.edu/educ/epsl/EPRU/articles/EPRU-0412-76-OWI.pdf

Blascovich, J., Spencer, S., Quinn, D., \& Steele, C. (2001). African Americans and high blood pressure: The role of stereotype threat. Psychological Science, 12(3), 225-229.

Bonous-Hammarth, M. (2000). Pathways to success: Opportunities for science, mathematics, and engineering majors. The Journal of Negro Education, 69(1), 92-111.

Borman, G., \& Dowley, M. (2010). Schools and inequality: A multilevel analysis of Coleman's Equality of Education Opportunity data. Teacher's College Record, 112(5), 1201-1246.

Bourdieu, P. (2002). The forms of capital. In N. Biggart (Ed.), Economic sociology (pp. 46-58). Malden, MA: Blackwell Publishers Ltd. 
Bruno, J. (2002). The geographic distribution of teacher absenteeism in large urban school district settings: Implications for school reform efforts aimed at promoting equity and excellence in education. Education Policy Analysis Archives, 10(32). Retrieved from http://epaa.asu.edu.proxy.lib. pdx.edu/ ojs/article/view/311/437

Burris, C., \& Wellner, K. (2005). A special section on the achievement gap: Closing the achievement gap by de-tracking. Phi Delta Kappan, 86(8), 594-598.

Caldas, S., \& Bankston, C. (1997). Effect of school population socioeconomic status on individual academic achievement. The Journal of Educational Research, 90(5), 269-277.

Callahan, R. (2005). Tracking and high school English learners: Limiting opportunity to learn. American Educational Research Journal, 42(2), 305-328.

Campbell, D. (2007). An investigation into trends in Advanced Placement test taking in science and mathematics using student sub populations using a longitudinal growth model (Doctoral dissertation University of Southern California). Available from ProQuest Dissertations and Theses database. (UMI No. 3283693)

Card, D., \& Payne, A. (2002). School finance reform, the distribution of school spending, and the distribution of student test scores. Journal Public Economics, 82, 49-82.

CBS News. (May 23, 2012). Romney announces "unprecedented" education reforms [Video file]. Retrieved May 18, 2013, from http://www.cbsnews. com/video/watch/?id=7409494n

Chapa, J., \& De La Rosa, B. (2006). The problematic pipeline: Demographic trends and Latino participation in graduate science, technology, engineering, and mathematics programs. Journal of Hispanic Higher Education, 5(3), 203-221.

Chapman, C., Laird, J., \& Kewal Ramani, A. (2010). Trends in high school dropout and completion rates in the United States: 1972-2008 (NCES 2011-012). Washington, DC: National Center for Education Statistics, Institute of Education Sciences, U.S. Department of Education. Retrieved March 28, 2011, from http://nces.ed.gov/pubs2012/2012006.pdf 
Chubb, J. (1988). Why the current wave of school reform will fail. Public Interest, 90, 28-49.

Chubb, J., \& Moe, T. (1990). Politics, markets, and America's Schools. Washington, DC: The Brookings Institute.

Clotfelter, C., Ladd, H., \& Vigdor, L. (2007). How and why do teacher credentials matter for student achievement? (Working Paper No. 12828). Cambridge, MA: National Bureau of Economic Research.

Coladarci, T. (2006). School size, student achievement, and the "power rating" or poverty; substanative finding or statistical artifact? Educational Policy Analysis Archive, 14(28), 1-23.

Coleman, J. S., Campbell, E. Q., Hobson, C. J., McPartland, J., Mood, A. M., Weinfeld, F. D., \& York, R. L. (1966). Equality of educational opportunity: Summary report (vol. 2). Washington, DC: U.S. Department of Health, Education, and Welfare, Office of Education.

Coley, R. (1999). Opportunity offered-Opportunity taken: Course taking in American High Schools. ETS Policy Notes, 9(1), 132-153.

The College Board. (1999, October). Reaching the top: A report of the National Task Force on Minority High Achievement. New York, NY: Author.

The College Board. (2007). Student score distributions: AP exams [Data file]. Retrieved from http://research.collegeboard.org/programs/ap/data/archived/2007

The College Board. (2011). Student score distributions: AP exams [Data file]. Retrieved from http://research.collegeboard.org/programs/ap/data/ archived/2011

Condron, D., \& Roscigno, V. (2003). Disparities within: Unequal spending and achievement in an urban school district. Sociology of Education, 76(1), 18-36.

Conyers, J. (2012, November, 29). Republicans renew push for anti-minority STEM bill. Retrieved January 12, 2013, from http://thehill.com/blogs/congress-blog/education/

Counsel of Great City Schools (n.d.). Member districts. Retrieved from http://www.cgcs.org/domain/16. 
Cover, B., Jones, J., \& Watson, A. (2011, May). Science, technology engineering, and mathematics (STEM) occupations: A visual essay. Monthly Labor Review, Retrieved February 23, 2013, from http://www.bls.gov/opub/ mlr/2011/05/art1full.pdf

Craig, J., Butler, A., Cairo, L., Wood, C., Gilchrist, C., Holloway, J., Williams, S., \& Moats, S. (2001). A case study of six high performing schools in Tennessee. Charleston, WV: Appalachia Educational Laboratory at EDVANTIA.

Crane, J. (1996). Effects of home environment, SES, and maternal test scores on mathematics achievement. Journal of Educational Research, 89(5), 305-314.

Croizet, J., \& Claire, T. (1998). Extending the concept of stereotype threat to social class: The intellectual underperformance of students from low socioeconomic backgrounds. Personal and Social Psychology Bulletin, 24, 588-594.

Croninger, R. Rice, J., Rathbum, A., \& Nishio, M. (2007). Teacher qualifications and early learning: Effects of certification, degree, and experience on firstgrade achievement. Economics of Education Review, 26(3), 312-324.

Cubberley, E. P. (1919). Public education in the United States: A study and interpretation of American educational history. Boston, MA: Houghton Mifflin.

Darity, W., Castellino, D., Tyson, K., Cobb, C., \& McMillen, B. (2001). Increasing opportunity to learn via access to rigorous courses and programs: One strategy for closing the achievement gap for at-risk and ethnic minority students. Raleigh, NC: North Carolina Department of Public Instruction, Evaluation Section.

De Graaf, N. D., De Graaf, P. M., \& Kraaykamp, G. (2000). Parental cultural capital and educational attainment in the Netherlands: A refinement of the cultural captial perspective. Sociology of Education, 73(2), 92-111.

DiMaggio, P., \& Useem, M. (1998). Cultural democracy in a period of cultural expansion: The social composition of arts audiences in the United States. Social Problems, 26(2), 179-197.

Downey, D. (2008). Black/White differences in school performance: The oppositional culture explanation. Annual Review of Sociology, 34, 107-126. 
Dumais, S. (2002). Cultural capital, gender, and school success: The role of Habitus. Sociology of Education, 75(1), 44-68.

Employers for Education Excellence. (2010, November). Oregon Small Schools Initiative evaluation quantitative analysis 2004-2009. Portland, OR: Author.

Engberg, M., E., \& Wolniak, G. C. (2010). Examining the effects of high school context on postsecondary enrollment. Research in Higher Education, 51(2), 132-153.

Engler, J. (2012, June 15). STEM education is key to the U.S.'s economic future. U.S. News and World Report, Retrieved February 23, 2013, from http://www.usnews.com/opinion/articles/2012/06/15/stem-education-is-thekey-to-the-uss-economic-future

Evans, A., \& Rooney, B. (2008). Methods in psychological research. Los Angeles, CA: Sage.

Farkas, G., Lleras, C., \& Maczuga, S. (2002). Does oppositional culture exist in minority and poverty peer groups? American Sociological Review, 67(1), 148-155.

Fisher, E. (2007). Black student achievement and the cultural opposition model. The Journal of Negro Education, 74(3), 201-209.

Flores, A. (2007). Examining disparities in mathematics education: Achievement gap or opportunity gap? High School Journal, 91(1), 29-42.

Ford, D., Grantham, T., \& Whiting, G. (2008). Another look at the achievement gap: Learning from the experiences of gifted Black students. Urban Education, 43(2), 216-239.

Ford, H. (1926). Today and tomorrow: Garden City, NY: Double Day, Page \& Company.

Fordham, S., \& Ogbu, J. (1986). Black students' school success: Coping with the "burden of acting White." Urban Review, 18(3), 176-206.

Fries-Britt, S., Younger, T., \& Hall, T. (2010). Lessons learned from highachieving students of color in Physics. New Directions for Institutional Research, 148, 75-83. 
Gates, B. (2008, November 11). Bill Gates-A forum on education in America

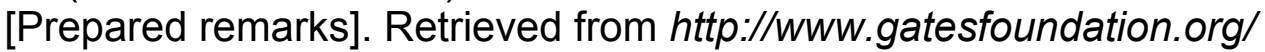
media-center/speeches/2008/11/bill-gates-forum-on-education-in-america

Gleason, P., Clark, M., Tutlte, C., Dwoyer, E., \& Silverberg, M. (2010). The evaluation of charter school impacts (Report No. NCEE 2010-4029). Washington, DC: National Center for Education Evaluation and Regional Assistance.

Goddard, R., Sweetland, S., \& Hoy, W. (2000). Academic emphasis of urban elementary schools and student achievement in reading and mathematics: A multilevel analysis. Education Administration Quarterly, 36(5), 683-702.

Goe, L. (2007). The link between teacher quality and student outcomes: A research synthesis. Washington, DC: National Comprehensive Center for Teacher Quality.

Goldthorpe, J. (2007). "Cultural capital": Come critical observations. Sociologica, $1(2), 1-23$.

Graham, E. (2013, April 25). "Nation at Risk" turns 30: Where did it take us? [Online news group]. Retrieved from http://neatoday.org/2013/04/25/anation-at-risk-turns-30-where-did-it-take-us/

Gross, S. (1993). Early mathematics performance and achievement: Results of a study within a large suburban school system. Journal of Negro Education, 62(3), 269-287.

Hall, D. (2013). Achievement gap or gap of opportunities. Urban Education, 48(2), 335-343.

Hallinan, M. (1994). School differences in tracking effects on achievement. Social Forces, 72(3), 799-820.

Hao, L., \& Bonstean-Bruns, M. (1998). Parent-child differences in educational expectations and the academic achievement of immigrant and native students. Sociology of Education, 71(3), 175-198.

Hara, S., \& Burke, D. (1998). Parent involvement: The key to improved student achievement. The School Community Journal, 8(2), 9-19. 
Hargrove, L, Godin, D., \& Dodd, B. (2008). College outcome comparisons by AP and non-AP high school experiences (Research Report No. 2008-3). Retrieved January 12, 2013, from: http://research.collegeboard.org/publications/content/2012/05/collegeoutcomes-comparisons-ap-and-non-ap-high-school-experiences

Harlow, C. W. (2003). Education and correctional populations (Report No. NCJ 195670). Washington, DC: United States Department of Justice.

Harris, A. (2006). I (don't) hate school: Revisiting the oppositional culture theory of Blacks' resistance to schooling. Social Forces, 85(2), 797-834.

Harris, A. (2007). Schooling behaviors or prior skills? A cautionary tale of omitted variable bias within oppositional culture theory. Sociology of Education, 80(2), 139-157.

Harris, D., \& Sass, T. (2008, March). Teacher quality, teacher training, and student achievement. Washington, DC: National Center for Analysis of Longitudinal Data in Education Research.

Harry, B., \& Anderson, M. (1996). Placement of African American males in special education programs: A critique of the process. Journal of Negro Education, 63(4), 602-619.

Haycock, K. (2002). Toward a fair distribution of teacher talent. Educational Leadership, 60(4), 11-15.

Herman, M. (2009). Black-White-other achievement gap: Testing theories of academic performance among multiracial and monoracial adolescents. Sociology of Education, 82(1), 20-46.

Hilton, T., \& Lee, V. (1988). Student interest and persistence in science: Changes in the educational pipeline in the last decade. Journal of Higher Education, 59(5), 510-526.

Howard, C. (2008). Who really cares? The disenfranchisement of African American males in prek-12 schools: A Critical Race Theory perspective. Teacher's College Record, 110(5), 954-985.

Howley, A., \& Howley, C. B. (2006). Small schools and the pressure to consolidate. Education Policy Analysis Archives, 14(10). Retrieved February 11, 2010 from http://epaa.asu.edu/v14n10/ 
Howley, C. (1996). Compounding disadvantage: The effects of school and district size on student achievement in West Virginia. Journal of Research in Rural Education, 12(1), 25-32.

Howley, C., \& Bickel R. (1999). The Matthew Project: National report. Randolph, VT: Rural Challenge Policy Program. (ERIC Document Reproduction Service No. ED 433 174)

Hoy, A. (2012). Academic optimism and teacher education. The Teacher Educator, 47(2), 91-100.

Hoy, W., Tarter, J., \& Bliss, J. (1990). Organizational climate and school health and effectiveness: A comparative analysis. Educational Administration Quarterly, 26(3), 260-279.

Hoy, W., Tarter, J., \& Hoy, A. (2006). Academic optimism: A force for student achievement. American Educational Research Journal, 43(3), 425-446.

Hrabowski, F. (2003). Raising minority achievement in science and math. Educational Leadership, 60(4), 44-48.

Ingersoll, R. (2002). Out-of-field teaching, educational inequality, and the organization of schools: An exploratory analysis (Document R-02-01). Seattle, WA: University of Washington, Center for the Study of Teaching and Policy.

Irving, M. A., \& Hudley, C. (2008). Cultural identification and academic achievement among African American males. Journal of Advanced Academics, 19, 676-698.

Jacobs, J. (2005). Twenty-five years of gender and ethnic differences in math and science career choices: What have we learned? New Directions for Child and Adolescent Development, 2005(110), 84-95.

Jeynes, W. (2005). A meta-analysis of the relation of parental involvement to urban elementary school student achievement. Urban Education, 40(5), 237-269.

K-12 education. (n.d.). Retrieved from http://www.whitehouse.gov/issues/ education $/ \mathrm{k}-12$

Kahle, J. (1998). Equitable systemic reform in science and mathematics: Assessing progress. Journal of Women and Minorities in Science and Engineering, 4(2-3), 91-112. 
Kaplan, D. (2009). Structural equation modeling: Foundations and extensions: Thousand Oaks, CA: Sage Publishing.

Kenyatta, C. (2012). From perception to practice: How teacher-student interactions affect African American male achievement. Journal of Urban Learning, Teaching, and Research, 8, 36-44.

Kao, G., \& Tienda, M. (1998). Educational aspirations among minority youth. American Journal of Education, 106(3), 349-384.

Kozol, J. (2005). The shame of the nation: The restoration of apartheid schooling in America. New York, NY: Crown Publishers.

Larson, P. (2012, July 18). President announces new plan to create STEM master teacher corps. Retrieved January 12, 2013, from http://www.whitehouse.gov/blog/2012/07/18/

Lee, V. E., \& Bryk, A. (1989). A multilevel analysis of the social distribution of high school achievement. Sociology of Education, 62(3), 172-192.

Lee, V. E., \& Smith, J. (1997). High school size: Which works best for whom? Educational Evaluation and Policy Analysis, 19(3), 205-227.

Levin, H. (1998). Educational vouchers: Effectiveness, choice, and cost. Journal of Policy Analysis and Management, 17(3), 373-392.

Lewis, J., Menzies, H., Najera, E., \& Page, R. (2009). Rethinking trends in minority participation in the sciences. Science Education, 9(3), 961-977.

Lewis, S., Simon, C., Renata, U., Horwitz, A., \& Casserly, M. (2010). A call for change: The social and educational factors contributing to the outcomes of Black males in urban schools. Retrieved from http://cgcs.schoolwires.net/cms/lib/DC00001581/Centricity/Domain/35/Pub lication\%20Docs/callforchange.pdf

Lewis, S., Simon, C., Renata, U., Horwitz, A., \& Casserly, M. (2011). Today's promise, tomorrow's future: The social and educational factors contributing to the outcomes of Hispanics in urban schools. Retrieved from http://www.cgcs.org/cms/lib/DC00001581/Centricity/domain/35/publication \%20docs/HispanicStudy2011.pdf

Lundy, G. (2003). The myths of oppositional culture. Journal of Black Studies, $33(4), 450-467$. 
Ma, X., \& Klinger, D. A. (2000). Hierarchical linear modelling of student and school effects on academic achievement. Canadian Journal of Education/Revue Canadienne de l'education, 41-55.

Mattern, K., Shaw, E., \& Xiong, X. (2009). The relationship between AP exam performance and college outcomes (Research Report No. 2009-4). Retrieved from http://research.collegeboard.org/publications/content/2012/05/relationshipbetween-ap-exam-performance-and-college-outcomes

McGuigan, L., \& Hoy, W. (2007). Principal leadership: Creating a culture of academic optimism to improve achievement for all students. Leadership and Policy in Schools, 45(5), 203-229.

McMillen, B. (2004). School size, achievement, and achievement gaps. Education Policy Analysis Archives, 12(58). Retrieved from http://epaa.asu.edu/epaa/v12n58/

Meyer, S. (1981). The five dollar day. Albany, NY: State University of New York Press.

Mickelson, R. (1990). The attitude-achievement paradox among Black adolescents. Sociology of Education, 63(1), 44-61.

Miron, G., \& Nelson, C. (2001). Student achievement in charter schools: What we know and why we know so little (Occasional Paper No. 41). New York, NY: Teacher's College, Columbia University, National Center for the Study of Privatization in Education.

Moore, J. (2006). A qualitative investigation of African-American males' career trajectory in engineering: Implications for teachers, school counselors, and parents. Teacher's College Record, 108(2), 246-266.

Muller, C. (1998). Gender differences in parental involvement and adolescents' mathematics achievement. Sociology of Education, 71(4), 336-356.

Murphy, D., \& Dodd, B. (2009). A Comparison of college performance of matched AP and non-AP students (Research Report No. 2009-6). Retrieved from http://research.collegeboard.org/publications/content/2012/05/comparisoncollege-performance-matched-ap-and-non-ap-student-groups

Museus, S., Palmer, R., \& Davis, R. (2011).Special issue: Racial and ethnic minority students' success in STEM education. AHSE Higher Education Report, 36(6), 1-140. 
My College Options \& STEMconnector. (2012). Where are the STEM students? What are their career interests? Where are the STEM jobs? 2012-2013 Executive summary. Retrieved from https://store.stemconnector.org/ Where-are-the-STEM-Students_p_15.html

National Science Board. (2004). Science and engineering indicators 2004 (vol.1, NSB 04-1; vol. 2, NSB 04-1A). Arlington, VA: National Science Foundation.

National Science Board. (2012). Science and Engineering Indicators 2012 (NSB 12-01). Arlington VA: National Science Foundation.

Oakes, J. (1985). Keeping Track: How Schools Structure Inequality. New Haven, CT: Yale University Press.

Oakes, J. (1990). Achievement and choice: Women and minority students in science and mathematics. Review of Research in Education, 16, 153-222.

Ogbu, J. (1982). Cultural discontinuities in schooling. Anthropology and Education Quarterly, 13(4), 290-307.

Ogbu, J. (1992). Understanding cultural diversity and learning. Educational Researcher, 21(8), 5-14.

Ogbu, J. (2004). Collective identity and the burden of "acting white" in Black history, community, and education. The Urban Review, 36(1), 1-35.

Oregon Department of Education. (2011a, October 21). Memorandum No. 0222011-2012-HB 3681. Salem, OR: Retrieved October 5, 2013, http://www. ode.state.or.us/news/announcements/announcement.aspx? $=7750$

Oregon Department of Education. (2011b, November 11). The Oregon STEM education initiative. Salem, OR: Retrieved January 12, 2013, from http://www.ode.state.or.us/search/results/?id=382.

Payne, K., \& Biddle, B. (1999). Poor school funding, child poverty, and mathematics achievement. Educational Researcher, 28(6), 4-13.

Perna, L. W. (2006). Studying college access and choice: A proposed conceptual model. In J. C. Smart (Ed.), Higher education: Handbook of theory and research (Vol. 21; pp. 99-157). Dordrecht, The Netherlands: Kluwer Academic Publishers. 
Picucci, A., Brownson, A., Kahlert, R., \& Sobel, A. (2002). Driven to succeed: High-performing, high-poverty, turnaround middle schools (Report No. UD 035-515). Austin, TX: University of Texas at Austin, The Charles A. Dana Center.

Raudenbush, S., Rowan, B., \& Cheong, Y. (1993). Higher order instructional goals: Class, teacher, and school influences. American Educational Research Journal, 30(3), 523-553.

Reardon, S. (2013, April 27). No rich child left behind. Opinionater. Retrieved June 2, 2013, from http://opinionator.blogs.nytimes.com/2013/04/27/norich-child-left-behind/

Reardon, S., Valentino, R., \& Shores, K. (2012). Patterns of literacy among U.S. students. The Future of Children, 22(2), 17-37.

Reese, W. J. (2005). America's public schools: From the common school the no child left behind. Baltimore, MD: John's Hopkins Press.

Regression with SPSS. (n.d.). Los Angeles, CA: University of California Los Angeles, Institute for Digital Research and Education. Retrieved from http://statistics.ats.ucla.edu/stat/spss/webbooks/reg/chapter1/spssreg1.ht $\mathrm{m}$

Riegle-Crumb, C. (2006). The path through math: Course sequences and academic performance at the intersection of race-ethnicity and gender. American Journal of Education, 79(4), 101-122.

Rodriguez, A. (2001). From gap gazing to promising cases: Moving toward equity in urban education reform. Journal of Research in Science Teaching, 38(10), 1115-1129.

Rose, H., \& Betts, J. (2004). The effect of high school courses on earnings. The Review of Economics and Statistics, 86(2), 497-513.

Rouse, C. (1998). Schools and student achievement: More evidence from the Milwaukee Parental Choice Program. Economic Policy Review, 4(1), 61-76.

Roza, M., Hill, P., Selafina, S., \& Speakman, S. (2004). How within-district spending inequities help some schools to fail. Brookings Papers on Educational Policy, 201-227. 
Rumberger, R., \& Larson, K., A. (1998). Toward explaining differences in educational achievement among Mexican American language-minority students. Sociology of Education. 71(1), 69-93.

Rumberger, R., \& Palardy, G. (2005). Does segregation still matter? The impact of student composition on academic achievement in high school. Teacher's College Record, 107(9), 1999-2045.

Russell, M., \& Atwater, M. (2004). Traveling the road to success: A discourse on persistence throughout the science pipeline with African-American students at a predominantly white institution. Journal of Research in Science Teaching, 42(6), 691-715.

Sadler, R., \& Tai, G. (2007).Advanced Placement exam scores as a predictor of performance in college Biology, Chemistry, and Physics courses. Science Educator, 16(1), 1-19.

Sanders, R., \& Holt, W. (1997, October 20). Still separate and unequal: Public education more than forty years after Brown. Motion Magazine. Retrieved January 12, 2013, from http://www.inmotionmagazine.com/forty.html

Sass, T. (2006).Charter schools and student achievement in Florida. Education Finance and Policy, 1(1), 91-122.

Schmader, T., \& Johns, M. (2003). Concerning evidence that stereotype threat reduces working memory capacity. Journal of Personality and Social Psychology, 85(3), 440-452.

Schools and results. (n.d.). Retrieved from http://www.e3smallschools.org/SRThe_Schools.html

Singer, J. (1998). Using SAS PROC MIXED to fit multilevel models, hierarchical, and individual growth models. Journal of Educational and Behavioral and Statistics, 24(4), 323-355.

Solorzano, D., \& Ornelas, A. (2002). A critical race analysis of Advanced Placement classes: A case of educational inequality. Journal of Latinos and Education, 11(4), 215-229.

Spencer, S., Steele, C., \& Quinn, D. (1998). Stereotype threat and women's math performance. Journal of Experimental Psychology, 35, 4-28.

Steele, C., \& Aronson, J. (1995). Stereotype threat and the intellectual test performance of African Americans. Journal of Personality and Social Psychology, 69(5), 797-811. 
STEM Jobs Act of 2013, S. 303-113 U.S.C. (2013). Retrieved from http://www.govtrack.us/congress/bills/113/s303

Stewart, L. (2009). Achievement differences between large and small schools in Texas. The Rural Educator, 30(2), 20-28.

Stone, J., Lynch, C., Sjomelig, M., \& Darley, J. (1999). Stereotype threat effects on Black and White athletic performance. Journal of Personality and Social Psychology, 77(6), 1213-1227.

Sullivan, A. (2001). Cultural capital and educational attainment. Sociology, 35(04), 893-912.

Tyson, W., Lee, R., Borman, K., \& Hanson, M. (2007). Science technology, engineering, and mathematics (STEM) pathways: High school science and math coursework and postsecondary degree attainment. Journal of Education for Students Placed at Risk, 121(3), 243-270.

United States Bureau of Labor Statistics. (2011). Unemployed and unemployment rates by educational attainment, sex, race, and Hispanic origin: 2000 to 2010. Retrieved September 3, 2012, from http://www.census.gov/ compendia/statab/2012/tables/12s0627.pdf

United States Census Bureau. (n.d.). U.S. Census Bureau projections show a slower growing, older, more diverse nation a half century from now [Press Release].Retrieved March 30, 2013, from https://www.census.gov/ newsroom/releases/archives/population/cb12-243.html

United States Census Bureau. (2012). Statistical abstract of the United States: 2012. Retrieved January 12, 2013, from http://www.census.gov/ prod/2011 pubs/12statab/pop.pdf

United States Department of Commerce. (2012, June). Public Education Finances: 2010. Retrieved January 12, 2013, from http://www2.census.gov/ govs/school/10f33pub.pdf

United States Department of Education, The National Commission on Education. (1983, April). A nation at risk: The imperative for educational reform. Retrieved May 1, 2013, from http://www.csus.edu/indiv///langd/ Nation_at_Risk.pdf

United States Office of Science and Technology Policy. (2012, February 13). Preparing a 21st century work force. Washington, DC: U.S. Government Printing Office. Retrieved January 12, 2013, from http://www. whitehouse.gov/ sites/default/files/microsites/ostp/fy2013rd_stem.pdf 
Wagner, C., \& DiPaola, M. (2011). Academic optimism of high school teachers: Its relationship to organizational citizenship behaviors. Journal of School Leadership, 21(6), 891-926.

Walliman, N. (2011). Research methods: The basics. London: Routledge.

Wang, M., \& Degol, J. (2013). Motivational pathways to STEM career choices: Using expectancy-value perspective to understand individual and gender differences in STEM fields. Developmental Review, 33, 304-340.

Washington State Commission on Asian Pacific American Affairs. (2009). Growing presence, emerging voices: Pacific Islanders and academic achievement in Washington. Retrieved from http://www.capaa.wa.gov/ documents/PacificlslanderAchievementGapReport.pdf

Wayne, A., \& Youngs, P. (2003). Teacher characteristics and student achievement gaps. Review of Educational Research, 73(1), 89-122.

Wei, T. (2012). Sticks, stones, words, and broken bones: New field and lab evidence on stereotype threat. Educational Evaluation and Policy Analysis, 34(4), 465-488.

Weiss, C. C., Carolan, B, V., \& Baker-Smith, E. C. (2010). Big school, small school: $(\operatorname{Re})$ Testing the assumptions about high school size, school engagement and mathematics achievement. Journal of Youth Adolescence, 39, 163-176.

Wolf, P. (2010). School vouchers in Washington, DC: Achievement impacts and their implications for social justice. Educational Research and Evaluation: An International Journal on Theory and Practice, 16(2), 131-150.

Zuniga, K., Olson, J., \& Winter, M. (2005). Science education for rural Latino/a students: Course placement and success in science. Journal of Research in Science Teaching, 42(4), 376-402. 


\section{APPENDIX}

\section{DATA SOURCES AND NOTES}




\section{Table A1}

Availability and Definitions of Enrollment Data

\begin{tabular}{|c|c|c|c|c|c|c|c|c|c|c|}
\hline & 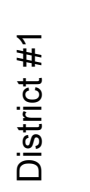 & 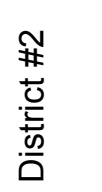 & 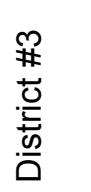 & 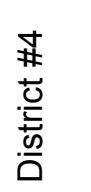 & 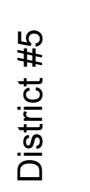 & 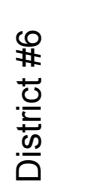 & 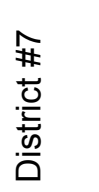 & 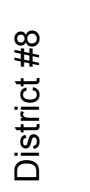 & 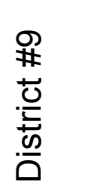 & 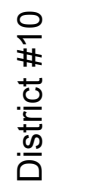 \\
\hline $\begin{array}{l}2007- \\
2008\end{array}$ & \# total & \# total & \# total & \# total & \# total & \# total & \# total & \# total & \# total & \# total \\
\hline $\begin{array}{l}2008- \\
2009\end{array}$ & \# total & \# total & \# total & \# total & \# total & \# total & \# total & \# total & \# total & \# total \\
\hline $\begin{array}{l}2009- \\
2010\end{array}$ & \# total & \# total & \# total & \# total & \# total & \# total & \# total & \# total & \# total & \# total \\
\hline $\begin{array}{l}2010- \\
2011\end{array}$ & \# total & \# total & \# total & \# total & \# total & \# total & \# total & \# total & \# total & \# total \\
\hline $\begin{array}{l}2011- \\
2012\end{array}$ & \# total & \# total & \# total & & \# total & \# total & \# total & \# total & \# total & \# total \\
\hline
\end{tabular}

\section{Table A2}

Notes for Data and Equivalencies, Enrollment

District

District \#1

District \#2

District \#3

District \#4

District \#5

District \#6

District \#7

District \#8

District \#9

District \#10

\section{Notes}

For "low income" student meets one of the following definitions: Free and Reduced Lunch (FRL) eligible, student is eligible for Transitional Aid to Families, or student is eligible for food stamps

"Economically disadvantaged" means a student who is eligible or whose sibling is eligible for $\mathrm{FRL}$, who is or whose guardians are eligible for public assistance, or whose guardians are eligible for Title 1 assistance.

Defined as "membership in special programs - FRL" according to NCES

Defined as FRL eligible or "Other economically disadvantaged"

Economically disadvantaged defined as FRL eligible

Economically disadvantaged defined as FRL eligible

Economically disadvantaged defined as FRL eligible

Economically disadvantaged defined as FRL eligible

Economically disadvantaged defined as FRL eligible 
Table A3

Availability and Definitions, State Assessments of Mathematics

\begin{tabular}{|c|c|c|c|c|c|c|c|c|c|c|}
\hline & 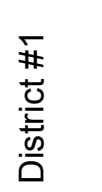 & $\begin{array}{l}\cong \\
\# \\
.00 \\
. \frac{0}{ \pm} \\
. \frac{0}{0}\end{array}$ & 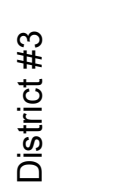 & $\begin{array}{l}\# \\
\# \\
+\frac{0}{2} \\
\frac{0}{0} \\
.0\end{array}$ & 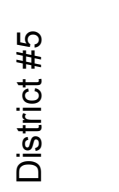 & 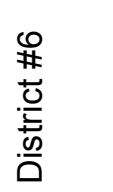 & $\begin{array}{l}\text { 告 } \\
+\frac{0}{0} \\
\frac{0}{ \pm} \\
\frac{0}{0}\end{array}$ & 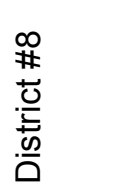 & 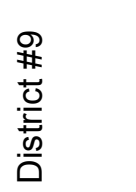 & 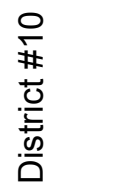 \\
\hline $\begin{array}{l}2007- \\
2008\end{array}$ & $\begin{array}{l}\% \text { prof. } \\
\text { or adv. } \\
\text { Grd } 10\end{array}$ & $\begin{array}{l}\% \text { at or } \\
\text { above } \\
\text { prof. }\end{array}$ & $\begin{array}{l}\% \\
\text { passing } \\
\text { scores on } \\
\text { EOC } \\
\text { tests* }\end{array}$ & $\begin{array}{l}\% \\
\text { passing, } \\
\text { Grd } 9\end{array}$ & $\begin{array}{l}\% \text { met } \\
\text { standard, } \\
\text { Grd } 10\end{array}$ & $\begin{array}{l}\% \\
\text { passing, } \\
\text { Grd } 9\end{array}$ & $\begin{array}{l}\% \text { met or } \\
\text { exceed } \\
\text { Grd } 10\end{array}$ & $\begin{array}{l}\text { \% prof. or } \\
\text { adv., } \\
\text { GRD 9-11 }\end{array}$ & $\begin{array}{l}\% \text { prof. or } \\
\text { adv., } \\
\text { GRD 9-11 }\end{array}$ & $\begin{array}{l}\text { \% prof. or } \\
\text { adv., } \\
\text { GRD 9-11 }\end{array}$ \\
\hline $\begin{array}{l}2008- \\
2009\end{array}$ & $\begin{array}{l}\% \text { prof. } \\
\text { or adv. } \\
\text { Grd } 10\end{array}$ & $\begin{array}{l}\% \text { at or } \\
\text { above } \\
\text { prof. }\end{array}$ & $\begin{array}{l}\% \\
\text { passing } \\
\text { scores on } \\
\text { EOC } \\
\text { tests* }\end{array}$ & $\begin{array}{l}\% \\
\text { passing, } \\
\text { Grd } 9\end{array}$ & $\begin{array}{l}\% \text { met } \\
\text { standard, } \\
\text { Grd } 10\end{array}$ & $\begin{array}{l}\% \\
\text { passing, } \\
\text { Grd } 9\end{array}$ & $\begin{array}{l}\% \text { met or } \\
\text { exceed } \\
\text { Grd } 10\end{array}$ & $\begin{array}{l}\text { \% prof. or } \\
\text { adv., Grd } \\
9-11\end{array}$ & $\begin{array}{l}\% \text { prof. or } \\
\text { adv., Grd } \\
9-11\end{array}$ & $\begin{array}{l}\text { \% prof. or } \\
\text { adv., Grd } \\
9-11\end{array}$ \\
\hline $\begin{array}{l}2009- \\
2010\end{array}$ & $\begin{array}{l}\% \text { prof. } \\
\text { or adv. } \\
\text { Grd } 10\end{array}$ & $\begin{array}{l}\% \text { at or } \\
\text { above } \\
\text { prof. }\end{array}$ & $\begin{array}{l}\% \\
\text { passing } \\
\text { scores on } \\
\text { EOC } \\
\text { tests* }\end{array}$ & $\begin{array}{l}\% \\
\text { passing, } \\
\text { Grd } 9\end{array}$ & $\begin{array}{l}\% \text { met } \\
\text { standard, } \\
\text { Grd } 10\end{array}$ & $\begin{array}{l}\% \\
\text { passing, } \\
\text { Grd } 9\end{array}$ & $\begin{array}{l}\% \text { met or } \\
\text { exceed } \\
\text { Grd } 10\end{array}$ & $\begin{array}{l}\% \text { prof. or } \\
\text { adv., Grd } \\
9-11\end{array}$ & $\begin{array}{l}\% \text { prof. or } \\
\text { adv., Grd } \\
9-11\end{array}$ & $\begin{array}{l}\text { \% prof. or } \\
\text { adv., Grd } \\
9-11\end{array}$ \\
\hline $\begin{array}{l}2010- \\
2011\end{array}$ & $\begin{array}{l}\% \text { prof. } \\
\text { or adv. } \\
\text { Grd } 10\end{array}$ & $\begin{array}{l}\% \text { at or } \\
\text { above } \\
\text { prof. }\end{array}$ & $\begin{array}{l}\% \\
\text { passing } \\
\text { scores on } \\
\text { EOC } \\
\text { tests* }\end{array}$ & $\begin{array}{l}\% \\
\text { passing, } \\
\text { Grd } 10\end{array}$ & $\begin{array}{l}\% \text { met } \\
\text { standard, } \\
\text { Grd } 10\end{array}$ & $\begin{array}{l}\% \\
\text { passing, } \\
\text { Grd } 10\end{array}$ & $\begin{array}{l}\% \text { met or } \\
\text { exceed } \\
\text { Grd } 11\end{array}$ & $\begin{array}{l}\text { \% prof. or } \\
\text { adv., Grd } \\
9-11\end{array}$ & $\begin{array}{l}\text { \% prof. or } \\
\text { adv., Grd } \\
9-11\end{array}$ & $\begin{array}{l}\text { \% prof. or } \\
\text { adv., Grd } \\
9-11\end{array}$ \\
\hline $\begin{array}{l}2011- \\
2012\end{array}$ & $\begin{array}{l}\% \text { prof. } \\
\text { or adv. } \\
\text { Grd } 10\end{array}$ & $\begin{array}{l}\% \text { at or } \\
\text { above } \\
\text { prof. }\end{array}$ & $\begin{array}{l}\% \\
\text { passing } \\
\text { scores on } \\
\text { EOC } \\
\text { tests* }\end{array}$ & & $\begin{array}{l}\% \text { met } \\
\text { standard, } \\
\text { Grd } 10\end{array}$ & & $\begin{array}{l}\% \text { met or } \\
\text { exceed } \\
\text { Grd } 11\end{array}$ & $\begin{array}{l}\text { \% prof. or } \\
\text { adv., Grd } \\
9-11\end{array}$ & $\begin{array}{l}\text { \% prof. or } \\
\text { adv., Grd } \\
9-11\end{array}$ & $\begin{array}{l}\% \text { prof. or } \\
\text { adv., Grd } \\
9-11\end{array}$ \\
\hline
\end{tabular}


Table A5

Availability and Definitions, State Assessments of Mathematics

\begin{tabular}{|c|c|c|c|c|c|c|c|c|c|c|}
\hline & 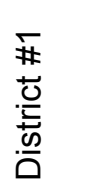 & $\begin{array}{l}\text { N } \\
\#+\frac{0}{0} \\
. \frac{0}{0} \\
.0\end{array}$ & 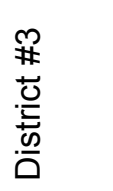 & 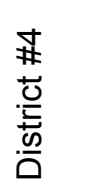 & 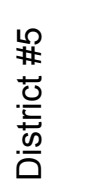 & 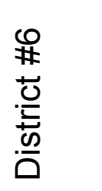 & 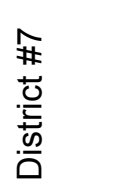 & 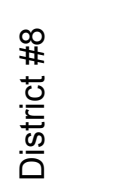 & 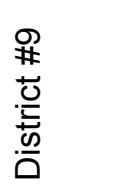 & 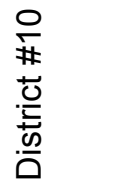 \\
\hline $\begin{array}{l}2007- \\
2008\end{array}$ & $\begin{array}{l}\% \text { prof. } \\
\text { or adv. } \\
\text { Grd } 10\end{array}$ & $\begin{array}{l}\% \text { at or } \\
\text { above } \\
\text { prof. }\end{array}$ & $\begin{array}{l}\% \text { pass } \\
\text { EOC } \\
\text { tests }\end{array}$ & $\begin{array}{l}\% \text { prof. } \\
\text { Grd } 9\end{array}$ & $\begin{array}{l}\% \text { met } \\
\text { grd } 10\end{array}$ & $\begin{array}{l}\% \text { prof. } \\
\text { grd } 9\end{array}$ & $\begin{array}{l}\% \text { met or } \\
\text { excd grd } \\
10\end{array}$ & $\begin{array}{l}\% \text { prof. } \\
\text { or adv., } \\
\text { grd } 9-11\end{array}$ & $\begin{array}{l}\text { \% prof. } \\
\text { or adv., } \\
\text { GRD 9- } \\
11\end{array}$ & $\begin{array}{l}\% \text { prof. } \\
\text { or adv., } \\
\text { grd } 9-11\end{array}$ \\
\hline $\begin{array}{l}2008- \\
2009\end{array}$ & $\begin{array}{l}\% \text { prof. } \\
\text { or adv. } \\
\text { Grd } 10\end{array}$ & $\begin{array}{l}\% \text { at or } \\
\text { above } \\
\text { prof. }\end{array}$ & $\begin{array}{l}\% \text { pass } \\
\text { EOC } \\
\text { tests }\end{array}$ & $\begin{array}{l}\% \text { prof. } \\
\text { Grd } 9\end{array}$ & $\begin{array}{l}\% \text { met } \\
\text { grd } 10\end{array}$ & $\begin{array}{l}\% \text { prof. } \\
\text { grd } 9\end{array}$ & $\begin{array}{l}\% \text { met or } \\
\text { excd grd } \\
10\end{array}$ & $\begin{array}{l}\% \text { prof. } \\
\text { or adv., } \\
\text { grd } 9-11\end{array}$ & $\begin{array}{l}\% \text { prof. } \\
\text { or adv., } \\
\text { Grd 9-11 }\end{array}$ & $\begin{array}{l}\% \text { prof. } \\
\text { or adv., } \\
\text { grd 9-11 }\end{array}$ \\
\hline $\begin{array}{l}2009- \\
2010\end{array}$ & $\begin{array}{l}\% \text { prof. } \\
\text { or adv. } \\
\text { Grd } 10\end{array}$ & $\begin{array}{l}\% \text { at or } \\
\text { above } \\
\text { prof. }\end{array}$ & $\begin{array}{l}\% \text { pass } \\
\text { EOC } \\
\text { tests }\end{array}$ & $\begin{array}{l}\% \text { prof. } \\
\text { Grd } 9\end{array}$ & $\begin{array}{l}\% \text { met } \\
\text { grd } 10\end{array}$ & $\begin{array}{l}\% \text { prof. } \\
\text { grd } 9\end{array}$ & $\begin{array}{l}\% \text { met or } \\
\text { excd grd } \\
10\end{array}$ & $\begin{array}{l}\% \text { prof. } \\
\text { or adv., } \\
\text { grd } 9-11\end{array}$ & $\begin{array}{l}\% \text { prof. } \\
\text { or adv., } \\
\text { Grd 9-11 }\end{array}$ & $\begin{array}{l}\% \text { prof. } \\
\text { or adv., } \\
\text { grd } 9-11\end{array}$ \\
\hline $\begin{array}{l}2010- \\
2011\end{array}$ & $\begin{array}{l}\% \text { prof. } \\
\text { or adv. } \\
\text { Grd } 10\end{array}$ & $\begin{array}{l}\% \text { at or } \\
\text { above } \\
\text { prof. }\end{array}$ & $\begin{array}{l}\% \text { pass } \\
\text { EOC } \\
\text { tests }\end{array}$ & $\begin{array}{l}\% \text { prof. } \\
\text { Grd } 9\end{array}$ & $\begin{array}{l}\% \text { met } \\
\text { grd } 10\end{array}$ & $\begin{array}{l}\% \text { prof. } \\
\text { grd } 9\end{array}$ & $\begin{array}{l}\% \text { met or } \\
\text { excd grd } \\
10\end{array}$ & $\begin{array}{l}\text { \% prof. } \\
\text { or adv., } \\
\text { grd } 9-11\end{array}$ & $\begin{array}{l}\text { \% prof. } \\
\text { or adv., } \\
\text { Grd 9-11 }\end{array}$ & $\begin{array}{l}\% \text { prof. } \\
\text { or adv., } \\
\text { grd } 9-11\end{array}$ \\
\hline $\begin{array}{l}2011- \\
2012\end{array}$ & $\begin{array}{l}\% \text { prof. } \\
\text { or adv. } \\
\text { Grd } 10\end{array}$ & $\begin{array}{l}\% \text { at or } \\
\text { above } \\
\text { prof. }\end{array}$ & $\begin{array}{l}\% \text { pass } \\
\text { EOC } \\
\text { tests }\end{array}$ & $\begin{array}{l}\% \text { prof. } \\
\text { Grd } 9\end{array}$ & $\begin{array}{l}\% \text { met } \\
\text { grd } 10\end{array}$ & $\begin{array}{l}\% \text { prof. } \\
\text { grd } 9\end{array}$ & $\begin{array}{l}\% \text { met or } \\
\text { excd grd } \\
10\end{array}$ & $\begin{array}{l}\% \text { prof. } \\
\text { or adv., } \\
\text { grd } 9-11\end{array}$ & $\begin{array}{l}\% \text { prof. } \\
\text { or adv., } \\
\text { Grd } 9-11\end{array}$ & $\begin{array}{l}\% \text { prof. } \\
\text { or adv., } \\
\text { grd } 9-11\end{array}$ \\
\hline
\end{tabular}


Table A6

Notes for Data and Equivalencies, State Assessments of Math and Reading

District

District \#1

District \#2

District \#3

District \#4

District \#5

District \#6

District \#7

District \#8

District \#9

District \#10 proficient or advanced, grade 10

\section{Notes}

Pass rate based on percent proficient or above on state test. Students must pass all 5 sections in order to receive a diploma. See:

Percentage of passing scores on required End of Course (EOC) tests. Tests given in multiple subjects. EOC results disaggregated by subgroup on state report cards but not by subject.

State assessment \% passing, Only grade 9 available for math for 2007-2008 through 2009-2010. Only grade 10 math available for 2010-2011. No math available for 2011-2012. Grades 9 and 10 available for reading. Only grade 9 used but grade 10 available in raw files for many schools. Grade 9 reading used because some schools only opened in 2012 with a freshman class. Using grade 9 expanded number of test scores available.

Percent met standard state assessment year of test (eg. 2008 in the 2007-2008 school year), grade 10, mathematics and English language arts. Certain charter schools did not report grade 10 results for ELA or math.

State assessment \% passing, Only grade 9 available for math for 2007-2008 through 2009-2010. Only grade 10 math available for 2010-2011. No math available for 2011-2012. Grades 9 and 10 available for reading. Only grade 9 used but grade 10 available in raw files for many schools. Grade 9 reading used because some schools only opened in 2012 with a freshman class. Using grade 9 expanded number of test scores available.

Percent met or exceeded standard, grade 10

State assessment percentage advanced and proficient in English-Language Arts (ELA-Reading), Algebra I and II, Integrated Math I and II, Geometry, Summative HS Math for Math. Total passing scores and total tests given in each test accumulated for grades 9-11. Passing percentage computed at number of tests passing divided by number of tests given for each subgroup. Note that number of tests passed for each test and each subgroup had to be computed from percentage passing rate in raw data file.

State assessment percentage advanced and proficient in English-Language Arts (ELA-Reading), Algebra I and II, Integrated Math I and II, Geometry, Summative HS Math for Math. Total passing scores and total tests given in each test accumulated for grades 9-11. Passing percentage computed at number of tests passing divided by number of tests given for each subgroup. Note that number of tests passed for each test and each subgroup had to be computed from percentage passing rate in raw data file.

State assessment percentage advanced and proficient in English-Language Arts (ELA-Reading), Algebra I and II, Integrated Math I and II, Geometry, Summative HS Math for Math. Total passing scores and total tests given in each test accumulated for grades 9-11. Passing percentage computed at number of tests passing divided by number of tests given for each subgroup. Note that number of tests passed for each test and each subgroup had to be computed from percentage passing rate in raw data file.

\section{Teacher data:}


Table A7

Availability and Definitions, Teacher FTE

\begin{tabular}{|c|c|c|c|c|c|c|c|c|c|c|}
\hline & 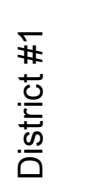 & 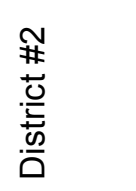 & 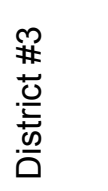 & 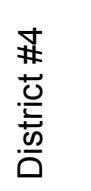 & 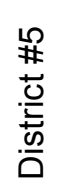 & 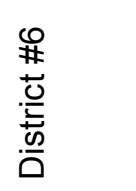 & 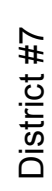 & 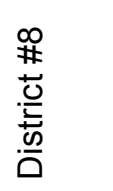 & 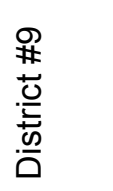 & 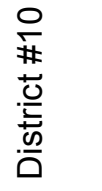 \\
\hline $\begin{array}{l}2007- \\
2008\end{array}$ & $\begin{array}{l}\text { Equiv. } \\
\text { Total } \\
\text { FTE }\end{array}$ & $\begin{array}{l}\text { Total \# of } \\
\text { FT } \\
\text { teachers }\end{array}$ & $\begin{array}{l}\text { Total \# } \\
\text { classroo } \\
\mathrm{m} \\
\text { teachers }\end{array}$ & $\begin{array}{l}\text { Equiv. } \\
\text { Total } \\
\text { FTE }\end{array}$ & $\begin{array}{l}\text { Total } \\
\text { FTE }\end{array}$ & $\begin{array}{l}\text { Equiv. } \\
\text { Total FTE }\end{array}$ & & $\begin{array}{l}\text { Total \# } \\
\text { teachers }\end{array}$ & $\begin{array}{l}\text { Total \# } \\
\text { teachers }\end{array}$ & $\begin{array}{l}\text { Total \# } \\
\text { teachers }\end{array}$ \\
\hline $\begin{array}{l}2008- \\
2009\end{array}$ & $\begin{array}{l}\text { Equiv. } \\
\text { Total } \\
\text { FTE }\end{array}$ & $\begin{array}{l}\text { Total \# of } \\
\text { FT } \\
\text { teachers }\end{array}$ & $\begin{array}{l}\text { Total \# } \\
\text { classroo } \\
\mathrm{m} \\
\text { teachers }\end{array}$ & $\begin{array}{l}\text { Equiv. } \\
\text { Total } \\
\text { FTE }\end{array}$ & $\begin{array}{l}\text { Total } \\
\text { FTE }\end{array}$ & $\begin{array}{l}\text { Equiv. } \\
\text { Total FTE }\end{array}$ & & $\begin{array}{l}\text { Total \# } \\
\text { teachers }\end{array}$ & $\begin{array}{l}\text { Total \# } \\
\text { teachers }\end{array}$ & $\begin{array}{l}\text { Total \# } \\
\text { teachers }\end{array}$ \\
\hline $\begin{array}{l}2009- \\
2010\end{array}$ & $\begin{array}{l}\text { Equiv. } \\
\text { Total } \\
\text { FTE }\end{array}$ & $\begin{array}{l}\text { Total \# of } \\
\text { FT } \\
\text { teachers }\end{array}$ & $\begin{array}{l}\text { Total \# } \\
\text { classroo } \\
\mathrm{m} \\
\text { teachers }\end{array}$ & $\begin{array}{l}\text { Equiv. } \\
\text { Total } \\
\text { FTE }\end{array}$ & $\begin{array}{l}\text { Total } \\
\text { FTE }\end{array}$ & $\begin{array}{l}\text { Equiv. } \\
\text { Total FTE }\end{array}$ & & $\begin{array}{l}\text { Total \# } \\
\text { teachers }\end{array}$ & $\begin{array}{l}\text { Total \# } \\
\text { teachers }\end{array}$ & $\begin{array}{l}\text { Total \# } \\
\text { teachers }\end{array}$ \\
\hline $\begin{array}{l}2010- \\
2011\end{array}$ & $\begin{array}{l}\text { Equiv. } \\
\text { Total } \\
\text { FTE }\end{array}$ & $\begin{array}{l}\text { Total \# of } \\
\text { FT } \\
\text { teachers }\end{array}$ & $\begin{array}{l}\text { Total \# } \\
\text { classroo } \\
\mathrm{m} \\
\text { teachers }\end{array}$ & $\begin{array}{l}\text { Equiv. } \\
\text { Total } \\
\text { FTE }\end{array}$ & $\begin{array}{l}\text { Total } \\
\text { FTE }\end{array}$ & $\begin{array}{l}\text { Equiv. } \\
\text { Total FTE }\end{array}$ & & $\begin{array}{l}\text { Total \# } \\
\text { teachers }\end{array}$ & $\begin{array}{l}\text { Total \# } \\
\text { teachers }\end{array}$ & $\begin{array}{l}\text { Total \# } \\
\text { teachers }\end{array}$ \\
\hline $\begin{array}{l}2011- \\
2012\end{array}$ & $\begin{array}{l}\text { Equiv. } \\
\text { Total } \\
\text { FTE }\end{array}$ & $\begin{array}{l}\text { Total \# of } \\
\text { FT } \\
\text { teachers }\end{array}$ & $\begin{array}{l}\text { Total \# } \\
\text { classroo } \\
\mathrm{m} \\
\text { teachers }\end{array}$ & & $\begin{array}{l}\text { Total } \\
\text { FTE }\end{array}$ & & & $\begin{array}{l}\text { Total \# } \\
\text { teachers }\end{array}$ & $\begin{array}{l}\text { Total \# } \\
\text { teachers }\end{array}$ & $\begin{array}{l}\text { Total \# } \\
\text { teachers }\end{array}$ \\
\hline
\end{tabular}


Table A7

Availability and Definitions, Percentage of Classes Taught by Highly Qualified Teachers

\begin{tabular}{|c|c|c|c|c|c|c|c|}
\hline 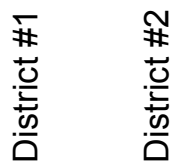 & 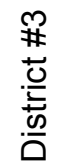 & 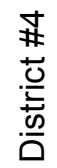 & 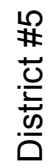 & 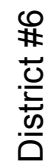 & 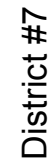 & 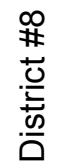 & 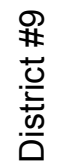 \\
\hline
\end{tabular}

2007- Pct. core Pct. core Pct.

$\begin{array}{llll}2008 & \text { aca. } & \text { classes by } & \text { classes } \\ \text { classes by } & \text { HQT } & \text { by HQT }\end{array}$ HQT

2008- Pct. core Pct. core Pct.

2009 aca. classes by classes

classes by HQT by HQT HQT

2009- Pct. core Pct. core Pct.

2010 aca. classes by classes classes by HQT by HQT HQT

2010- Pct. core Pct. core Pct.

2011 aca. classes by classes classes by HQT by HQT HQT

2011- Pct. core Pct. core Pct.

$2012 \quad$ aca. classes by classes classes by HQT by HQT HQT 
Table A7

Availability and Definitions, Teachers with at least a Bachelor's

\begin{tabular}{|c|c|c|c|c|c|c|c|c|c|}
\hline & 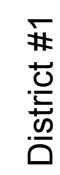 & 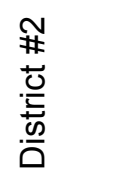 & 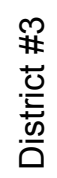 & 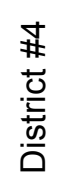 & 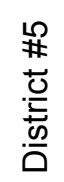 & 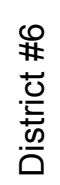 & 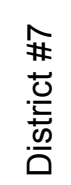 & 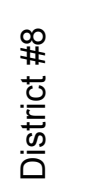 & 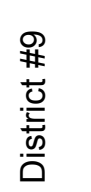 \\
\hline $\begin{array}{l}2007- \\
2008\end{array}$ & & $\begin{array}{l}\text { Total \# at } \\
\text { least } \\
\text { Bach }\end{array}$ & & & & & & \# total & \# total \\
\hline $\begin{array}{l}2008- \\
2009\end{array}$ & & $\begin{array}{l}\text { Total \# at } \\
\text { least } \\
\text { Bach }\end{array}$ & & & & & & \# total & $\#$ total \\
\hline $\begin{array}{l}2009- \\
2010\end{array}$ & & $\begin{array}{l}\text { Total \# at } \\
\text { least } \\
\text { Bach }\end{array}$ & & & & & & \# total & $\#$ total \\
\hline $\begin{array}{l}2010- \\
2011\end{array}$ & & $\begin{array}{l}\text { Total \# at } \\
\text { least } \\
\text { Bach }\end{array}$ & & & & & & \# total & $\#$ total \\
\hline $\begin{array}{l}2011- \\
2012\end{array}$ & & $\begin{array}{l}\text { Total \# at } \\
\text { least } \\
\text { Bach }\end{array}$ & & & & & & \# total & $\#$ total \\
\hline
\end{tabular}

Table A8

Availability and Definitions, Teachers with at least a Master's

\begin{tabular}{|c|c|c|c|c|c|c|c|}
\hline 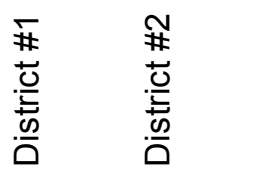 & 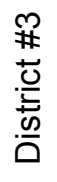 & 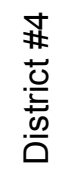 & 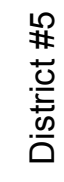 & 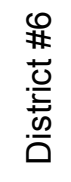 & $\begin{array}{l}\text { \# } \\
.0 \\
.0 \\
.00 \\
.0\end{array}$ & 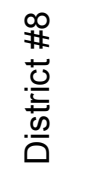 & 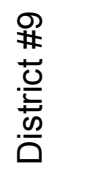 \\
\hline $\begin{array}{l}\text { Total \# at } \\
\text { least } \\
\text { Mast. }\end{array}$ & & & & & & $\#$ total & \# total \\
\hline $\begin{array}{l}\text { Total \# at } \\
\text { least } \\
\text { Mast. }\end{array}$ & & & & & & $\#$ total & $\#$ total \\
\hline $\begin{array}{l}\text { \#Total \# } \\
\text { at least } \\
\text { Mast. }\end{array}$ & & & & & & \# total & $\#$ total \\
\hline $\begin{array}{l}\text { Total \# at } \\
\text { least } \\
\text { Mast. }\end{array}$ & & & & & & $\#$ total & $\#$ total \\
\hline $\begin{array}{l}\text { Total \# at } \\
\text { least } \\
\text { Mast. }\end{array}$ & & & & & & $\#$ total & $\#$ total \\
\hline
\end{tabular}


Table A8

Availability and Definitions, Average Teacher's Salary

\begin{tabular}{|c|c|c|c|c|c|c|c|}
\hline 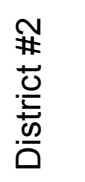 & $\begin{array}{l}m \\
\# \\
.0 \\
.0 \\
.00 \\
.00 \\
0.0\end{array}$ & 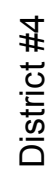 & 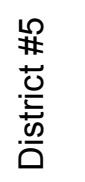 & 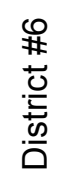 & 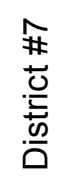 & 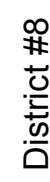 & 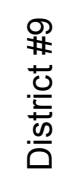 \\
\hline $\begin{array}{l}\text { Avg. } \\
\text { salary }\end{array}$ & & & $\begin{array}{l}\text { Avg. } \\
\text { salary }\end{array}$ & & & & \\
\hline $\begin{array}{l}\text { Avg. } \\
\text { salary }\end{array}$ & & & $\begin{array}{l}\text { Avg. } \\
\text { salary }\end{array}$ & & & & \\
\hline $\begin{array}{l}\text { Avg. } \\
\text { salary }\end{array}$ & & & $\begin{array}{l}\text { Avg. } \\
\text { salary }\end{array}$ & & & & \\
\hline $\begin{array}{l}\text { Avg. } \\
\text { salary }\end{array}$ & & & $\begin{array}{l}\text { Avg. } \\
\text { salary }\end{array}$ & & & & \\
\hline $\begin{array}{l}\text { Avg. } \\
\text { salary }\end{array}$ & & & $\begin{array}{l}\text { Avg. } \\
\text { salary }\end{array}$ & & & & \\
\hline
\end{tabular}

Table A9

Availability and Definitions, Average Teacher Experience

2007-

2008

2008-

2009

2009-

2010

2010

2011

2011-

2012

salary

salary 
Table A9

Availability and Definitions, Average Teacher Daily Attendance

\begin{tabular}{|c|c|c|c|c|c|c|c|}
\hline 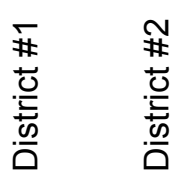 & 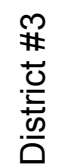 & 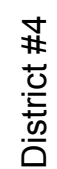 & 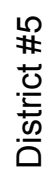 & $\begin{array}{l}0 \\
\# \\
.0 \\
.00 \\
.00 \\
.00\end{array}$ & 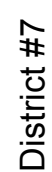 & 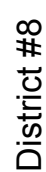 & 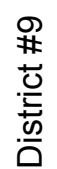 \\
\hline
\end{tabular}

2007-

2008

Pct. days

present

2008-

2009

2009-

2010

Pct. days

present

Pct. days

present

2010-

Pct. days

2011

present

2011-

Pct. days

2012

present

\section{Table A10}

Notes for Data and Equivalencies, Staff Information

District

District \#1

District \#2

District \#3

District \#4

District \#5

District \#6

District \#7

District \#8

District \#9

District \#10
Notes

Per pupil ratio and \% of teachers licensed in teaching assignment also available. Average teacher salaries available at district level.

FTE reported as total number of full time teachers, educational level reported as number with at least a bachelor's or master's degree. Report downloaded from website as year, school building data, teacher information

From state report cards, total number of classroom teachers and percentage of classes taught by HQT

FTE only from NCES site reported at total equivalent teacher FTE

Average actual salary (regular duties only) reported as average salary, average years of experience of teachers reported as average years experience.

FTE only from NCES site reported at total equivalent teacher FTE

Number of teachers with bachelor's and masters reported as total number, FTE reported as number of teachers (full time or not).

Number of teachers with bachelor's and masters reported as total number, FTE reported as number of teachers (full time or not).

Number of teachers with bachelor's and masters reported as total number, FTE reported as number of teachers (full time or not). 
Table A10

Availability and Definitions, Building-Level Administrative Expenditures

\begin{tabular}{|c|c|c|c|c|c|c|c|c|c|}
\hline & 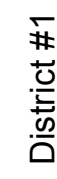 & 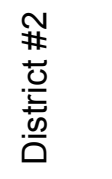 & 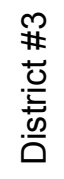 & 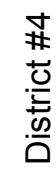 & 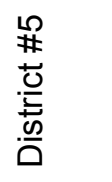 & 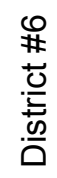 & 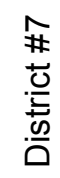 & 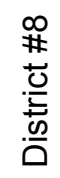 & 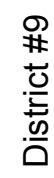 \\
\hline $\begin{array}{l}2007- \\
2008\end{array}$ & & $\begin{array}{l}\text { Total } \\
\text { expend. }\end{array}$ & & & $\begin{array}{l}\text { Total } \\
\text { expend. }\end{array}$ & & & & \\
\hline $\begin{array}{l}2008- \\
2009\end{array}$ & & $\begin{array}{l}\text { Total } \\
\text { expend. }\end{array}$ & & & $\begin{array}{l}\text { Total } \\
\text { expend. }\end{array}$ & & & & \\
\hline $\begin{array}{l}2009- \\
2010\end{array}$ & & $\begin{array}{l}\text { Total } \\
\text { expend. }\end{array}$ & & & $\begin{array}{l}\text { Total } \\
\text { expend. }\end{array}$ & & & & \\
\hline $\begin{array}{l}2010- \\
2011\end{array}$ & & $\begin{array}{l}\text { Total } \\
\text { expend. }\end{array}$ & & & $\begin{array}{l}\text { Total } \\
\text { expend. }\end{array}$ & & & & \\
\hline $\begin{array}{l}2011- \\
2012\end{array}$ & & $\begin{array}{l}\text { Total } \\
\text { expend. }\end{array}$ & & & $\begin{array}{l}\text { Total } \\
\text { expend }\end{array}$ & & & & \\
\hline
\end{tabular}

Table A11

Availability and Definitions, Building Operations Expenditures

\begin{tabular}{|c|c|c|c|c|c|c|c|}
\hline 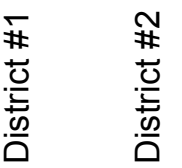 & 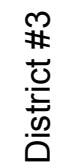 & 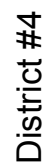 & 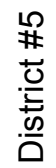 & 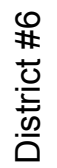 & 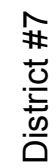 & 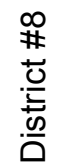 & 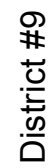 \\
\hline
\end{tabular}

2007-

2008

Total

expend.

2008-

2009

Total

expend.

2009-

2010

Total

expend.

2010 -

2011

2011 -

2012

Total

expend.

Total

expend. 
Table A12

Availability and Definitions, Support Staff Expenditures

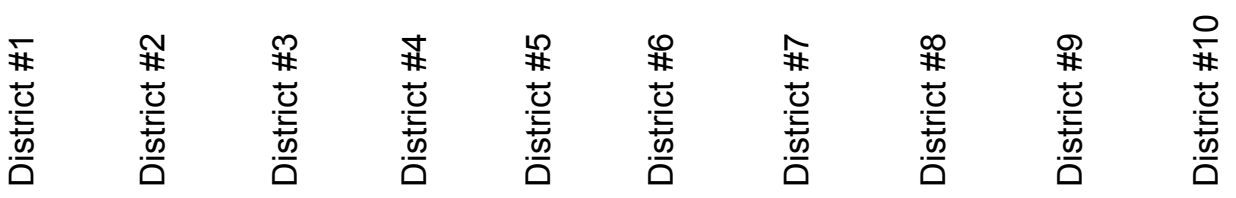

2007- Total

2008

expend.

2008- Total

2009 expend

2009- Total

2010 expend

2010- Total

2011 expend

2011- Total

2012

expend.

Table A13

Availability and Definitions, Pupil Support Expenditures

\begin{tabular}{|c|c|c|c|c|c|c|c|}
\hline 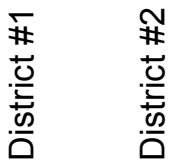 & 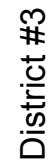 & 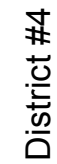 & 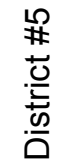 & 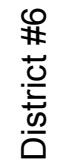 & 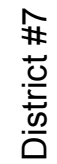 & 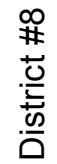 & 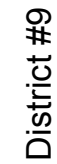 \\
\hline
\end{tabular}

2007-

2008

2008-

2009

2009-

2010

2010-

2011

2011-

2012

Total

expend.

Total

expend.

Total

expend.

Total

expend.

expend.

Total

expend.

Total

expend. 
Table A13

Availability and Definitions, Instructional Expenditures

\begin{tabular}{|c|c|c|c|c|c|c|c|c|c|}
\hline & 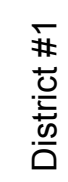 & 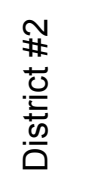 & 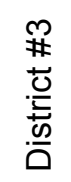 & 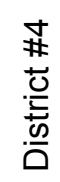 & 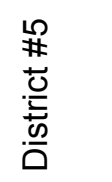 & 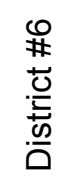 & 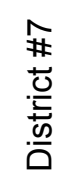 & 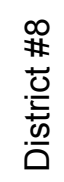 & 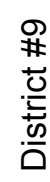 \\
\hline $\begin{array}{l}2007- \\
2008\end{array}$ & & $\begin{array}{l}\text { Total } \\
\text { expend. }\end{array}$ & & & $\begin{array}{l}\text { Total } \\
\text { expend. }\end{array}$ & & & & \\
\hline $\begin{array}{l}2008- \\
2009\end{array}$ & & $\begin{array}{l}\text { Total } \\
\text { expend. }\end{array}$ & & & $\begin{array}{l}\text { Total } \\
\text { expend. }\end{array}$ & & & & \\
\hline $\begin{array}{l}2009- \\
2010\end{array}$ & & $\begin{array}{l}\text { Total } \\
\text { expend. }\end{array}$ & & & $\begin{array}{l}\text { Total } \\
\text { expend. }\end{array}$ & & & & \\
\hline $\begin{array}{l}2010- \\
2011\end{array}$ & & $\begin{array}{l}\text { Total } \\
\text { expend. }\end{array}$ & & & $\begin{array}{l}\text { Total } \\
\text { expend. }\end{array}$ & & & & \\
\hline $\begin{array}{l}2011- \\
2012\end{array}$ & & $\begin{array}{l}\text { Total } \\
\text { expend. }\end{array}$ & & & $\begin{array}{l}\text { Total } \\
\text { expend. }\end{array}$ & & & & \\
\hline
\end{tabular}

Table A14

Availability and Definitions, Total Building-Level Expenditures

\begin{tabular}{|c|c|c|c|c|c|c|c|}
\hline 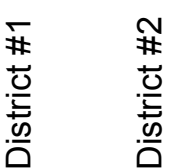 & 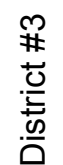 & 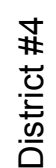 & 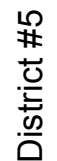 & 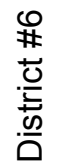 & 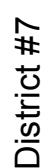 & 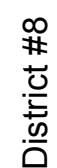 & 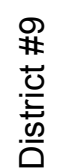 \\
\hline
\end{tabular}

2007-

2008

Total

expend.

Total

expend.

2008-

2009

2009-

2010

Total

expend.

Total

expend.

2010-

2011

2011-

2012

Total

expend.

Total

expend.

Total

expend.

Total

expend.

Total

Total

expend.

expend.

Data sources: 
Table A15

Notes for Data and Equivalencies, Expenditure Information

\section{District}

District \#1

District \#2

District \#3

District \#4

District \#5

District \#6

District \#7

District \#8

District \#9

District \#10

\section{Notes}

Total expenditures and per pupil expenditures available. For consistency purposes, use total expenditures in each category.

Total expenditures reported as total operating expenditures. Instructional expenditures reported as sum of instruction and instructional-related services. Administrative expenditures reported as school leadership. Pupil support reported as support services-student. 